\title{
Exact model categories, approximation theory, and cohomology of quasi-coherent sheaves
}

Jan Štovíček

\begin{abstract}
Our aim is to give a fairly complete account on the construction of compatible model structures on exact categories and symmetric monoidal exact categories, in some cases generalizing previously known results. We describe the close connection of this theory to approximation theory and cotorsion pairs. We also discuss the motivating applications with the emphasis on constructing monoidal model structures for the derived category of quasi-coherent sheaves of modules over a scheme.
\end{abstract}

2010 Mathematics Subject Classification. Primary: 55U35, 18G15; Secondary: 18E30, 18D10.

Keywords. Monoidal model category, exact category, derived category, cotorsion pair, weak factorization system, quasi-coherent sheaf.

\section{Contents}

1 Introduction

2 Quasi-coherent modules

3 Exact categories of Grothendieck type

4 Weak factorization systems

5 Complete cotorsion pairs

6 Exact and hereditary model categories $\quad 36$

7 Models for the derived category 48

8 Monoidal model categories from flat sheaves and vector bundles 56

\section{Introduction}

The aim of the present notes, partly based on the doctoral course given by the author at the University of Padova in March 2012, is twofold:

(1) to give a rather complete account on the construction of exact model structures and describe the link to cotorsion pairs and approximation theory; 
(2) to generalize the theory so that it applies to interesting recently studied classes of examples.

The parts of the paper related to (1) are mostly not new, except for the presentation and various improvements. However, there does not seem to be a suitable reference containing all the story and, as the adaptation to the algebraic setting sometimes requires small changes in the available definitions related to model categories, it seemed desirable to write up the construction at a reasonable level of details. Some results related to (2), on the other hand, are to our best knowledge original.

The concept of a model category [60, 41, 38, has existed for half a century. Despite being intensively studied by topologists, it has not attracted much attention in the theory of algebraic triangulated categories. There are probably two reasons for this development: The foundation of the theory of abelian and exact model categories has been only given a decade ago by Hovey [42] (see also [43] for a nice overview), and the "implementation details" for their construction are rather recent, see 223, 27, 28, 29, 30, 65, 71. In the meantime, a successful theory for algebraic triangulated categories has been developed, based on dg algebras and dg categories.

The exact model categories give in many respects a complementary approach to that of dg algebras, with different advantages and weaknesses, and it has a good potential for instance in Gorenstein homological algebra 39, homological algebra in the category of quasi-coherent sheaves [22, 23, 28, or in connection with recent developments regarding the Grothendieck duality [45, 56, 57, 58, 59, Interestingly, although model structures in connection with singularity categories are first explicitly mentioned in [6] by Becker, Murfet [56] and Neeman [57, 58, 59] still implicitly use parts of the theory which we are going to present.

While dg algebras provide perfect tools for constructing functors from single objects (say tilting or Koszul equivalences), the approach via models gives advantage in several theoretical questions. It may for example happen as in [45, 57] that a dg model for a given triangulated category is too complicated to understand, but the category itself has a rather easy description. There is, however, another important aspect - the model theoretic approach links the theory of triangulated categories to approximation theory [32, allowing deep insights on both sides. This is by no means to say that the dg and model techniques exclude each other-Keller in his seminal paper [47] in fact constructed two model structures for the derived category of a small dg category, and this point of view has been for example used to prove non-trivial results about triangulated torsion pairs in [68, §3.2].

Approximation theory is roughly speaking concerned with approximating general objects (modules, sheaves, complexes) by objects from special classes. Cofibrant and fibrant replacements in model categories are often exactly this kind of approximations. The central notion in that context is that of a cotorsion pair 64, whose significance has been recognized both in abstract module theory 32 and representation theory of finite dimensional algebras [4, Chapter 8]. The approach to construct approximations which we follow here started in [15], and the connection to model categories and Quillen's small object argument have been noticed in [42, 62] and in some form also in [7]. It was soon realized that similar results 
hold also for sheaf categories, for instance in [17, 18, 19.

It is fair to remark that there is an alternative approach to approximation theory, namely Bican's and El Bashir's proof of the Flat Cover Conjecture in [8] and its follow ups [16, 20, 40, 63, which does not seem to fit in our framework.

The first aim of ours, partly inspired by [21], but at a more advanced level, is to collect the essentials of the theory in one place together with a motivating and guiding example from [28, 23]: to construct for an arbitrary scheme $X$ a model structure for $\mathbf{D}(\mathrm{Q} \operatorname{coh}(X))$, the unbounded derived category of quasi-coherent sheaves, which is compatible with the tensor product. Of course, $\mathbf{D}(\mathrm{Q} \operatorname{coh}(X))$ may not be the category we wish to work with as we also have the subcategory of the derived category of all sheaves consisting of objects with quasi-coherent cohomology, but for many schemes the two categories are equivalent by [9, Corollary 5.5].

In order to achieve the goal, we also discuss in detail an equivalent description of $\mathrm{Q} \operatorname{coh}(X)$ as the category of certain modules over a representation of a poset in the category of rings. The description is due to Enochs and Estrada [17] and, although not very well suited for direct computations with coherent sheaves, it is excellent for theoretical questions regarding big sheaves. For example, it is a relatively straightforward task to prove that $\mathrm{Q} \operatorname{coh}(X)$ always is a Grothendieck category-compare to [69, B.2, p. 409]! This presentation is also quite accessible to the readers not acquainted with algebraic geometry.

As mentioned above, the other goal of the paper is to generalize the theory so that it is strong enough to apply to model structures in exact categories "appearing in the nature." Our motivation involves in particular an interpretation of recent results about singularity categories [45, 56, 57, 58, 59] and using models in conjunction with dg categories [68].

This program has been started by Saorín and the author in 65, 67] and it follows the spirit of [31. It is also, in a way, not a compulsory part for the reader, as it should be manageable to read the paper as if it were written only for, say, module categories instead of more general exact categories. Even in this restriction the presented results are relevant.

The main problem which we address here is a suitable axiomatics for exact categories which allows to use Quillen's small object argument and deconstructibility techniques to construct cotorsion pairs and model structures. The best suited concept so far seems to be an exact category of Grothendieck type defined in this text, although the theory is not optimal yet. The main problem is that we do not know whether the important Hill Lemma (Proposition 3.14) holds for these exact categories or in which way we should adjust the axioms to make it hold. As a consequence, some of our results including Proposition 3.19] Corollary 5.18 or Theorem 7.11 cannot be stated in as theoretically clean way as we would have wished. This is left as a possible direction for future research, where the promising directions include Enochs' filtration shortening techniques 20, or Lurie's colimit rearrangements from $[49, \S$ A.1.5] or 52 .

Acknowledgments. This research was supported by grant GA ČR P201/12/G028 from Czech Science Foundation. 


\section{Quasi-coherent modules}

In order to have classes of examples at hand, we start with describing the categories of quasi-coherent modules over schemes and diagrams of rings.

2.1. Grothendieck categories. Although the construction of model structures described later in this text has been motivated from the beginning by homological algebra in module and sheaf categories, several constructions work easily more abstractly for Grothendieck categories and, as we will discuss in Section 3 , even for nice enough exact categories. Thus we start with the definition and basic properties of Grothendieck categories.

Definition 2.1. 34 An abelian category $\mathcal{G}$ is called a Grothendieck category if

(Gr1) $\mathcal{G}$ has all small coproducts (equivalently: $\mathcal{G}$ is a cocomplete category).

$(\mathrm{Gr} 2) \mathcal{G}$ has exact direct limits. That is, given a direct system

$$
\left(0 \longrightarrow X_{j} \stackrel{i_{j}}{\longrightarrow} Y_{j} \stackrel{p_{j}}{\longrightarrow} Z_{j} \longrightarrow 0\right)_{j \in I}
$$

of short exact sequences, then the colimit diagram

$$
0 \longrightarrow \underset{j \in I}{\lim _{j}} X_{j} \longrightarrow \underset{j \in I}{\lim _{j}} Y_{j} \longrightarrow \underset{j \in I}{\lim _{j}} Z_{j} \longrightarrow 0
$$

is again a short exact sequence in $\mathcal{G}$. This is sometimes called the AB5 condition following an equivalent requirement in [34, p. 129].

$(\mathrm{Gr} 3) \mathcal{G}$ has a generator. That is, there is an object $G \in \mathcal{G}$ such that every $X \in \mathcal{G}$ admits an epimorphism $G^{(I)} \rightarrow X \rightarrow 0$. Here, $G^{(I)}$ stands for the coproduct $\coprod_{j \in I} G_{j}$ of copies $G_{j}$ of $G$.

An important property of a Grothendieck category is that it always has enough injective objects, which is very good from the point of view of homological algebra. This is in fact a good reason to consider infinitely generated modules or sheaves of infinitely generated modules: injective objects are often infinitely generated in any reasonable sense. We summarize the comment in a theorem:

Theorem 2.2. Let $\mathcal{G}$ be a Grothendieck category. Then each $X \in \mathcal{G}$ admits an injective envelope $X \rightarrow E(X)$. Moreover, $\mathcal{G}$ admits all small products (equivalently: it is complete) and has an injective cogenerator $C$. That is, $C$ is injective in $\mathcal{G}$ and each $X \in \mathcal{G}$ admits a monomorphism of the form $0 \rightarrow X \rightarrow C^{I}$.

Proof. The fact that every object $X \in \mathcal{G}$ admits a monomorphism $0 \rightarrow X \rightarrow E$ with $E$ injective was shown already in [34, Théorème 1.10.1]. The existence of injective envelopes and an injective cogenerator is proved in [55, Theorem 2.9] and [55, Corollary 2.11], respectively. The fact that $\mathcal{G}$ has products and many other properties of $\mathcal{G}$ are clear from the Popescu-Gabriel theorem, see e.g. 66, Theorem X.4.1]. 
2.2. Quasi-coherent modules over diagrams of rings. The simplest examples of Grothendieck categories are module categories $\mathcal{G}=\operatorname{Mod}-R$. In this section we construct more complicated examples, involving diagrams of rings and diagrams of modules over these rings. In fact, for suitable choices we obtain a category equivalent to the category of quasi-coherent sheaves over any given scheme. The presentation here is an adjusted version of [17, §2]. Since the discussion in [17, $\S 2]$ is rather brief and many details are omitted, we will also discuss in 2.3 the translation between quasi-coherent sheaves and the Grothendieck categories which we describe here.

Definition 2.3. Let $(I, \leq)$ be a partially ordered set. Then a representation $R$ of the poset $I$ in the category of rings is given by the following data:

(1) for every $i \in I$, we have a ring $R(i)$,

(2) for every $i \leq j$, we have a ring homomorphism $R_{j}^{i}: R(i) \rightarrow R(j)$, and

(3) we require that for every triple $i \leq j \leq k$ the morphism $R_{k}^{i}: R(i) \rightarrow R(k)$ coincides with the composition $R_{k}^{j} \circ R_{j}^{i}$, and also that $R_{i}^{i}=1_{R(i)}$.

Remark 2.4. If we view $I$ as a thin category in the usual way, then $R$ is none other than a covariant functor

$$
R: I \longrightarrow \text { Rings. }
$$

Remark 2.5. Although all of our examples and the geometrically minded motivation will involve only representations of posets in the category of commutative rings, non-commutative rings can be potentially useful too. For instance, one can consider sheaves of algebras of differential operators and ring representations coming from them. In any case, the commutativity is not necessary for the basic properties which we discuss in this section, so we do not include it in our definition.

Having defined representations of $I$ in the category of rings, we can define modules over such representations in a straightforward manner.

Definition 2.6. Let $R$ be a representation of a poset $I$ in the category of rings. A right $R$-module is

(1) a collection $(M(i))_{i \in I}$, where $M(i) \in \operatorname{Mod}-R(i)$ for each $i \in I$

(2) together with morphisms of the additive groups $M_{j}^{i}: M(i) \rightarrow M(j)$ for each $i \leq j$

(3) satisfying the compatibility conditions $M_{k}^{i}=M_{k}^{j} \circ M_{j}^{i}$ and $M_{i}^{i}=1_{M(i)}$ for every triple $i \leq j \leq k$, and such that

(4) the ring actions are respected in the following way: Given $x \in R(i)$ and $m \in M(i)$ for $i \in I$, then for any $j \geq i$ we have the equality

$$
M_{j}^{i}(m \cdot x)=M_{j}^{i}(m) \cdot R_{j}^{i}(x)
$$


All our modules in the rest of the text are going to be right modules unless explicitly stated otherwise, so we will omit usually the adjective "right". In order to obtain a category, it remains to define morphisms of $R$-modules. The definition is the obvious one.

Definition 2.7. Let $R$ be a representation of a poset $I$ in the category of rings and $M, N$ be $R$-modules. A morphism $f: M \rightarrow N$ is a collection of $(f(i): M(i) \rightarrow$ $N(i))_{i \in I}$, where $f(i)$ is a morphism of $R(i)$-modules for every $i \in I$, and the square

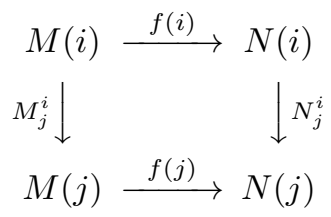

commutes for every $i<j$.

Let us denote the category of all $R$-modules by Mod- $R$. As we quickly observe:

Proposition 2.8. Let $(I, \leq)$ be a poset and $R$ a representation of $I$ in the category of rings. Then Mod- $R$ is a Grothendieck category. Moreover limits and colimits of diagrams of modules are computed component wise-we compute the corresponding (co)limit in $\operatorname{Mod}-R(i)$ for each $i \in I$ and connect these by the (co)limit morphisms.

Proof. Everything is very easy to check except for the existence of a generator in Mod- $R$. In fact, there is a generating set $\left\{P_{i} \mid i \in I\right\}$ of projective modules described as follows:

$$
P_{i}(j)= \begin{cases}R(j) & \text { if } j \geq i, \\ 0 & \text { otherwise }\end{cases}
$$

and the homomorphism $P_{i}(j) \rightarrow P_{i}(k)$ for $j \leq k$ either coincides with $R(j) \rightarrow R(k)$ if $i \leq j \leq k$ or vanishes otherwise.

One directly checks that there is a isomorphism

$$
\operatorname{Hom}_{R}\left(P_{i}, M\right) \cong M(i) \quad \text { for each } i \in I \text { and } M \in \operatorname{Mod}-R
$$

which assigns to every $f: P_{i} \rightarrow M$ the element $f(i)\left(1_{R(i)}\right) \in M(i)$. Moreover, the canonical homomorphism

$$
\coprod_{i \in I} P_{i}^{(M(i))} \longrightarrow M
$$

is surjective for every $M \in \operatorname{Mod}-R$, so $G=\coprod_{i \in I} P_{i}$ is a projective generator.

Although being valid Grothendieck categories, the categories Mod- $R$ as above are not the categories of our interest yet. In order to get a description of categories of quasi-coherent sheaves as promised, we must consider certain full subcategories instead. In order for this to work, we need an extra condition on $R$ : 
Definition 2.9. 17 Let $R$ be a representation of a poset $I$ in rings. We call $R$ a flat representation if for each pair $i<j$ in $I$, the ring homomorphism $R_{j}^{i}: R(i) \rightarrow R(j)$ gives $R(j)$ the structure of a flat left $R(i)$-module. That is,

$$
-\otimes_{R(i)} R(j): \operatorname{Mod}-R(i) \longrightarrow \operatorname{Mod}-R(j)
$$

is an exact functor.

As discussed later in $\$ 2.3$, the representations coming from structure sheaves of schemes always satisfy this condition. For such an $R$, we can single out the modules we are interested in:

Definition 2.10. Let $R$ be a flat representation of $I$ in rings. A module $M \in$ Mod- $R$ is called quasi-coherent if, for every $i<j$, the $R(j)$-module homomorphism

$$
\begin{aligned}
M(i) \otimes_{R(i)} R(j) & \longrightarrow M(j) \\
m \otimes x & \longmapsto M_{j}^{i}(m) \cdot x
\end{aligned}
$$

is an isomorphism.

Denote the full subcategory of Mod- $R$ formed by quasi-coherent $R$-modules by $\mathrm{Q} \operatorname{coh}(R)$.

Again, we obtain a Grothendieck category.

Theorem 2.11. Let $(I, \leq)$ be a poset and $R$ be a flat representation of $I$ in the category of rings. Then $\mathrm{Qcoh}(R)$ is a Grothendieck category. Moreover colimits of diagrams and limits of finite diagrams are computed component wise-that is, for each $i \in I$ separately.

Proof. Again, the main task is to prove that $\mathrm{Qcoh}(R)$ has a generator and the rest is rather easy, since taking colimits and kernels (hence also finite limits) commutes with the tensor products $-\otimes_{R(i)} R(j)$, where $i, j \in I$ and $i \leq j$. We omit the proof of the existence of a generator as it is rather technical, and refer to [17, Corollary 3.5] instead.

Remark 2.12. Every Grothendieck category has small products by Theorem 2.2. and so must have them $\mathrm{Q} \operatorname{coh}(R)$. However, these are typically not computed component wise and do not seem to be well understood. Since $\mathrm{Q} \operatorname{coh}(R)$ is a cocomplete category with a generator and the inclusion functor $\mathrm{Q} \operatorname{coh}(R) \rightarrow \operatorname{Mod}-R$ preserves small colimits, the inclusion $\mathrm{Q} \operatorname{coh}(R) \rightarrow \operatorname{Mod}-R$ has a right adjoint

$$
Q: \operatorname{Mod}-R \longrightarrow \operatorname{Qcoh}(R)
$$

by the special adjoint functor theorem [51, §5.8] (compare to [69, Lemma B.12]!) Following 69, we call such a $Q$ the coherator. Clearly, if $\left(M_{k}\right)_{k \in K}$ is a collection of quasi-coherent $R$-modules, the product in $\mathrm{Qcoh}(R)$ is computed as $Q\left(\prod M_{k}\right)$, where $\prod M_{k}$ stands for the (component wise) product in Mod- $R$. However, the abstract way of constructing $Q$ gives very little information on what $Q\left(\prod M_{k}\right)$ actually looks like. Some more information on this account is given in 69 , B.14 and B.15]. 
Before discussing a general construction in the next section, we exhibit particular examples of flat representations of posets of geometric origin and quasi-coherent modules over them.

Example 2.13. Consider the three element poset be given by the Hasse diagram

and a representation in the category of rings of the form

$$
R: \quad k[x] \stackrel{\subseteq}{\longrightarrow} k\left[x, x^{-1}\right] \stackrel{\supseteq}{\longleftarrow} k\left[x^{-1}\right],
$$

where $k$ is an arbitrary commutative ring. Clearly $R$ is a flat representation since the inclusions are localization morphisms.

For each $n \in \mathbb{Z}$, we have a quasi-coherent $R$-module

$$
\mathcal{O}(n): \quad k[x] \stackrel{\subseteq}{\longrightarrow} k\left[x, x^{-1}\right] \stackrel{x^{n} \cdot-}{\longleftarrow} k\left[x^{-1}\right] .
$$

One can easily check that $\mathcal{O}(m) \neq \mathcal{O}(n)$ whenever $m \neq n$, since by direct computation $\operatorname{Hom}_{R}(\mathcal{O}(m), \mathcal{O}(n))=0$ for $m>n$.

In fact, the category $\mathrm{Q} \operatorname{coh}(R)$ is equivalent to the category of quasi-coherent sheaves over $\mathbb{P}_{k}^{1}$, the projective line over $k$.

Example 2.14. Given a commutative ring $k$, let us now show a flat representation of a poset corresponding to the scheme $\mathbb{P}_{k}^{2}$, the projective plane over $k$. The Hasse diagram of the poset has the following shape:

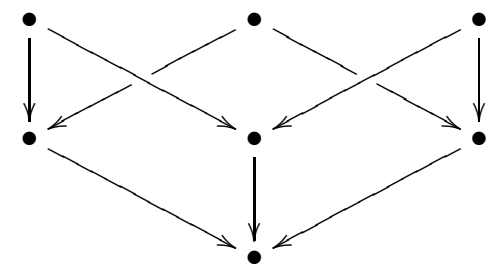

To describe the representation $R$ corresponding to $\mathbb{P}_{k}^{2}$, it is enough to define the ring homomorphisms corresponding to arrows in the Hasse diagram. Such a description is given in the following diagram, where all the rings are subrings of $k\left[x_{0}^{ \pm 1}, x_{1}^{ \pm 1}, x_{2}^{ \pm 1}\right]$, the ring of Laurent polynomials in three indeterminates over $k$, and all the ring homomorphisms are inclusions:

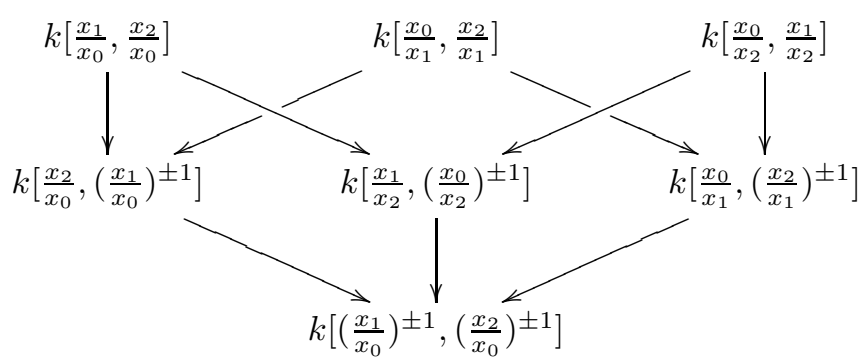


2.3. Realizing modules over schemes as modules over diagrams. The aim of this section is to make precise how the category of quasi-coherent sheaves of modules over a given scheme can be described in terms of quasi-coherent modules over a flat ring representation of a poset. Here we assume some familiarity with the basic notions in place: those of a scheme and of a quasi-coherent sheaf of modules over a scheme [37, 33. On the other hand, understanding this part is not necessary for understanding most of the text which follows, so the reader can skip it and continue with Section 3 .

Suppose that $\left(X, \mathcal{O}_{X}\right)$ is a scheme, that is a ringed space which is locally isomorphic to $\left(\operatorname{Spec} R, \mathcal{O}_{\operatorname{Spec} R}\right)$ for a commutative ring $R$. Given this data, we first construct a representation of a poset in the category of commutative rings.

Construction 2.15. Let $\mathcal{U}$ be a collection of open affine sets of $X$ satisfying the following two conditions:

(1) $\mathcal{U}$ covers $X$; that is $X=\bigcup \mathcal{U}$.

(2) Given $U, V \in \mathcal{U}$, then $U \cap V=\bigcup\{W \in \mathcal{U} \mid W \subseteq U \cap V\}$.

It is always a safe choice to take the collection of all affine open sets, but often much smaller sets $\mathcal{U}$ will do. For projective schemes for example, we can always choose $\mathcal{U}$ to be finite.

Now $\mathcal{U}$ is a poset with respect to inclusion and we put $I=\mathcal{U}^{\mathrm{op}}$, the opposite poset. Since $\mathcal{O}_{X}$ is a sheaf of commutative rings, we in particular have a functor

$$
\mathcal{U}^{\mathrm{op}} \longrightarrow \text { CommRings }
$$

which sends a pair $U \supseteq V$ of sets in $\mathcal{U}$ to the restriction $\operatorname{res}_{V}^{U}: \mathcal{O}_{X}(U) \rightarrow \mathcal{O}_{X}(V)$. By the very definition of $I$, this is the same as saying that we have a covariant functor $R: I \rightarrow$ CommRings such that, in the notation of Definition 2.3, we have $R(U)=\mathcal{O}(U)$ and $R_{V}^{U}=\operatorname{res}_{V}^{U}$.

A standard fact is that the representation of $I$ we get in this way is flat:

Lemma 2.16. Let $R$ be the representation of $I$ in the category of rings as in Construction 2.15. Then $R$ is flat.

Proof. This is proved for instance in [33, Proposition 14.3(1) and (4)]. Upon unraveling the definitions, the statements relies on the following fact from commutative algebra, [54, Theorem 7.1]: Given a homomorphism $\varphi: R \rightarrow S$ of commutative rings, then $S$ is flat over $R$ if and only if $S_{\mathfrak{q}}$ is flat over $R_{\varphi^{-1}(\mathfrak{q})}$ for every $\mathfrak{q} \in \operatorname{Spec} S$.

It is now easy to construct a functor from the category $\mathrm{Q} \operatorname{coh}(X)$ of quasicoherent sheaves of $\mathcal{O}_{X}$-modules to the category $\mathrm{Qcoh}(R)$ of quasi-coherent modules over $R$.

Construction 2.17. Let us adopt the notation from Construction 2.15, Given $M \in \mathrm{Q} \operatorname{coh}(X)$ and two affine open sets $U \supseteq V$, then canonically $M(U) \otimes_{\mathcal{O}_{X}(U)}$ 
$\mathcal{O}(V) \cong M(V)$-here we just apply [33, Remark 7.23 and Proposition 7.24(2)] to the open immersion

$$
\left(\operatorname{Spec} R(V), \mathcal{O}_{\operatorname{Spec} R(V)}\right) \cong\left(V, \mathcal{O}_{X \mid V}\right) \longrightarrow\left(U, \mathcal{O}_{X \mid U}\right) \cong\left(\operatorname{Spec} R(U), \mathcal{O}_{\operatorname{Spec} R(U)}\right)
$$

and the corresponding ring homomorphism $R_{V}^{U}: R(U) \rightarrow R(V)$.

Thus, viewing the sheaf $M$ as a contravariant functor from the poset of open sets of $X$ to $\mathrm{Ab}$, we may restrict the functor to $I=\mathcal{U}^{\text {op }}$. This way we assign to $M \in \mathrm{Q} \operatorname{coh}(X)$ an $R$-module $F(M)$ and $F(M)$ is quasi-coherent by the above discussion. This assignment is obviously functorial, so that we get an additive functor

$$
F: \operatorname{Qcoh}(X) \longrightarrow \mathrm{Q} \operatorname{coh}(R) \text {. }
$$

Seemingly, there is much more structure in $M \in \mathrm{Q} \operatorname{coh}(X)$ than in $F(M)$. While the former is a sheaf of modules over a possibly complicated topological space $X$, the latter is only a collection of modules satisfying a certain coherence condition. However, the fact that $M$ is quasi-coherent is itself very restrictive and we have the following crucial result; see [17, §2].

Theorem 2.18. The functor $F$ from Construction 2.17 (which depends on the choice of $\mathcal{U}$ in Construction 2.15) is an equivalence of categories.

Proof. Note that a quasi-coherent sheaf of modules $M \in \mathrm{Q} \operatorname{coh}(X)$ is determined up to a canonical isomorphism by its image under $F$. Indeed, this follows from conditions (1) and (2) in Construction 2.15, 33, Theorem 7.16(1)] and the sheaf axiom. Similarly a morphism $f: M \rightarrow N$ in $\operatorname{Qcoh}(X)$ is fully determined by $F(f)$. In particular $F$ is a faithful functor.

In order to prove that $F$ is dense, fix a module $A \in \mathrm{Q} \operatorname{coh}(R)$ and let us introduce some notation. Given an upper subset $L \subseteq I=\mathcal{U}^{\text {op }}$ with respect to the partial order $\leq$ on $I$, then $\bigcup L \subseteq X$ is an open subset of $X$, so that we can consider quasi-coherent sheaves over $\bigcup L$. We can also restrict the representation $R: I \rightarrow$ CommRings to the representation $L \rightarrow$ CommRings, which we denote by $R_{L}$. Clearly $R_{L}$ is a flat representation and Construction 2.17 provides us with a functor

$$
F_{L}: \operatorname{Qcoh}(\bigcup L) \rightarrow \operatorname{Qcoh}\left(R_{L}\right) .
$$

Finally, we have the restriction functor $\mathrm{Q} \operatorname{coh}(R) \rightarrow \mathrm{Q} \operatorname{coh}\left(R_{L}\right)$ and we will denote the image of $A$ under this functor by $\left.A\right|_{L}$.

Now we shall consider the collection $\Lambda$ of all upper subsets $L \subseteq I=\mathcal{U}^{\text {op }}$ such that there is a quasi-coherent sheaf of modules $M_{L} \in \mathrm{Q} \operatorname{coh}(\bigcup L)$ with $F_{L}\left(M_{L}\right)=$ $\left.A\right|_{L}$. As such $M_{L}$ is unique up to a canonical isomorphism, the collection $\Lambda$ is closed under unions of chains. Hence by Zorn's lemma there is an upper subset $L \subseteq I$ which belongs to $\Lambda$ and is maximal such with respect to inclusion. We claim that $L=I$. Suppose by way of contradiction that $L \subsetneq I$. Then there is $U \in \mathcal{U}^{\mathrm{op}}=I$ such that $U \nsubseteq \bigcup U L$ and we consider the unique quasi-coherent sheaf $\widetilde{A(U)} \in \mathrm{Q} \operatorname{coh}(U)$ whose global section module is $A(U)$. Now we invoke condition (2) of Construction 2.15 which, together with [33, Theorem 7.16(1)] and 
the sheaf axiom, allows us to construct a canonical isomorphism $\left.\left.\left(M_{L}\right)\right|_{V} \cong \widetilde{A(U)}\right|_{V}$ of sheaves over the open set $V=\bigcup L \cap U$. Thus we can glue $M_{L}$ and $\widetilde{A(U)}$ to a quasi-coherent sheaf over $\bigcup L \cup U$, showing that $L \cup\{W \in I \mid W \geq U$ in $I\}$ belongs to $\Lambda$, in contradiction to the choice of $L$. This proves the claim and the density of $F$.

The fact that $F$ is full is proved in a similar way. Given a morphism $g: A \rightarrow B$ in $\mathrm{Q} \operatorname{coh}(R)$, we denote by $\Lambda^{\prime}$ the collection of all upper subsets $L \subseteq I$ such that $\left.g\right|_{L}$ lifts to a morphism of sheaves of modules over the open subscheme $\bigcup L \subseteq X$. We ought to prove that $L=I$ and we again do so using Zorn's lemma.

\section{Exact categories of Grothendieck type}

In various contexts (see 65, 68 for example), it is useful to consider more general categories than Grothendieck categories. The rest of the text, however, is perfectly relevant when read as if it were written for Grothendieck categories or even for module categories. Thus, the reader who wishes to avoid the related technicalities may skip the section and read further from Section 4 .

3.1. Efficient exact categories. In order describe our object of interest, we recall some terminology. The central concept is that of an exact category, which is originally due to Quillen, but the common reference for a simple axiomatic description is [46. Appendix A] and an extensive treatment is given in [10.

An exact category is an additive category $\mathcal{E}$ together with a distinguished class of diagrams of the form

$$
0 \longrightarrow X \stackrel{i}{\longrightarrow} Y \stackrel{d}{\longrightarrow} Z \longrightarrow 0
$$

called conflations, satisfying certain axioms which make conflations behave similar to short exact sequences in an abelian category and allow to define Yoneda Ext groups with usual properties (see Section 5). Adopting the terminology from [46, the second map in a conflation (denoted by $i$ ) is called inflation, while the third map (denoted by $d$ ) is referred to as deflation.

Morally, an exact category is an extension closed subcategory of an abelian category, which is made precise in the following statement.

Proposition 3.1. [46, 10]

(1) Let $\mathcal{A}$ be an abelian category. Then $\mathcal{A}$ considered together with all short exact sequences as conflations is an exact category.

(2) Let $\mathcal{E}$ be an exact category and $\mathcal{E}^{\prime} \subseteq \mathcal{E}$ be an extension closed subcategory (i.e. if $0 \rightarrow X \rightarrow Y \rightarrow Z \rightarrow 0$ is a conflation and $X, Z \in \mathcal{E}^{\prime}$, then $Y \in \mathcal{E}^{\prime}$ ). Then $\mathcal{E}^{\prime}$, considered together with all conflations in $\mathcal{E}$ whose all terms belong to $\mathcal{E}^{\prime}$, is again an exact category.

(3) Every small exact category arises up to equivalence as an extension closed subcategory of an abelian category in the sense of (1) and (2). 
For the results presented later to work, we need to impose extra conditions on our exact categories. Long story short-we need to impose requirements on the exact category which make it sufficiently resemble a Grothendieck category. As the requirement that $\mathcal{E}$ be a cocomplete category seems too restrictive in practice, we need to specify first which direct limits we are interested in in connection with the analogue of the left exactness property (Definition $2.1(\mathrm{Gr} 2)$ ). In order to do so, the following definition is handy.

Definition 3.2. Let $\mathcal{C}$ be an arbitrary category, let $\lambda$ be an ordinal number, and let $\left(X_{\alpha}, f_{\alpha \beta}\right)_{\alpha<\beta<\lambda}$ be a direct system indexed by $\lambda$ in a category $\mathcal{C}$ :

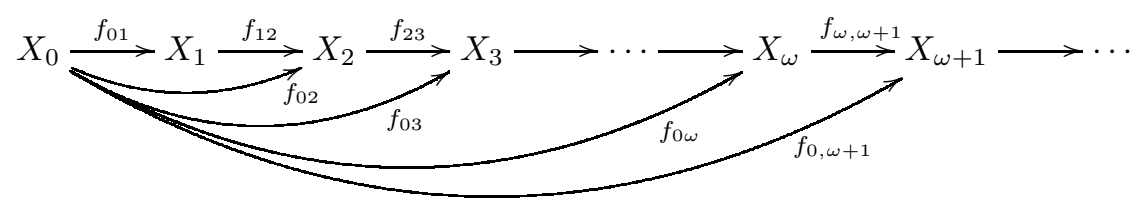

Such a system is called a $\lambda$-sequence if for each limit ordinal $\mu<\lambda$, the object $X_{\mu}$ together with the morphisms $f_{\alpha \mu}: X_{\alpha} \rightarrow X_{\mu}, \alpha<\mu$, is a colimit of the direct subsystem $\left(X_{\alpha}, f_{\alpha \beta}\right)_{\alpha<\beta<\mu}$. From now on, whenever we are going to depict a $\lambda$-sequence, we are going to draw only the morphism of the form $f_{\alpha, \alpha+1}$.

The composition of the $\lambda$-sequence is the colimit morphism

$$
X_{0} \longrightarrow \underset{\alpha<\lambda}{\lim } X_{\alpha}
$$

if it exists in $\mathcal{C}$.

Finally, if $\mathcal{I}$ is a class of morphisms of $\mathcal{C}$, then a transfinite compositions of morphisms of $\mathcal{I}$ are defined as the compositions of $\lambda$-sequences $\left(X_{\alpha}, f_{\alpha \beta}\right)_{\alpha<\beta<\lambda}$ with $f_{\alpha, \alpha+1} \in \mathcal{I}$ for every $\alpha+1<\lambda$.

For technical reasons for Quillen's small object argument in Section 4 , we need one more definition. The necessary set theoretical concepts can be found in [44].

Definition 3.3. If $\mathcal{C}$ is a category, $\kappa$ is a cardinal number and $\mathcal{D}$ is a class of morphisms of $\mathcal{C}$, then an object $X \in \mathcal{C}$ is called $\kappa$-small relative to $\mathcal{D}$ if, for every infinite regular cardinal $\lambda \geq \kappa$ and every $\lambda$-sequence

$$
E_{0} \longrightarrow E_{1} \longrightarrow E_{2} \longrightarrow \cdots \longrightarrow E_{\alpha} \longrightarrow E_{\alpha+1} \longrightarrow \cdots
$$

in $\mathcal{C}$ such that $f_{\alpha, \alpha+1}: E_{\alpha} \rightarrow E_{\alpha+1}$ is in $\mathcal{D}$ for all $\alpha+1<\lambda$, the canonical map of sets

$$
\underset{\alpha<\lambda}{\lim _{\alpha<\lambda}} \mathcal{C}\left(X, E_{\alpha}\right) \longrightarrow \mathcal{C}\left(X, \underset{\alpha<\lambda}{\lim _{\alpha<\lambda}} E_{\alpha}\right)
$$

is an isomorphism.

The object $X$ is called small relative to $\mathcal{D}$ if it is $\kappa$-small relative to $\mathcal{D}$ for some cardinal $\kappa$.

Then, modifying slightly the corresponding concept from 65, we state a first version of our specification which exact categories we are interested in. 
Definition 3.4. An exact category $\mathcal{E}$ is called efficient if

(Ef0) $\mathcal{E}$ is weakly idempotent complete. That is, every section $s: X \rightarrow Y$ in $\mathcal{E}$ has a cokernel or, equivalently by [10, Lemma 7.1], every retraction $r: Y \rightarrow Z$ in $\mathcal{E}$ has a kernel.

(Ef1) Arbitrary transfinite compositions of inflations exist and are themselves inflations.

(Ef2) Every object of $\mathcal{E}$ is small relative to the class of all inflations.

(Ef3) $\mathcal{E}$ admits a generator. That is, there is an object $G \in \mathcal{E}$ such that every $X \in \mathcal{E}$ admits a conflation $G^{(I)} \rightarrow X \rightarrow 0$.

Note that (Ef1) is a weak analogue of cocompleteness of $\mathcal{E}$ and left exactness of direct limits, while (Ef2) is a technical condition necessary for the small object argument in Corollary 4.10, Typical examples of efficient exact categories which we have in mind are Grothendieck categories (see Proposition 3.13 below) and various Frobenius exact categories used for the construction of algebraic triangulated categories (see for example [65, Theorem 4.2] or [68, Remark 2.15]). A systematic way of constructing more examples is given in $\$ 3.2$ using the notion of deconstructible classes.

Let us look closer at what we can say about efficient exact categories in general. An important fact whose proof is postponed to Corollary [5.5] is that the Yoneda Ext groups are always sets rather than proper classes. This is not a priori clear since efficient exact categories are practically never small. We also have infinite coproducts and these are exact.

Lemma 3.5. [65, Lemma 1.4] Let $\mathcal{E}$ be an exact category satisfying (Ef1) of Definition 3.4. Then the following hold:

(1) The category $\mathcal{E}$ has small coproducts.

(2) Small coproducts of conflations are conflations. lemma.

Condition (Ef0) is important because of the consequence stated in the next

Lemma 3.6. [10, Proposition 7.6] Let $\mathcal{E}$ be a weakly idempotent complete exact category and $f: X \rightarrow Y$ and $g: Y \rightarrow Z$ be a pair of composable morphisms. Then:

(1) If $g f$ is a inflation, then $f$ is a inflation.

(2) If $g f$ is a deflation, then $g$ is a deflation.

Beware, however, that unlike for Grothendieck categories an analog of Theorem 2.2 (enough injective objects) does not hold for efficient exact categories - see coming Example 3.12 We are going to give a remedy for that in the next subsection, at the cost of imposing another condition on $\mathcal{E}$. 
3.2. Exact categories of Grothendieck type. Here we are going to define exact categories of Grothendieck type which are even closer analogs of Grothendieck exact categories in that they always have enough injectives. Moreover, we shall describe a systematic procedure of producing these categories which seems to encompass all existing examples so far. The central concepts here are those of a filtration and deconstructibility.

Definition 3.7. Let $\mathcal{E}$ be an exact category and $\mathcal{S}$ be a class of objects in $\mathcal{E}$. By an $\mathcal{S}$-filtration we mean a $\lambda$-sequence

$$
0=X_{0} \stackrel{f_{01}}{\longrightarrow} X_{1} \stackrel{f_{12}}{\longrightarrow} X_{2} \stackrel{f_{23}}{\longrightarrow} X_{3} \longrightarrow \cdots \longrightarrow X_{\omega} \stackrel{f_{\omega, \omega+1}}{\longrightarrow} X_{\omega+1} \longrightarrow \cdots
$$

such that $X_{0}=0$ and for each $\alpha+1<\lambda$, the morphism $f_{\alpha, \alpha+1}$ is an inflation whose cokernel belongs to $\mathcal{S}$. That is, we have conflations

$$
0 \longrightarrow X_{\alpha} \stackrel{f_{\alpha, \alpha+1}}{\longrightarrow} X_{\alpha+1} \longrightarrow S_{\alpha} \longrightarrow 0
$$

with $S_{\alpha} \in \mathcal{S}$.

An object $X \in \mathcal{E}$ is called $\mathcal{S}$-filtered if $0 \rightarrow X$ is the composition (in the sense of Definition 3.2 ) of some $\mathcal{S}$-filtration. The class of all $\mathcal{S}$-filtered objects will be denoted by Filt $\mathcal{S}$.

Remark 3.8. Informally, an $\mathcal{S}$-filtration is just a transfinite extension of objects from $\mathcal{S}$. If, say, $\mathcal{E}=\operatorname{Mod}-R$ is the category of right $R$-modules for a $\operatorname{ring} R$ and we consider Mod- $R$ with the abelian exact structure (i.e. we take precisely all short exact sequences as conflations), then $X$ is $\mathcal{S}$-filtered if and only if there is a well ordered continuous chain $\left(X_{\alpha} \mid \alpha \leq \lambda\right)$ of submodules of $X$ such that $X_{0}=0, X_{\lambda}=X$ and $X_{\alpha+1} / X_{\alpha}$ is isomorphic to an object of $\mathcal{S}$ for each $\alpha+1 \leq \lambda$. This rather intuitive notion has been already successfully used for some time; see 32 and references there. As observed in 65, Definition 3.7 is a fairly wellbehaved generalization for efficient exact categories, although some care is due. For example, some properties of the generalized filtrations which would be clear for filtrations in module categories require a non-trivial proof for efficient exact categories- 65. Lemma 2.10] serves as an example.

A closely related concept is a deconstructible class in an exact category.

Definition 3.9. Let $\mathcal{E}$ be an exact category and $\mathcal{F} \subseteq \mathcal{E}$ be a class of objects. Then $\mathcal{F}$ is called deconstructible if there exists a set (not a proper class!) $\mathcal{S} \subseteq \mathcal{E}$ of objects such that $\mathcal{F}=$ Filt $\mathcal{S}$.

Let us summarize some basic properties of deconstructible classes, which we shall use freely in the sequel.

Lemma 3.10. Let $\mathcal{E}$ be an exact category satisfying (Ef1) of Definition 3.4 and let $\mathcal{F} \subseteq \mathcal{E}$ be a deconstructible class. Then any $\mathcal{F}$-filtered object of $\mathcal{E}$ belongs to $\mathcal{F}$. In particular, $\mathcal{F}$ is closed in $\mathcal{E}$ under taking coproducts and extensions. 
Proof. We refer to 65, Corollary 2.11] for the fact that $\mathcal{F}=$ Filt $\mathcal{F}$. While at the level of generality which we assume some work is necessary, the equality is an easy exercise in the special case where $\mathcal{E}=\operatorname{Mod}-R$ with the abelian exact structure. The fact that $\mathcal{F}$ is closed under extensions is then clear, and the closure under coproducts follows from the construction of coproducts via filtrations in the proof of [65, Lemma 1.4].

Now we can define exact categories of Grothendieck type.

Definition 3.11. An exact category $\mathcal{E}$ is said to be of Grothendieck type if

$(\mathrm{GT0}) \mathcal{E}$ is weakly idempotent complete.

(GT1) Arbitrary transfinite compositions of inflations exist and are inflations.

(GT2) Every object of $\mathcal{E}$ is small relative to the class of all inflations.

(GT3) $\mathcal{E}$ admits a generator.

(GT4) $\mathcal{E}$ is deconstructible in itself. That is, there exists a set of objects $\mathcal{S} \subseteq \mathcal{E}$ such that $\mathcal{E}=$ Filt $\mathcal{S}$

In other words, $\mathcal{E}$ is of Grothendieck type if $\mathcal{E}$ is efficient and satisfies (GT4). We recalled the former axioms for the reader's convenience. As already mentioned, the key consequence of (GT4) is that $\mathcal{E}$ has enough injective objects. We postpone the proof of the fact to Corollary 5.9 when we will have developed the necessary theory. Here we rather focus on how exact categories of Grothendieck type occur, but first we show a non-example demonstrating that (GT4) is indeed necessary.

Example 3.12. Let $\mathcal{D} \subseteq \mathrm{Ab}$ be the category of all flat Mittag-Leffler abelian groups in the sense of 61]. These groups are characterized by the property that every countable subgroup is free; see [5. Proposition 7]. It is not difficult to prove that $\mathcal{D}$ is closed under retracts, any $\mathcal{D}$-filtered object belongs to $\mathcal{D}$, and that $\mathbb{Z}$ is a generator for $\mathcal{D}$. Hence $\mathcal{D}$ is an efficient exact category. On the other hand, $\mathcal{D}$ cannot be deconstructible in itself by [23, Corollary 7.3]. Even worse, the only injective object of $\mathcal{D}$ is the zero object, so $\mathcal{D}$ does not have enough injectives. To see that, observe that by [23, Theorem 5.5] $X \in \mathcal{D}$ is injective in $\mathcal{D}$ if and only if $X$ is a so-called cotorsion group. However, the only flat Mittag-Leffler cotorsion group is by [14, Corollary V.2.10(ii)] the zero group.

Turning back to examples, all Grothendieck categories are exact categories of Grothendieck type. In order to show that, we recall a few standard facts. One can define a subobject of an object $X$ in an abelian category $\mathcal{G}$ as an equivalence class of monomorphisms $Y \rightarrow X, 66$, §IV.2]. We shall as usual write $Y \subseteq X$ in such a case. If $\mathcal{G}$ is a Grothendieck category, then the subobjects of a given object form a modular upper continuous complete lattice $(\operatorname{Subobj}(X),+, \cap), 66$, Propositions IV.5.3 and V.1.1 (c)]. Recall a complete lattice $(\mathcal{L}, \vee, \wedge)$ is upper continuous (cf. [66, §III.5]) if $\left(\bigvee_{d \in D} d\right) \wedge a=\bigvee_{d \in D}(d \wedge a)$ whenever $a \in \mathcal{L}$ and $D \subseteq \mathcal{L}$ is a directed subset. 
Proposition 3.13. Let $\mathcal{G}$ be a Grothendieck category considered with the abelian exact structure. Then $\mathcal{G}$ is of Grothendieck type as an exact category.

Proof. Condition (GT0) is clearly true for every abelian category. Since $\mathcal{G}$ is cocomplete and has exact direct limits, (GT1) holds. The existence of a generator (GT3) is a part of the definition of a Grothendieck category. Regarding the smallness assumption (GT2), it is a well-known fact that any object $A \in \mathcal{G}$ is small relative to the class all morphisms. Indeed, the Popescu-Gabriel theorem 66, Theorem X.4.1] guarantees that for any fixed $A \in \mathcal{G}$, there is a cardinal number $\kappa$ such that

(1) $\mathcal{G}$ identifies with a full subcategory of $\operatorname{Mod}-R$, the category of right modules for some ring $R$, such that $\mathcal{G}$ is closed in Mod- $R$ under taking colimits of $\lambda$-sequences for every infinite regular cardinal $\lambda \geq \kappa$, and

(2) under this identification $A$ can be presented, as an $R$-module, by fewer than $\kappa$ generators and relations, so that the functor

$$
\operatorname{Hom}_{R}(A,-): \text { Mod- } R \longrightarrow \mathrm{Ab}
$$

commutes with colimits of $\lambda$-sequences for every infinite regular $\lambda \geq \kappa$.

Finally, let $\mathcal{S}$ be a representative set of quotients $\{G / Y \mid Y \subseteq G\}$, where $G$ is a generator of $\mathcal{G}$. Given any object $X \in \mathcal{G}$ and a fixed epimorphism $p: G^{(I)} \rightarrow X$, we shall construct an $\mathcal{S}$-filtration of $X$. This will prove (GT4). To this end, we can without loss of generality assume that $I=\lambda$ is an ordinal number and define $X_{\alpha} \subseteq X$ for each $\alpha \leq \lambda$ as the image of restriction of $p$ to $G^{(\alpha)}$. It is not difficult to convince oneself that $\left(X_{\alpha}\right)_{\alpha \leq \lambda}$ is (by slightly abusing the notation) an $\mathcal{S}$-filtration of $X$.

Our next goal is to prove that every deconstructible class of a Grothendieck category, viewed as a full subcategory, is naturally an exact category of Grothendieck type. In order to do so, we need a technical tool: the generalized Hill Lemma. Here we take a slightly restricted version of the result from [67, where also references to other versions and evolution stages of the Hill Lemma can be found.

Proposition 3.14. Let $\mathcal{G}$ be a Grothendieck category and $\mathcal{S}$ be a set of objects. Then there exists an infinite regular cardinal $\kappa$ with the following property: For every $X \in \mathcal{G}$, which is the union of an $\mathcal{S}$-filtration

$$
0=X_{0} \subseteq X_{1} \subseteq X_{2} \subseteq \cdots \subseteq X_{\alpha} \subseteq X_{\alpha+1} \subseteq \cdots \subseteq X_{\sigma}=X
$$

for some ordinal $\sigma$, there is a complete sublattice $\mathcal{L}$ of the power $\operatorname{set}(\mathcal{P}(\sigma), \cup, \cap)$ of $\sigma$ and a map

$$
\ell: \mathcal{L} \longrightarrow \operatorname{Subobj}(X)
$$

which assigns to each $S \in \mathcal{L}$ a subobject $\ell(S)$ of $X$, such that the following hold:

(H1) For each $\alpha \leq \sigma$ we have $\alpha=\{\gamma \mid \gamma<\alpha\} \in \mathcal{L}$ and $\ell(\alpha)=X_{\alpha}$. 
(H2) If $\left(S_{i}\right)_{i \in I}$ is any family of elements of $\mathcal{L}$, then $\ell\left(\bigcup S_{i}\right)=\sum \ell\left(S_{i}\right)$ and $\ell\left(\bigcap S_{i}\right)=$ $\bigcap \ell\left(S_{i}\right)$. In particular, $\ell$ is a complete lattice homomorphism from $(\mathcal{L}, \cup, \cap)$ to the lattice $(\operatorname{Subobj}(X),+, \cap)$ of subobjects of $X$.

(H3) If $S, T \in \mathcal{L}$ are such that $S \subseteq T$, then the object $N=\ell(T) / \ell(S)$ is $\mathcal{S}$ filtered. More precisely, there is an $\mathcal{S}$-filtration $\left(N_{\beta} \mid \beta \leq \tau\right)$ and a bijection $b: T \backslash S \rightarrow \tau(=\{\beta \mid \beta<\tau\})$ such that $X_{\alpha+1} / X_{\alpha} \cong N_{b(\alpha)+1} / N_{b(\alpha)}$ for each $\alpha \in T \backslash S$.

(H4) For each $<\kappa$-generated subobject $Y \subseteq X$ there is $S \in \mathcal{L}$ of cardinality $<\kappa$ (so that $\ell(S)$ admits an $\mathcal{S}$-filtration of length $<\kappa$ by $(\mathrm{H} 3)$ ) such that $Y \subseteq$ $\ell(S) \subseteq X$.

Proof. The proof, which is rather technical, can be found in [67, Theorem 2.1]. While omitting the argument here, we shall at least indicate the construction of the class $\mathcal{L}$ and explain the main idea behind the proof in the simplest non-trivial case in Example 3.15. First we need to choose $\kappa$ given $\mathcal{G}$ and $\mathcal{S}$. The only condition for this is that $\mathcal{G}$ is locally $<\kappa$-presentable and each $Y \in \mathcal{S}$ is $<\kappa$-presented in the sense of $\left[2,25\right.$. Given the $\mathcal{S}$-filtration $\left(X_{\alpha} \mid \alpha \leq \sigma\right)$ of $X$, we can fix a family $\left(A_{\alpha}\right)_{\alpha<\sigma}$ of $<\kappa$-generated subobjects of $X$ (again in the sense of [25]) such that $X_{\alpha+1}=X_{\alpha}+A_{\alpha}$ for each $\alpha<\sigma$. Then we call a subset $S \subseteq \sigma$ closed if every $\alpha \in S$ satisfies

$$
X_{\alpha} \cap A_{\alpha} \subseteq \sum_{\substack{\gamma \in S, \gamma<\alpha}} A_{\gamma} .
$$

The set $\mathcal{L} \subseteq \mathcal{P}(\sigma)$ can be chosen as $\mathcal{L}=\{S \subseteq \sigma \mid S$ is closed $\}$ and the map $\ell$ assigns to $S \in \mathcal{L}$ the subobject $\sum_{\alpha \in S} A_{\alpha}$.

Example 3.15. Suppose that $R$ is a ring, $\mathcal{S}$ is a collection of finitely presented modules, and that $X \in \operatorname{Mod}-R$ has an $\mathcal{S}$-filtration of the form

$$
0=X_{0} \subseteq X_{1} \subseteq X_{2} \subseteq \cdots \subseteq X_{\omega} \subseteq X_{\omega+1}=X
$$

We will choose $\kappa=\aleph_{0}$ and fix a sequence of finitely generated submodules $\left(A_{\alpha}\right)_{\alpha \leq \omega}$, and $\mathcal{L}$ and $\ell$ as above. In order to prove (H4), suppose that $Y \subseteq X$ is finitely generated. If $Y \subseteq X_{\omega}$, then $Y \subseteq X_{n}$ for some $n<\omega$ and can put $S=\{0,1, \ldots, n-$ 1\}. If $Y \nsubseteq X_{\omega}$, we can without loss of generality assume that $A_{\omega} \subseteq Y$ since otherwise we could replace $Y$ by $Y+A_{\omega}$. Then we have a short exact sequence $0 \rightarrow X_{\omega} \cap Y \rightarrow Y \rightarrow X / X_{\omega} \rightarrow 0$ and so $X_{\omega} \cap Y$ is finitely generated by [70, Ch. 5, $\S 25]$. In particular there must exist $n_{0}<\omega$ such that $X_{\omega} \cap Y \subseteq X_{n_{0}}$ and the set $S=\left\{0,1, \ldots, n_{0}-1, \omega\right\}$ will do.

The significance of Proposition 3.14 is that, starting with one filtration of $X$, it allows us to construct many more filtrations of $X$. The crucial point is property (H4).

As a first application, we shall prove a theorem which provides us with a major source of examples of exact categories of Grothendieck type. 
Theorem 3.16. Let $\mathcal{G}$ be a Grothendieck category and $\mathcal{E} \subseteq \mathcal{G}$ be a deconstructible class which is closed under retracts. Then $\mathcal{E}$ together with the collection of all short exact sequences in $\mathcal{G}$ whose all terms belong to $\mathcal{E}$ (cf. Proposition 3.1) is an exact category of Grothendieck type.

Proof. (GT0) and (GT4) are clear from the definition, and (GT1) and (GT2) have been proved in [68, Lemma 1.11(i)]. The main difficulty remains in proving (GT3), the existence of a generator for $\mathcal{E}$.

Denote by $\mathcal{S}$ a set of objects of $\mathcal{E}$ such that $\mathcal{E}=$ Filt $\mathcal{S}$ and let $\kappa$ be a regular cardinal which is in accordance with Proposition 3.14. Let $\mathcal{E}_{\kappa}$ be the class of all objects in $\mathcal{G}$ which have an $\mathcal{S}$-filtration of length $<\kappa$. One observes that $\mathcal{E}_{\kappa}$ has only a set of representatives up to isomorphism, and we denote by $G$ the sum of all such representatives.

Clearly $G \in \mathcal{E}$ by Lemma 3.10 and we claim that $G$ is a generator for $\mathcal{E}$. We must prove that each $X \in \mathcal{E}$ admits a short exact sequence in $\mathcal{G}$ of the form

$$
0 \longrightarrow K \longrightarrow G^{(I)} \longrightarrow X \longrightarrow 0
$$

with $K \in \mathcal{E}$, and we shall do so by induction on the the least ordinal $\sigma=\sigma(X)$ such that $X$ has an $\mathcal{S}$-filtration of length $\sigma$ in $\mathcal{G}$. More precisely, given $X \in \mathcal{E}$ with an $\mathcal{S}$-filtration

$$
0=X_{0} \subseteq X_{1} \subseteq X_{2} \subseteq \cdots \subseteq X_{\alpha} \subseteq X_{\alpha+1} \subseteq \cdots \subseteq X_{\sigma}=X
$$

of length $\sigma$, we shall inductively construct in $\mathcal{G}$ a well-ordered direct system of short exact sequences

$$
\left(\varepsilon_{\alpha}: \quad 0 \longrightarrow K_{\alpha} \longrightarrow \coprod_{\beta<\alpha} G_{\beta} \stackrel{p_{\alpha}}{\longrightarrow} X_{\alpha} \longrightarrow 0\right)_{\alpha \leq \sigma}
$$

such that

(1) $K_{\alpha} \in \mathcal{E}$ for each $\alpha \leq \sigma$,

(2) $G_{\beta} \in \mathcal{E}_{\kappa}$ for each $\beta<\sigma$, and

(3) the vertical morphisms in the diagram

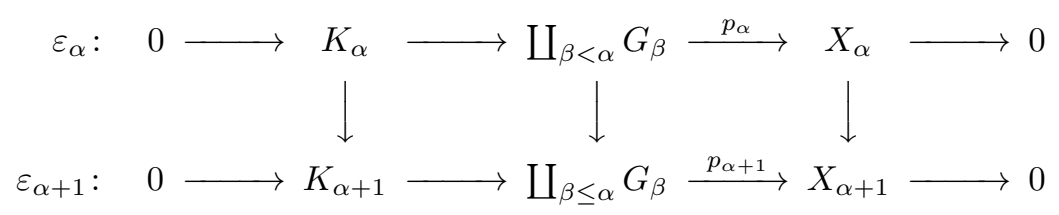

are monomorphisms for each $\alpha+1 \leq \sigma$. Moreover, the leftmost one has a cokernel in $\mathcal{E}$, the middle one is the canonical split monomorphism, and the rightmost one is the inclusion from the filtration of $X$. 
Before starting the construction, we shall fix a lattice map $\ell: \mathcal{L} \rightarrow \operatorname{Subobj}(X)$ as in Proposition 3.14. Now for $\sigma=0$ we start with the exact sequence of zeros and for the limit steps we just take the colimit sequences. Suppose that $\varepsilon_{\alpha}$ has been constructed and we are to construct $\varepsilon_{\alpha+1}$. By (H4) of Proposition 3.14, there is an element $S \in \mathcal{L} \subseteq \mathcal{P}(\sigma)$ such that $\alpha \in S$ and $|S|<\kappa$. Without loss of generality $S \subseteq(\alpha+1)$, since we can possibly replace $S$ by $S \cap(\alpha+1)$ thanks to (H1) and (H2). We shall put $G_{\alpha}=\ell(S)$ - this is up to isomorphism an element of $\mathcal{E}_{\kappa}$. Invoking (H1) and (H2) again, we have a bicartesian square of inclusions in $\mathcal{G}$ of the form

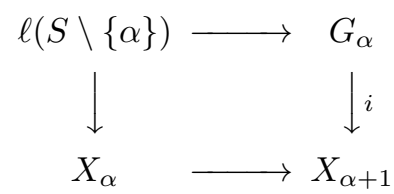

Let us define $K_{\alpha+1}$ by the pullback of $i: G_{\alpha} \rightarrow X_{\alpha+1}$ and the composition $t: \coprod_{\beta<\alpha} G_{\beta} \stackrel{p_{\alpha}}{\rightarrow} X_{\alpha} \stackrel{\subseteq}{\rightarrow} X_{\alpha+1}$ :

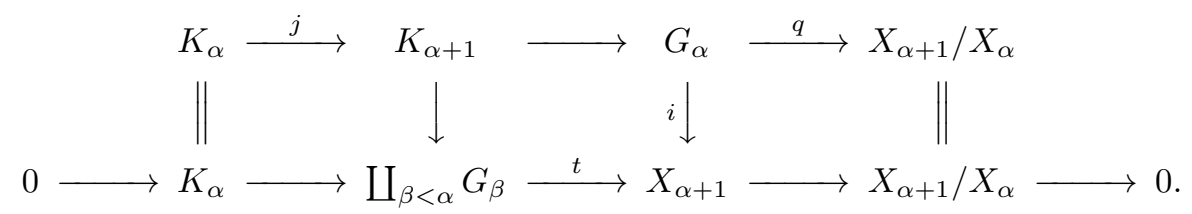

As $\operatorname{Im} t=X_{\alpha}$ and $\operatorname{Im} i \supseteq A_{\alpha}$ (using the notation from the proof of Proposition 3.14), we have $\operatorname{Im} t+\operatorname{Im} i=X_{\alpha+1}$ and we obtain a short exact sequence

$$
\varepsilon_{\alpha+1}: \quad 0 \longrightarrow K_{\alpha+1} \longrightarrow\left(\coprod_{\beta<\alpha} G_{\beta}\right) \oplus G_{\alpha} \stackrel{(t, i)}{\longrightarrow} X_{\alpha+1} \longrightarrow 0
$$

Further, $j: K_{\alpha} \rightarrow K_{\alpha+1}$ is clearly a monomorphism and it follows from the computations above that $q: G_{\alpha} \rightarrow X_{\alpha+1} / X_{\alpha}$ is an epimorphism. Now it only remains to prove that Coker $j \in \mathcal{E}$. Appealing to $(\dagger)$, one sees that $\operatorname{Ker} q \cong \ell(S \backslash\{\alpha\}) \in \mathcal{E}$, so that we have a short exact sequence

$$
0 \longrightarrow K_{\alpha} \stackrel{j}{\longrightarrow} K_{\alpha+1} \longrightarrow \ell(S \backslash\{\alpha\}) \longrightarrow 0,
$$

as required.

Example 3.17. Let $R$ be a ring, $\mathcal{G}=\operatorname{Mod}-R$ and $\mathcal{F}=$ Flat- $R$, the category of flat right $R$-modules. Then $\mathcal{F}$ satisfies the assumptions of Theorem 3.16 (see for instance [32, Lemma 3.2.7 and Theorem 4.1.1]) and so it is, with the exact structure induced from $\mathcal{G}$, an exact category of Grothendieck type. It came as a byproduct of Enoch's proof of the Flat Cover Conjecture [8] that $\mathcal{F}$ has enough injectives - these are usually called flat cotorsion modules.

We conclude the section with a few more properties of deconstructible classes, which among others show that the assumption on $\mathcal{E}$ being closed under retracts in Theorem 3.16 is not very restrictive. 
Lemma 3.18. 68, Lemma 1.11] Deconstructibility is transitive. Namely, let $\mathcal{E}$ be an exact category satisfying (Ef1) of Definition 3.4. If $\mathcal{F}$ is deconstructible in $\mathcal{E}$, then $\mathcal{F}$ also satisfies (Ef1) and, moreover, a class $\mathcal{F}^{\prime} \subseteq \mathcal{F}$ is deconstructible in $\mathcal{F}$ if and only if it is deconstructible in $\mathcal{E}$.

Proposition 3.19. 67, Proposition 2.9] Let $\mathcal{G}$ be a Grothendieck category, considered as an exact category with the abelian exact structure. Then:

(1) The closure of a deconstructible class of $\mathcal{G}$ under retracts is deconstructible.

(2) The intersection $\bigcap_{i \in I} \mathcal{F}_{i}$ of a collection of deconstructible classes $\left(\mathcal{F}_{i} \mid i \in I\right)$ of $\mathcal{G}$, indexed by a set $I$, is deconstructible.

\section{Weak factorization systems}

The coming section deals with a rather abstract category theory which will be of use later in the construction of cotorsion pairs and model structures. The highlight is a version of Quillen's small object argument, specialized to nice enough exact categories. We omit several technical steps in proofs and refer to the monographs [41, 38] and paper 65. Although most of our arguments are included in these references, the term "weak factorization system" itself is not. This term and some notation has been taken from 1 .

We start with an orthogonality relation on morphisms in an arbitrary category $\mathcal{C}$.

Definition 4.1. Given morphisms $f: A \rightarrow B$ and $g: X \rightarrow Y$ in $\mathcal{C}$, we write $f \square g$ if for any commutative square given by the solid arrows

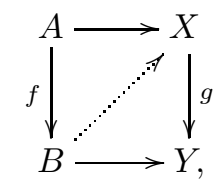

a morphism depicted by the diagonal dotted arrow exists such that both the triangles commute. We stress that we require only existence, not uniqueness of such a morphism. Note that if $\mathcal{C}$ is additive and we view $f$ and $g$ as complexes concentrated in two degrees, the orthogonality relation precisely says that every map from $f$ to $g$ is null-homotopic.

Given $f, g$ such that $f \square g$, we say that $f$ has the left lifting property for $g$ and $g$ has the right lifting property for $f$.

Now we can define the central concept of the section.

Definition 4.2. Let $\mathcal{C}$ be a category and $(\mathcal{L}, \mathcal{R})$ be a pair of classes of morphisms in $\mathcal{C}$. We say that $(\mathcal{L}, \mathcal{R})$ is a weak factorization system if 
(FS0) $\mathcal{L}$ and $\mathcal{R}$ are closed under retracts. That is, given any commutative diagram

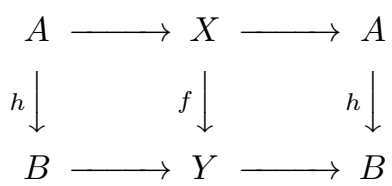

such that $f \in \mathcal{L}$ and the rows compose to the identity morphisms, then $h \in \mathcal{L}$ as well. We require the same for $\mathcal{R}$.

(FS1) $f \square g$ for all $f \in \mathcal{L}$ and $g \in \mathcal{R}$.

(FS2) For every morphism $h: X \rightarrow Y$ in $\mathcal{C}$, there is a factorization

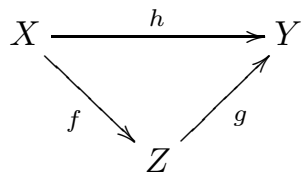

with $f \in \mathcal{L}$ and $g \in \mathcal{R}$.

We say that $(\mathcal{L}, \mathcal{R})$ is a functorial weak factorization system if the factorization in (FS2) can be chosen functorially in $h$.

Remark 4.3. Although the factorization as in (FS2), be it functorial or not, is typically non-unique in the cases we are concerned with, we can "compare" different factorizations using the lifting property. Namely, given $h=g f=\tilde{g} \tilde{f}$ such that $f, \tilde{f} \in \mathcal{L}$ and $g, \tilde{g} \in \mathcal{R},(\mathrm{FS} 1)$ in Definition 4.2 ensures that there is a morphism depicted by the dotted arrow making the diagram commutative:

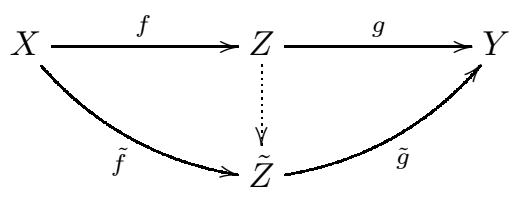

Example 4.4. A well-known example of a weak factorization system in an abelian category $\mathcal{A}$ is $(\mathcal{E}, \mathcal{M})$, where $\mathcal{E}$ is the class of all epimorphisms and $\mathcal{M}$ is the class of all monomorphisms. However, this example is rather misleading in our context. In Section [5], we will see weak factorization systems $(\mathcal{L}, \mathcal{R})$ where $\mathcal{L}$ is a class of monomorphisms and $\mathcal{R}$ is a class of epimorphisms.

Another fact is that $(\mathcal{E}, \mathcal{M})$ satisfies a stronger version of the lifting property from Definition 4.1 the diagonal morphism is unique for every square with $f \in \mathcal{E}$ and $g \in \mathcal{M}$, ensuring in view of Remark 4.3 that the factorizations as in (FS2) from Definition 4.2 are also unique. This is a strong property which our weak factorization systems typically do not enjoy.

A relatively easy observation concerning weak factorization systems is, that the two classes of morphisms determine each other. 
Lemma 4.5. Let $(\mathcal{L}, \mathcal{R})$ be a weak factorization system in a category $\mathcal{C}$. Then

$$
\mathcal{L}=\{f \mid f \square g \text { for all } g \in \mathcal{R}\} \quad \text { and } \quad \mathcal{R}=\{g \mid f \square g \text { for all } f \in \mathcal{L}\} .
$$

Proof. Clearly $\mathcal{L} \subseteq\{f \mid f \square g$ for all $g \in \mathcal{R}\}$ and we must prove the other inclusion. Take any $h: X \rightarrow Y$ such that $h \square g$ for all $g \in \mathcal{R}$ and consider a factorization

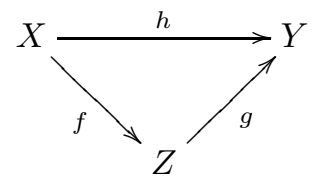

with $f \in \mathcal{L}$ and $g \in \mathcal{R}$. Since $h \square g$, the dotted arrow making the following diagram commutative exists

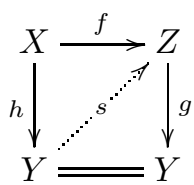

Placing the morphisms we have considered so far into the following diagram

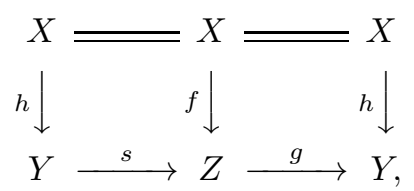

we observe that $g s=1_{Y}$. Thus, $h$ is a retract of $f$ and as such it must belong to $\mathcal{L}$.

The argument for $\mathcal{R}=\{g \mid f \square g$ for all $f \in \mathcal{L}\}$ is dual.

Now we can deduce closure properties of the left orthogonal of a class of morphisms with respect to $\square$. Note in particular that the left hand side class of any weak factorization system $(\mathcal{L}, \mathcal{R})$ has these properties.

Lemma 4.6. Let $\mathcal{R}$ be a class of morphisms in a category $\mathcal{C}$ and denote

$$
\mathcal{L}=\{f \mid f \square g \text { for each } g \in \mathcal{R}\}
$$

Then the following hold for $\mathcal{L}$ :

(1) $\mathcal{L}$ is closed under pushouts. That is, if we are given a diagram

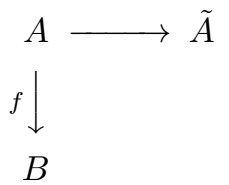


with $f \in \mathcal{L}$ and if the pushout

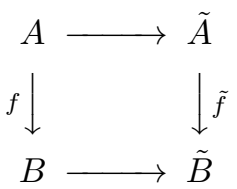

exists in $\mathcal{C}$, then also $\tilde{f} \in \mathcal{L}$.

(2) $\mathcal{L}$ is closed under transfinite compositions. That is, given a $\lambda$-sequence $\left(X_{\alpha}, f_{\alpha \beta}\right)_{\alpha<\beta<\lambda}$ with $f_{\alpha, \alpha+1} \in \mathcal{L}$ for every $\alpha+1<\lambda$, the composition $X_{0} \rightarrow \lim _{\alpha<\lambda} X_{\alpha}$, if it exists, belongs to $\mathcal{L}$, too.

Proof. This is an easy fact whose proof is left to the reader.

Inspired by the previous lemma, let us state another definition.

Definition 4.7. Given a set $\mathcal{I}$ of morphisms of a category $\mathcal{C}$, we define a relative $\mathcal{I}$-cell complex as a transfinite composition of pushouts of morphisms from $\mathcal{I}$. The class of all relative $\mathcal{I}$-cell complexes will be denoted by $\mathcal{I}$-cell.

We are now ready to state the highlight of the section-Quillen's small object argument. We state it first in a very general form taken from [65] and specialize it later. We will not give a full proof, but rather refer to the literature. A version of the argument can be found in 60 , while nice treatments with notation very close to ours can be found in 38,41 .

Theorem 4.8. Let $\mathcal{C}$ be a category and $\mathcal{M}$ be a class of morphisms such that

(1) Arbitrary pushouts of morphisms of $\mathcal{M}$ exist and belong again to $\mathcal{M}$.

(2) Arbitrary coproducts of morphisms of $\mathcal{M}$ exist and belong again to $\mathcal{M}$.

(3) Arbitrary transfinite compositions of maps of $\mathcal{M}$ exist and belong to $\mathcal{M}$.

Let $\mathcal{I} \subseteq \mathcal{M}$ be a set (not a proper class!) of morphisms and put

$$
\begin{aligned}
& \mathcal{R}=\{g \mid f \square g \text { for all } f \in \mathcal{I}\}, \\
& \mathcal{L}=\{f \mid f \square g \text { for all } g \in \mathcal{R}\} .
\end{aligned}
$$

If for every $i: A \rightarrow B$ in $\mathcal{I}$ the domain $A$ is small with respect to relative $\mathcal{I}$-cell complexes (Definition 3.3), then $(\mathcal{L}, \mathcal{R})$ is a functorial weak factorization system in $\mathcal{C}$ and $\mathcal{L}$ consists precisely of retracts of relative $\mathcal{I}$-cell complexes.

Proof. Clearly, conditions $(\mathrm{FS} 0)$ and $(\mathrm{FS} 1)$ of Definition 4.2 are satisfied for $(\mathcal{L}, \mathcal{R})$, so we only have to prove the functorial version of condition (FS2) and the structure of morphisms $\mathcal{L}$. Here we refer to [38, Proposition 10.5.16 and Corollary 10.5.22] or [41, Theorem 2.1.14 and Corollary 2.1.15]. Although the assumptions in 38, 41] differ slightly, identical proof works. 
Now we can specialize. First we state the correspondingly simplified version for Grothendieck categories.

Corollary 4.9. Let $\mathcal{G}$ be a Grothendieck category and $\mathcal{I}$ be a set of morphisms. If $(\mathcal{L}, \mathcal{R})$ is as in Theorem 4.8 , then $(\mathcal{L}, \mathcal{R})$ is a functorial weak factorization system in $\mathcal{G}$ and $\mathcal{L}$ consists precisely of retracts of relative $\mathcal{I}$-cell complexes.

Proof. Since $\mathcal{G}$ is cocomplete and has exact direct limits, hence also coproducts, by definition, we can take the class of all morphisms in $\mathcal{G}$ for $\mathcal{M}$. Regarding the smallness assumption on the domains of maps in $\mathcal{I}$, any object $A \in \mathcal{G}$ is small relative to the class all morphisms - see the proof of Proposition 3.13 .

Another corollary of Theorem 4.8 can be stated for efficient exact categories (Definition 3.4), or even slightly more generally without requiring the existence of a generator.

Corollary 4.10. Let $\mathcal{E}$ be an exact category such that

(1) Arbitrary transfinite compositions of inflations exist and are again inflations.

(2) Every object of $\mathcal{E}$ is small relative to the class of all inflations.

Let $\mathcal{I}$ be a set of inflations and consider $(\mathcal{L}, \mathcal{R})$ as in Theorem 4.8 . Then $(\mathcal{L}, \mathcal{R})$ is a functorial weak factorization system in $\mathcal{E}$ and $\mathcal{L}$ consists precisely of retracts of relative $\mathcal{I}$-cell complexes.

Proof. In this case, we can take for $\mathcal{M}$ the class of all inflations. Then $\mathcal{M}$ is closed under taking pushouts by the axioms of an exact category, and $\mathcal{M}$ is closed under coproducts by Lemma 3.5 .

\section{Complete cotorsion pairs}

In this section, we define complete cotorsion pairs and show how weak factorization systems are related to them. Along with this, we shall prove some properties of exact categories promised in Section 3 .

5.1. Definitions. Let us first briefly recall Yoneda's definition of Ext in exact categories, as our next discussion will be based on manipulation with these functors. We refer to [50, Chapter III and §XII.5] for a detailed account on the topic and further properties of Yoneda's Ext. Given an exact category $\mathcal{E}$, a sequence of morphisms

$$
\varepsilon: \quad 0 \longrightarrow Z_{n} \longrightarrow E_{n} \longrightarrow \cdots \longrightarrow E_{1} \longrightarrow Z_{0} \longrightarrow 0
$$

is called exact if it arises by splicing $n$ conflations in $\mathcal{E}$ of the form

$$
0 \longrightarrow Z_{i} \longrightarrow E_{i} \longrightarrow Z_{i-1} \longrightarrow 0 \text {. }
$$


Given further a pair of objects $X, Y \in \mathcal{C}$, let $E^{n}(X, Y)$ be the class of all exact sequences in $\mathcal{E}$ which are of the form

$$
\varepsilon: \quad 0 \longrightarrow Y \longrightarrow E_{n} \longrightarrow X \longrightarrow E_{1} \longrightarrow 0 .
$$

We define an equivalence relation $\sim$ on $E^{n}(X, Y)$ by relating $\varepsilon \sim \tilde{\varepsilon}$ whenever we have a commutative diagram of the form

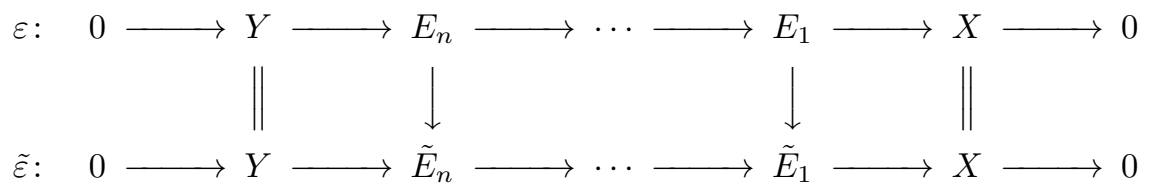

and taking the symmetric and transitive closure. The $n$-th Yoneda Ext of $X$ and $Y$ is defined as $\operatorname{Ext}_{\mathcal{E}}^{n}(X, Y)=E^{n}(X, Y) / \sim$. Although it is not a priori clear whether the Yoneda Ext is a set (and indeed in general it may be a proper class even for $n=1$, see [24, Exercise 1, p. 131]), we can nevertheless always give $\operatorname{Ext}_{\mathcal{E}}^{n}(X, Y) / \sim$ a structure of an abelian group using the so-called Baer sums and, whenever we are able to rule out the possible set theoretic pitfall,

$$
\operatorname{Ext}_{\mathcal{E}}^{n}(-,-): \mathcal{E}^{\mathrm{op}} \times \mathcal{E} \longrightarrow \mathrm{Ab}
$$

becomes an additive functor. Considering the case $n=1$, the zero element of $\operatorname{Ext}_{\mathcal{E}}^{1}(X, Y)$ is precisely the class of all split conflations.

Cotorsion pairs, originally defined in 64, are simply pairs of classes of objects mutually orthogonal with respect to $\operatorname{Ext}_{\mathcal{E}}^{1}(-,-)$.

Definition 5.1. Let $\mathcal{E}$ be an exact category. For a class $\mathcal{S}$ of objects of $\mathcal{E}$ we define

$$
\begin{aligned}
& \mathcal{S}^{\perp}=\left\{B \in \mathcal{E} \mid \operatorname{Ext}_{\mathcal{E}}^{1}(S, B)=0 \text { for all } S \in \mathcal{S}\right\} \\
& { }^{\perp} \mathcal{S}=\left\{A \in \mathcal{E} \mid \operatorname{Ext}_{\mathcal{E}}^{1}(A, S)=0 \text { for all } S \in \mathcal{S}\right\} .
\end{aligned}
$$

A pair $(\mathcal{A}, \mathcal{B})$ of full subcategories of $\mathcal{E}$ is called a cotorsion pair provided that

$$
\mathcal{A}={ }^{\perp} \mathcal{B} \quad \text { and } \quad \mathcal{A}^{\perp}=\mathcal{B} .
$$

A cotorsion pair $(\mathcal{A}, \mathcal{B})$ is said to be complete if every $X \in \mathcal{E}$ admits so-called approximation sequences; that is, conflations of the form

$$
0 \longrightarrow X \longrightarrow B_{X} \longrightarrow A_{X} \longrightarrow 0 \quad \text { and } \quad 0 \longrightarrow B^{X} \longrightarrow A^{X} \longrightarrow X \longrightarrow 0
$$

with $A_{X}, A^{X} \in \mathcal{A}$ and $B_{X}, B^{X} \in \mathcal{B}$.

The cotorsion pair is called functorially complete if, moreover, the approximation sequences can be chosen to be functorial in $X$.

Example 5.2. In every Grothendieck category $\mathcal{G}$ there is always a trivial complete cotorsion pair. Namely, denote by $\operatorname{Inj} \mathcal{G}$ the full subcategory of all injective objects. 
Then $(\mathcal{G}, \operatorname{Inj} \mathcal{G})$ is a complete cotorsion pair, where for given $X \in \mathcal{G}$ we have the following approximation sequences:

$$
0 \longrightarrow X \longrightarrow E X \longrightarrow E X / X \longrightarrow 0 \text { and } 0 \longrightarrow 0 \longrightarrow X \longrightarrow X \longrightarrow 0 \text {. }
$$

We denote by $X \rightarrow E X$ an injective envelope of $X$ (cf. Theorem 2.2). In fact, the trivial cotorsion pair is functorially complete, and as we shall see in Corollary [5.9. we have a similar result for exact categories of Grothendieck type.

5.2. Generators for the Ext functors. Next we shall develop a technical result necessary for the construction of complete cotorsion pairs using the small object argument from Section 4. A reader familiar with [42] will quickly notice the connection to the concept of a small cotorsion pair from [42, Definition 6.4]. As an easy consequence we will also show that given an efficient exact category (Definition 3.4), $\operatorname{Ext}_{\mathcal{E}}^{n}(X, Y)$ is a set for every choice of $X, Y \in \mathcal{E}$ and $n \geq 1$. Thus, no set-theoretic problems are to arise in our setting.

Proposition 5.3. Let $\mathcal{E}$ be an efficient exact category with a generator $G$, and let $S \in \mathcal{E}$ be an object. Then there exists a set $\mathcal{I}_{S}$ of inflations of $\mathcal{E}$ with the following properties:

(1) Each $f \in \mathcal{I}_{S}$ fits into a conflation

$$
0 \longrightarrow K \stackrel{f}{\longrightarrow} G^{(I)} \longrightarrow S \longrightarrow 0 .
$$

(2) If $h: X \rightarrow Y$ is an inflation in $\mathcal{E}$ with cokernel isomorphic to $S$, then $h$ is a pushout of some $f \in \mathcal{I}_{S}$. In other words, for each such h there exists $f \in \mathcal{I}_{S}$ so that we have a commutative diagram with conflations in rows:

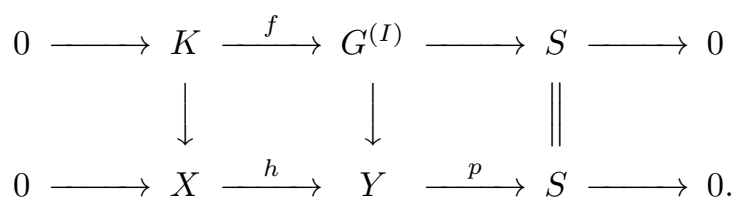

Proof. We shall give a direct construction of $\mathcal{I}_{S}$ and then prove that it has the properties we require. Consider a set $I \subseteq \mathcal{E}(G, S)$ and let

$$
p_{I}: G^{(I)} \longrightarrow S,
$$

be the canonical morphism - the one defined by $p_{I} \circ j_{i}=i$ for all $i \in I$, where $j_{i}: G \rightarrow G^{(I)}$ is the $i$-th coproduct injection. Let $\mathcal{D}_{S}$ be the collection of all $I \subseteq \mathcal{E}(G, S)$ such that $p_{I}$ is a deflation in $\mathcal{E}$. As $G$ is a generator, we have $\mathcal{E}(G, S) \in \mathcal{D}_{S}$ - for module and Grothendieck categories this is clear while for efficient exact categories this is shown in the proof of [65, Proposition 2.7].

We define $\mathcal{I}_{S}$ as the collection of the kernels $k_{I}$ of the morphisms $p_{I}$ for all $I \in \mathcal{D}_{S}$. That is, given $I \in \mathcal{D}_{S}$, we take a conflation

$$
0 \longrightarrow K_{I} \stackrel{k_{I}}{\longrightarrow} G^{(I)} \stackrel{p_{I}}{\longrightarrow} S \longrightarrow 0 .
$$


and include $k_{I}$ in $\mathcal{I}_{S}$.

As (1) is obviously satisfied for such an $\mathcal{I}_{S}$, we shall focus on (2). In fact here we give a proof only under the additional assumption that $\mathcal{E}$ is a Grothendieck category with the abelian exact structure. The fully general argument can be found in [65, Proposition 2.7] and it is similar, but more technical and not so enlightening.

Thus, consider a morphism $h: X \rightarrow Y$ which fits into a short exact sequence

$$
0 \longrightarrow X \stackrel{h}{\longrightarrow} Y \stackrel{p}{\longrightarrow} S \longrightarrow 0 .
$$

The object $G$ being a generator, there is an epimorphism $G^{(J)} \stackrel{g}{\rightarrow} Y$. Consider the composition $p g: G^{(J)} \rightarrow S$, which is necessarily an epimorphism too. Denoting by $\ell_{i}: G \rightarrow G^{(J)}$ the coproduct inclusions, we may get the same compositions $p g \ell_{i}: G \rightarrow S$ for distinct elements $i \in J$. We therefore define an equivalence relation on $J$ by putting

$$
i \sim i^{\prime} \text { for } i, i^{\prime} \in J \quad \text { if } \quad p g \ell_{i}=p g \ell_{i^{\prime}} .
$$

Let now $J^{\prime} \subseteq J$ be any set of representatives for the equivalence classes with respect to $\sim$, and define $g^{\prime}: G^{\left(J^{\prime}\right)} \rightarrow Y$ as the restriction of $g$ to $G^{\left(J^{\prime}\right)}$. We claim that the composition

$$
p g^{\prime}: G^{\left(J^{\prime}\right)} \longrightarrow S
$$

is still an epimorphism. Indeed, as the morphisms $p g \ell_{i}: G \rightarrow S$ for equivalent $i \in J$ contribute equally to the image of $p g$, we have

$$
\operatorname{Im} p g^{\prime}=\sum_{i \in J^{\prime}} \operatorname{Im} p g \ell_{i}=\sum_{i \in J} \operatorname{Im} p g \ell_{i}=\operatorname{Im} p g=S .
$$

This allows us to construct the diagram with exact rows as in (2), whose left hand side commutative square is necessarily bicartesian. We conclude the proof by noting that when identifying $J^{\prime}$ with a subset $I \subseteq \mathcal{E}(G, S)$ via the injective mapping $J^{\prime} \rightarrow \mathcal{E}(G, S)$ given by $i \mapsto p g^{\prime} \ell_{i}$, the kernel map $f$ of $p g^{\prime}$ belongs (up to isomorphism) to the set $\mathcal{I}_{S}$ constructed above.

It is not so difficult to extend the statement for $n$-fold extensions.

Corollary 5.4. Let $\mathcal{E}$ and $G$ be as in Proposition 5.3. Then any exact sequence

$$
0 \longrightarrow Y \longrightarrow E_{n} \longrightarrow \cdots \longrightarrow E_{1} \longrightarrow X \longrightarrow 0
$$

in $\mathcal{E}$ admits a commutative diagram with exact rows

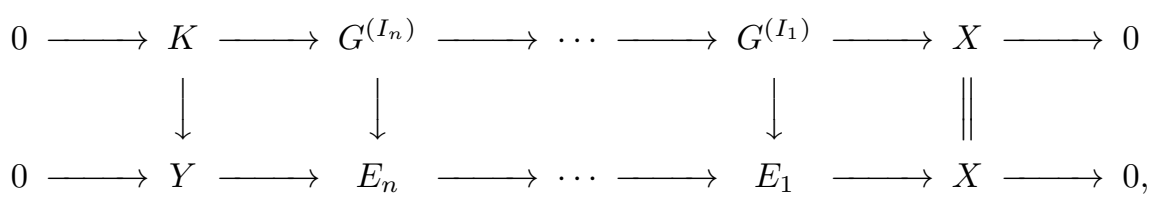

such that the cardinalities of $I_{1}, \ldots, I_{n}$ are bounded by a cardinal $\kappa=\kappa(X, G)$, which only depends on $X, G \in \mathcal{E}$. 
Proof. We prove the statement by induction in $n$. The case $n=1$ is clear from Proposition 5.3. If $n>1$, we use the inductive hypothesis to obtain a diagram

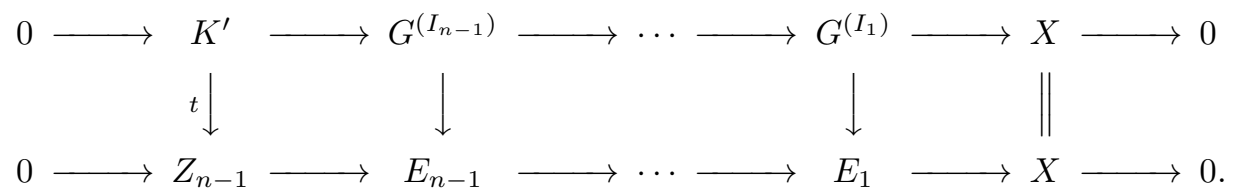

We also have the commutative diagram with conflations in rows

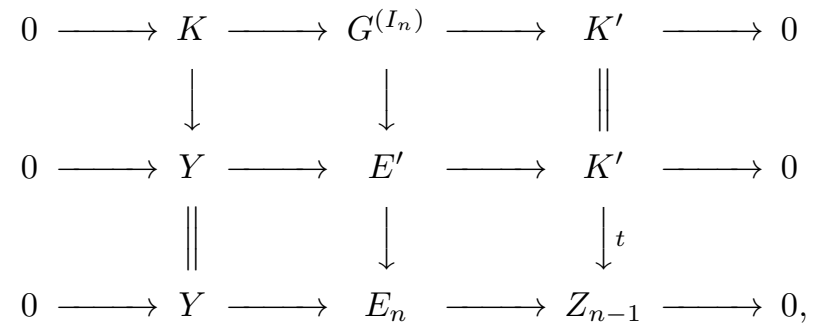

where the middle row is the pullback of the bottom row along $t$ and the upper row is obtained using Proposition 5.3 The diagram from the statement arises by ignoring the middle row in the last diagram and splicing it with the second last diagram.

As an easy corollary, we get the promised rectification of the potential settheoretic issue.

Corollary 5.5. Let $\mathcal{E}$ be an efficient exact category. Then $\operatorname{Ext}_{\mathcal{E}}^{n}(X, Y)$ is a set for each $X, Y \in \mathcal{E}$ and $n \geq 1$.

Proof. Let $\mathcal{E}$ and $G \in \mathcal{E}$ be as in Proposition 5.3 and fix $X \in \mathcal{E}$ and $n \geq 1$. Let $\kappa=\kappa(X, G)$ be a cardinal as in Corollary [5.5 and consider an exact sequence

$$
\varepsilon: \quad 0 \longrightarrow K_{\varepsilon} \longrightarrow G^{\left(I_{n}\right)} \longrightarrow \cdots \longrightarrow G^{\left(I_{1}\right)} \longrightarrow X \longrightarrow 0
$$

such that $\left|I_{1}\right|, \ldots,\left|I_{n}\right| \leq \kappa$. Given $Y \in \mathcal{E}$, there is an obvious map $\mathcal{E}\left(K_{\varepsilon}, Y\right) \rightarrow$ $\operatorname{Ext}_{\mathcal{E}}^{n}(X, Y)$ which acts by taking the pushouts of $\varepsilon$ along maps in $\mathcal{E}\left(K_{\varepsilon}, Y\right)$. The assignment is functorial in $Y$, so that we have a natural transformation $\mathcal{E}\left(K_{\varepsilon},-\right) \rightarrow$ $\operatorname{Ext}_{\mathcal{E}}^{n}(X,-)$. If we sum these transformations over all possible sequences $\varepsilon$, we get a transformation

$$
\coprod_{\varepsilon} \mathcal{E}\left(K_{\varepsilon},-\right) \longrightarrow \operatorname{Ext}_{\mathcal{E}}^{n}(X,-),
$$

which is surjective for every $Y \in \mathcal{E}$ by Corollary 5.4 .

5.3. From orthogonality on morphisms to vanishing of Ext. We start to work out the connection between complete cotorsion pairs and weak factorization systems and prove that exact categories of Grothendieck type have enough injectives. We shall start with two statements, which are taken from 65] in the generality in which we state them. 
Lemma 5.6. 65, 2.5] Let $\mathcal{E}$ be an efficient exact category, $G \in \mathcal{E}$ a generator and $S \in \mathcal{E}$ any fixed object. Consider the set $\mathcal{I}_{S}$ from Proposition 5.3. Then the following are equivalent for $Y \in \mathcal{E}$ :

(1) $\operatorname{Ext}_{\mathcal{E}}^{1}(S, Y)=0$.

(2) $f \square(Y \rightarrow 0)$ for every $f \in \mathcal{I}_{S}$ (cf. Definition 4.1).

Proof. (1) $\Longrightarrow(2)$ is easy and left to the reader. For $(2) \Longrightarrow(1)$, we will assume that $Y \rightarrow 0$ has the right lifting property for all $f \in \mathcal{I}_{S}$. We must prove that any fixed conflation

$$
\varepsilon: \quad 0 \longrightarrow Y \longrightarrow E \longrightarrow S \longrightarrow 0
$$

with $S \in \mathcal{S}$ splits. By the choice of $\mathcal{I}_{S}$, we know that we have the solid part of the commutative diagram

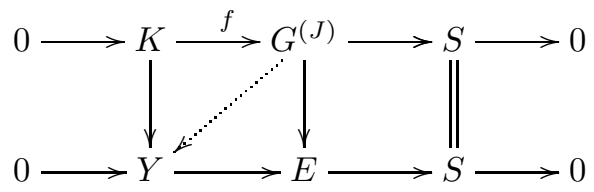

with conflations in rows and such that $f \in \mathcal{I}_{S}$. Since $f \square(Y \rightarrow 0)$, we can fill in the dotted arrow so that the upper triangle commutes. This precisely means that $\varepsilon$ splits.

The second result is, in a way, an analogue of Lemma 4.6(2) for cotorsion pairs. For modules, the corresponding result is called the Eklof lemma, see [32, Lemma 3.1.2]. We will present a rather different proof, however, which is taken from 65, Proposition 2.12].

Proposition 5.7. [65, 2.12] Let $\mathcal{E}$ be an exact category satisfying (Ef1) of Definition 3.4. Let $\mathcal{B}$ be a class of objects in $\mathcal{E}$ and denote $\mathcal{A}={ }^{\perp} \mathcal{B}$ (see Definition [5.1). Then any $\mathcal{A}$-filtered object belongs to $\mathcal{A}$.

Proof. Consider an $\mathcal{A}$-filtered object $X$ and $Y \in \mathcal{B}$. We must prove that any fixed extension

$$
\varepsilon: \quad 0 \longrightarrow Y \stackrel{j}{\longrightarrow} E \stackrel{p}{\longrightarrow} X \longrightarrow 0
$$

splits. Let us also fix an $\mathcal{A}$-filtration (see Definition 3.7)

$$
0=X_{0} \stackrel{f_{01}}{\longrightarrow} X_{1} \stackrel{f_{12}}{\longrightarrow} X_{2} \stackrel{f_{23}}{\longrightarrow} X_{3} \longrightarrow \cdots \longrightarrow X_{\omega} \stackrel{f_{\omega, \omega+1}}{\longrightarrow} X_{\omega+1} \longrightarrow \cdots
$$

for $X$ and let $\lambda$ be the ordinal by which this filtration is indexed. To facilitate the notation, we put $X_{\lambda}=X$.

Next we shall construct a $\lambda$-sequence of inflations

$$
Y=E_{0} \stackrel{j_{01}}{\longrightarrow} E_{1} \stackrel{j_{12}}{\longrightarrow} E_{2} \stackrel{j_{23}}{\longrightarrow} E_{3} \longrightarrow \cdots \longrightarrow E_{\omega} \stackrel{j_{\omega, \omega+1}}{\longrightarrow} E_{\omega+1} \longrightarrow \cdots
$$


whose transfinite composition is $j: Y \rightarrow E=E_{\lambda}$ and such that Coker $f_{\alpha, \alpha+1}=$ Coker $j_{\alpha, \alpha+1}$ for each $\alpha+1<\lambda$. If $\mathcal{E}$ is a Grothendieck category with the abelian exact structure, we just take the preimages $E_{\alpha}=p^{-1}\left(X_{\alpha}\right)$. In the general case, the construction is slightly more technical and we refer to [65, Lemma 2.10].

Finally we shall inductively construct a collection of morphisms $g_{\alpha}: E_{\alpha} \rightarrow Y$ such that $g_{0}=1_{Y}$ and for each $\alpha<\beta \leq \lambda$, the triangle

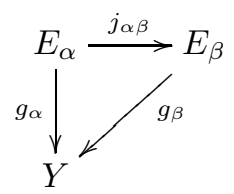

commutes. For $\alpha=0$ and $\beta=\lambda$, this precisely says that $\varepsilon$ splits since then $j_{0 \lambda}=j$.

Regarding the induction, we put $g_{0}=1_{Y}$ as required and at limit steps, we construct $g_{\alpha}$ as the colimit map of $\left(g_{\gamma}\right)_{\gamma<\alpha}$. For ordinal successors $\alpha+1 \leq \lambda$, suppose we have constructed $g_{\alpha}: E_{\alpha} \rightarrow Y$. We need to construct a factorization

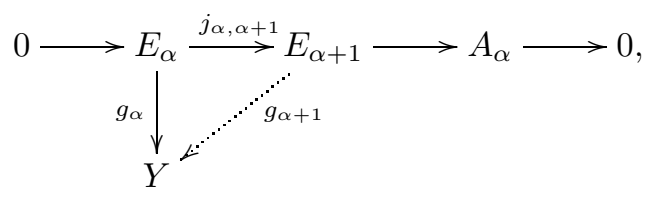

but this exists since $\operatorname{Ext}_{\mathcal{E}}^{1}\left(A_{\alpha}, Y\right)=0$.

Now we are in a position to give a criterion for the existence of one of the approximation sequences required for the completeness of a cotorsion pair in Definition 5.1

Proposition 5.8. [65, 2.13(4)] Let $\mathcal{E}$ be an efficient exact category, let $\mathcal{S}$ be a set (not a proper class!) of objects, and put $\mathcal{B}=\mathcal{S}^{\perp}$. Then there exist for every $X \in \mathcal{E}$ a short exact sequence

$$
0 \longrightarrow X \longrightarrow B_{X} \longrightarrow A_{X} \longrightarrow 0
$$

such that $B_{X} \in \mathcal{B}$ and $A_{X} \in$ Filt $\mathcal{S}\left(\subseteq{ }^{\perp} \mathcal{B}\right.$ by Proposition 5.7). The exact sequences can be chosen to be functorial in $X$.

Proof. Choose a generator $G$ for $\mathcal{E}$ and denote for each $S \in \mathcal{S}$ by $\mathcal{I}_{S}$ a set of inflations as in Proposition 5.3. Let $\mathcal{I}=\bigcup_{S \in \mathcal{S}} \mathcal{I}_{S}$ and put

$$
\mathcal{R}=\{g \mid f \square g \text { for all } f \in \mathcal{I}\} \quad \text { and } \quad \mathcal{L}=\{f \mid f \square g \text { for all } g \in \mathcal{R}\} .
$$

Then $(\mathcal{L}, \mathcal{R})$ is a functorial weak factorization system by Corollary 4.10, If $X \in \mathcal{E}$ is an arbitrary object, we have a functorial factorization

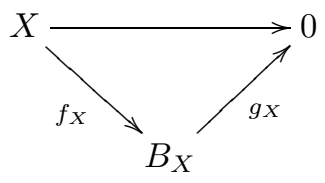


with $f_{X} \in \mathcal{I}$-cell (see Definition 4.7) and $g_{X} \in \mathcal{R}$. Now $B_{X} \in \mathcal{B}$ by Lemma 5.6 and $f_{X}$ is an inflation with $\mathcal{S}$-filtered cokernel by (Ef1) of Definition 3.4 Hence we have functorial short exact sequences $0 \longrightarrow X \stackrel{f_{X}}{\longrightarrow} B_{X} \longrightarrow A_{X} \longrightarrow 0$ as required.

Now we can prove another aforementioned result - the existence of enough injectives for exact categories of Grothendieck type. Recall that $I \in \mathcal{E}$ is called injective if $\operatorname{Ext}_{\mathcal{E}}^{1}(-, I) \equiv 0$. Denote the class of all injective objects by $\operatorname{Inj} \mathcal{E}$.

Corollary 5.9. Let $\mathcal{E}$ be an exact category of Grothendieck type (Definition 3.11). Then $(\mathcal{E}, \operatorname{Inj} \mathcal{E})$ is a functorially complete cotorsion pair.

Proof. Fix a set $\mathcal{S} \subseteq \mathcal{E}$ such that $\mathcal{E}=$ Filt $\mathcal{S}$-we can do that thanks to (GT4) of Definition 3.11. Then $\mathcal{S}^{\perp}=\operatorname{Inj} \mathcal{E}$ by Proposition [5.7 and, by Proposition [5.8, there are exact sequences

$$
0 \longrightarrow X \longrightarrow E(X) \longrightarrow \mho(X) \longrightarrow 0
$$

functorial in $X$ such that $E(X) \in \operatorname{Inj} \mathcal{E}$. The approximation sequences of the other type (Definition [5.1) are trivial, see Example 5.2.

Remark 5.10. We cannot really speak of an injective cogenerator in general since exact categories of Grothendieck type may not have products. For instance, $\mathcal{E}=$ Flat- $R$ from Example 3.17 has products if and only if $R$ is left coherent by [12, Theorem 2.1]. This deficiency, however, does not pose any problems here.

5.4. Weak factorization systems versus cotorsion pairs. Now we are going to relate cotorsion pairs to weak factorization systems compatible with the exact structure. As a consequence, we prove a vast generalization of the criterion for completeness of cotorsion pairs from [15]. The ideas are mostly taken from [42, $\S \S 4-$ $5]$ and 62. A similar presentation has been later given, specifically for categories of complexes of modules, in [21, Chapter 6].

Let us make precise in which way we want our weak factorization system $(\mathcal{L}, \mathcal{R})$ to be compatible with the exact structure. Note first that $f \in \mathcal{L}$ is an inflation, then $0 \rightarrow$ Coker $f$ belongs to $\mathcal{L}$ by Lemma 4.6(1). Dually, if $f \in \mathcal{R}$ is a deflation, then $\operatorname{Ker} f \rightarrow 0$ is in $\mathcal{R}$. Using the terminology in accordance with 31 we define:

Definition 5.11. Let $\mathcal{E}$ be an arbitrary exact category and $(\mathcal{L}, \mathcal{R})$ be a weak factorization system. We call $(\mathcal{L}, \mathcal{R})$ an exact weak factorization system if the following two conditions are satisfied for a morphism $f$ in $\mathcal{E}$ :

(FS3) $f \in \mathcal{L}$ if and only if $f$ is an inflation and $0 \rightarrow$ Coker $f$ belongs to $\mathcal{L}$.

(FS4) $f \in \mathcal{R}$ if and only if $f$ is an deflation and $\operatorname{Ker} f \rightarrow 0$ belongs to $\mathcal{R}$.

Exact functorial weak factorization systems are defined analogously.

Thus, an exact weak factorization system is determined by a pair of classes of objects - the cokernels of morphisms in $\mathcal{L}$ and the kernels of morphisms in $\mathcal{R}$. Before stating the main result of the section, we shall introduce notations for this correspondence. 
Notation 5.12. Given a class $\mathcal{L}$ of inflations, we put Coker $\mathcal{L}=\{A \mid A \cong$ Coker $f$ for some $f \in \mathcal{L}\}$. Dually for a class $\mathcal{R}$ of deflations, Ker $\mathcal{R}=\{B \mid B \cong$ Ker $f$ for some $f \in \mathcal{R}\}$.

Conversely, given classes of objects $\mathcal{A}, \mathcal{B} \subseteq \mathcal{E}$, we denote by $\operatorname{Infl} \mathcal{A}$ the class of all inflations with cokernel in $\mathcal{A}$, and by Defl $\mathcal{B}$ the class of all deflations with kernel in $\mathcal{B}$.

Theorem 5.13. Let $\mathcal{E}$ be an exact category. Then

$$
(\mathcal{L}, \mathcal{R}) \longmapsto(\text { Coker } \mathcal{L}, \operatorname{Ker} \mathcal{R}) \quad \text { and } \quad(\mathcal{A}, \mathcal{B}) \mapsto(\operatorname{Infl} \mathcal{A}, \text { Defl } \mathcal{B})
$$

define mutually inverse bijective mappings between exact weak factorization systems $(\mathcal{L}, \mathcal{R})$ and complete cotorsion pairs $(\mathcal{A}, \mathcal{B})$. The bijections restrict to mutually inverse mappings between exact functorial weak factorization systems $(\mathcal{L}, \mathcal{R})$ and functorially complete cotorsion pairs $(\mathcal{A}, \mathcal{B})$.

Before proving the theorem, we shall establish two auxiliary lemmas, which should be rather self-explanatory.

Lemma 5.14. Let $\mathcal{E}$ be an exact category, $f$ be an inflation and $g$ be a deflation in $\mathcal{E}$. If $\operatorname{Ext}_{\mathcal{E}}^{1}(\operatorname{Coker} f, \operatorname{Ker} g)=0$, then $f \square g$.

Proof. Suppose that we have a commutative square formed by the solid arrows

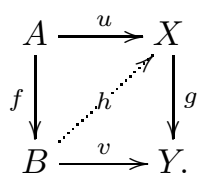

We must prove the existence of the dotted morphism $h: B \rightarrow X$. If we denote $C=\operatorname{Coker} f$ and $K=\operatorname{Ker} g$, we obtain the following commutative diagram of abelian groups with exact rows:

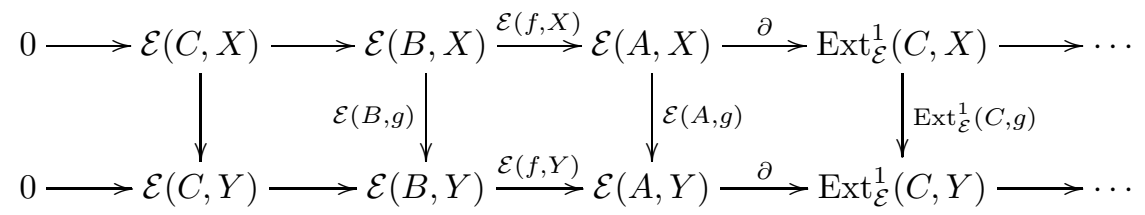

If we view the diagram as a map of complexes, the mapping cone

$$
\cdots \rightarrow \mathcal{E}(B, X) \oplus \mathcal{E}(C, Y) \rightarrow \mathcal{E}(A, X) \oplus \mathcal{E}(B, Y) \stackrel{\partial^{\prime}}{\rightarrow} \operatorname{Ext}_{\mathcal{E}}^{1}(C, X) \oplus \mathcal{E}(A, Y) \rightarrow \cdots
$$

must be exact as well by [50, Proposition II.4.3]. Given the construction of the cone, we only need to prove that $\partial^{\prime}(-u, v)=0$. This amounts to showing that

$$
-\partial(-u)=0 \quad \text { and } \quad \mathcal{E}(f, Y)(v)+\mathcal{E}(A, g)(-u)=0 .
$$


The second equality holds since $g u=v f$, while for the first one we use the equalities

$$
\operatorname{Ext}_{\mathcal{E}}^{1}(C, g)(\partial(-u))=\partial(\mathcal{E}(A, g)(-u))=\partial(\mathcal{E}(f, Y)(-v))=0
$$

and the fact that $\operatorname{Ext}_{\mathcal{E}}^{1}(C, g)$ is a monomorphism because of

$$
0=\operatorname{Ext}_{\mathcal{E}}^{1}(C, K) \longrightarrow \operatorname{Ext}_{\mathcal{E}}^{1}(C, X) \stackrel{\operatorname{Ext}_{\mathcal{E}}^{1}(C, g)}{\longrightarrow} \operatorname{Ext}_{\mathcal{E}}^{1}(C, Y)
$$

Lemma 5.15. Let $\mathcal{E}$ be an exact category. If $\mathcal{A} \subseteq \mathcal{E}$ is a class of objects and $g$ is a deflation such that $f \square g$ for each inflation $f$ with Coker $f \in \mathcal{A}$, then $\operatorname{Ker} g \in \mathcal{A}^{\perp}$. Dually if $\mathcal{B} \subseteq \mathcal{E}$ and $f$ is an inflation such that $f \square g$ for each deflation $g$ with Ker $g \in \mathcal{B}$, then Coker $f \in{ }^{\perp} \mathcal{B}$.

Proof. Suppose that $g: X \rightarrow Y$ is a deflation such that $f \square g$ for each inflation $f$ with Coker $f \in \mathcal{A}$. Denote $K=\operatorname{Ker} g$ and consider a conflation

$$
\varepsilon: \quad 0 \longrightarrow K \stackrel{i}{\longrightarrow} E \longrightarrow A \longrightarrow 0
$$

with $A \in \mathcal{A}$. Then in particular $i \square g$ and by the dual of Lemma 4.6(1) we also have $i \square(K \rightarrow 0)$. Applying the latter fact to the commutative square

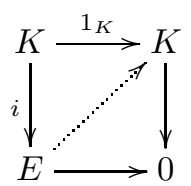

we see that $\varepsilon$ splits. Since $\varepsilon$ was chosen arbitrarily, we have $K \in \mathcal{A}^{\perp}$. The other part of the lemma is dual.

Now we can finish the proof of the theorem.

Proof of Theorem 5.13. Suppose first that $(\mathcal{L}, \mathcal{R})$ is an exact weak factorization system and put $\mathcal{A}=\operatorname{Coker} \mathcal{L}$ and $\mathcal{B}=\operatorname{Ker} \mathcal{R}$. Both $\mathcal{A}$ and $\mathcal{B}$ are closed under retracts since so are $\mathcal{L}$ and $\mathcal{R}$, and $\operatorname{Ext}_{\mathcal{E}}^{1}(A, B)=0$ for each $A \in \mathcal{A}$ and $B \in \mathcal{B}$ by Lemma 5.15. In order to prove the existence of approximation sequences from Definition 5.1 consider $X \in \mathcal{E}$ and the following two factorizations
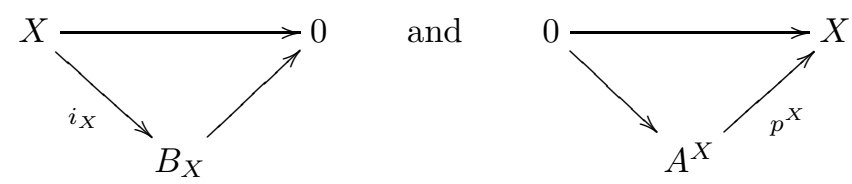

with respect to $(\mathcal{L}, \mathcal{R})$. It follows directly from Definition 5.11 that there are conflations

$$
0 \longrightarrow X \stackrel{i_{X}}{\longrightarrow} B_{X} \longrightarrow A_{X} \longrightarrow 0 \quad \text { and } \quad 0 \longrightarrow B^{X} \longrightarrow A^{X} \stackrel{p^{X}}{\longrightarrow} X \longrightarrow 0
$$


with $A_{X}, A^{X} \in \mathcal{A}$ and $B_{X}, B^{X} \in \mathcal{B}$. Finally, if $X \in{ }^{\perp} \mathcal{B}$ then the second approximation sequence splits and so $X \in \mathcal{A}$. Similarly $\mathcal{B}=\mathcal{A}^{\perp}$.

Let conversely $(\mathcal{A}, \mathcal{B})$ be a complete cotorsion pair in $\mathcal{E}$, and put $\mathcal{L}=\operatorname{Infl} \mathcal{A}$ and $\mathcal{R}=\operatorname{Defl} \mathcal{B}$. Then $\mathcal{L}$ and $\mathcal{R}$ are closed under retracts since so are $\mathcal{A}$ and $\mathcal{B}$. If $f \in \mathcal{L}$ and $g \in \mathcal{R}$, then $f \square g$ by Lemma 5.14.

Next we shall prove that every morphism $h: X \rightarrow Y$ factorizes as $h=g f$ with $f \in \mathcal{L}$ and $g \in \mathcal{R}$. Suppose first that $h$ is an inflation and consider the following pullback diagram with an approximation sequence for $C=\operatorname{Coker} f$ in the rightmost column.

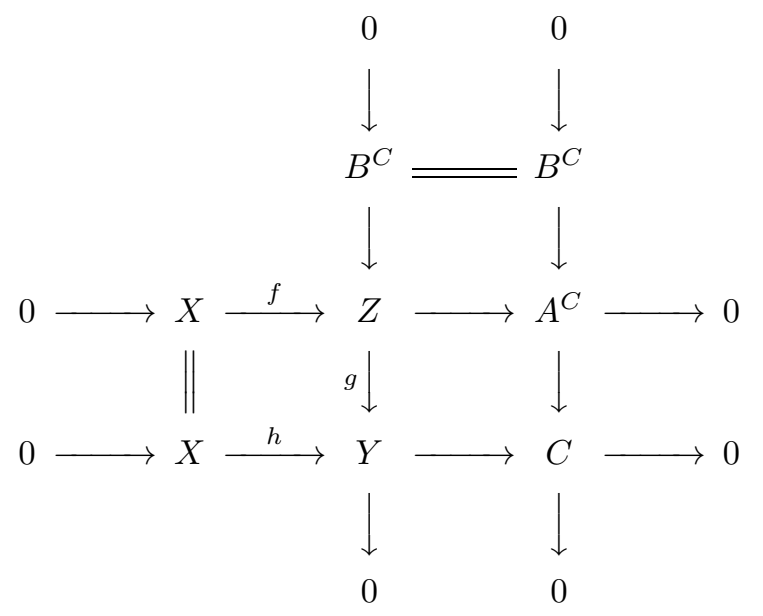

The corresponding factorization of $h$ then appears in the leftmost square. A dual argument applies if $h$ is a deflation. If $h$ is an arbitrary morphism, we can functorially factorize it as

$$
X \stackrel{\text { inc }}{\longrightarrow} X \oplus Y \stackrel{\left(h, 1_{Y}\right)}{\longrightarrow} Y .
$$

As $X \rightarrow X \oplus Y$ is a split inflation, we can factor it as inc $=g_{1} f_{1}$ with $f_{1} \in \mathcal{L}$ and $g_{1} \in \mathcal{R}$. Now the composition $\left(h, 1_{Y}\right) \circ g_{1}$ is a deflation, so that we can factor it as $g_{2} f_{2}$ with $f_{2} \in \mathcal{L}$ and $g_{2} \in \mathcal{R}$ :

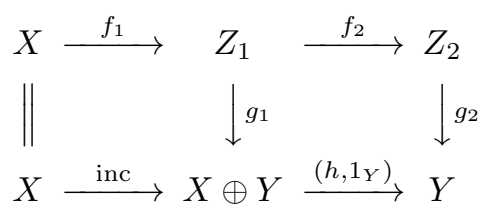

It follows that $h=g_{2}\left(f_{2} f_{1}\right)$ and that $f_{2} f_{1} \in \mathcal{L}=\operatorname{Infl} \mathcal{A}$ by [10, Lemma 3.5] since $\mathcal{A}$ is closed under extensions.

Conditions (FS3) and (FS4) from Definition $[5.11$ are clearly satisfied by the very definition of $(\mathcal{L}, \mathcal{R})$. Thus, $(\mathcal{L}, \mathcal{R})$ is an exact weak factorization system.

Finally, the passage from factorization of morphisms to approximation sequences and back is clearly functorial. This proves the last claim.

As a consequence, we can improve Proposition 5.8 and recover an existence result for complete cotorsion pairs from [65, §2], which vastly generalizes the main 
result of [15. The argument itself can be traced back to [42, Theorem 6.5] and 62. To underline the significance of this type of result, we note that [15] was one of the starting points of the monograph 32, which discusses various results and techniques to study infinitely generated modules. Taking into account our Section 2 , many of the techniques carry over to the categories of quasi-coherent sheaves directly.

Theorem 5.16. Let $\mathcal{E}$ be an efficient category and $\mathcal{S}$ be a set (not a proper class!) of objects such that Filt $\mathcal{S}$ contains a generator for $\mathcal{E}$. Put

$$
\mathcal{B}=\mathcal{S}^{\perp} \quad \text { and } \quad \mathcal{A}={ }^{\perp} \mathcal{B} .
$$

Then $(\mathcal{A}, \mathcal{B})$ is a functorially complete cotorsion pair in $\mathcal{E}$ (Definition 5.1) and $\mathcal{A}$ consists precisely of retracts of $\mathcal{S}$-filtered objects.

Proof. As in the proof of Proposition 5.8, we choose a generator $G$ for $\mathcal{E}$ and denote for each $S \in \mathcal{S}$ by $\mathcal{I}_{S}$ a set of inflations given by Proposition 5.3 . We further put $\mathcal{I}=\bigcup_{S \in \mathcal{S}} \mathcal{I}_{S}$ and

$$
\mathcal{R}=\{g \mid f \square g \text { for all } f \in \mathcal{I}\} \quad \text { and } \quad \mathcal{L}=\{f \mid f \square g \text { for all } g \in \mathcal{R}\},
$$

obtaining a functorial weak factorization system $(\mathcal{L}, \mathcal{R})$.

Denote by $\mathcal{F}$ the closure of Filt $\mathcal{S}$ (see Definition 3.7) under retracts. Thanks to the construction of $\mathcal{I}$, a morphisms $f^{\prime}$ is an inflation with a cokernel from $\mathcal{S}$ if and only if it is a pushout of some $f \in \mathcal{I}$. It follows from the description of $\mathcal{L}$ in Corollary 4.10 that $\mathcal{L}=\operatorname{Infl} \mathcal{F}$.

We claim that $\mathcal{R}$ consists of deflations with kernels in $\mathcal{B}$. Let $g: X \rightarrow Y$ be in $\mathcal{R}$ and consider a deflation $p: F \rightarrow Y$ with $F \in \mathcal{F}$ - such a $p$ must exist since $\mathcal{F}$ contains a generator. Then the dotted arrow in the square

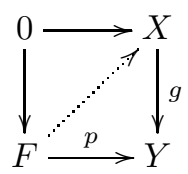

can be filled in in such a way that both triangles commute. Since $\mathcal{E}$ is weakly idempotent complete, $g$ is a deflation by Lemma 3.6(2). Then Ker $g \in \mathcal{A}^{\perp}=\mathcal{B}$ by Lemmas 5.15 and 4.6(1), finishing the proof of the claim.

In fact, we even have $\mathcal{R}=\operatorname{Defl} \mathcal{B}$ by Lemma 5.14. Hence $(\mathcal{F}, \mathcal{B})$ is a functorially complete cotorsion pair by Theorem 5.13 and necessarily $\mathcal{F}=\mathcal{A}$, which concludes the proof.

As a consequence we obtain a convenient criterion for recognizing the left hand side class of a functorially complete cotorsion pair.

Corollary 5.17. Let $\mathcal{E}$ be an efficient exact category and $\mathcal{F} \subseteq \mathcal{C}$ be a class of objects which is deconstructible, closed under retracts and contains a generator. Then $\left(\mathcal{F}, \mathcal{F}^{\perp}\right)$ is a functorially complete cotorsion pair in $\mathcal{E}$. 
Proof. If $\mathcal{S}$ is a set of objects such that $\mathcal{F}=$ Filt $\mathcal{S}$, then $\mathcal{F}={ }^{\perp}\left(\mathcal{S}^{\perp}\right)$ by Theorem 5.16

A partial converse holds for Grothendieck categories, or more generally for exact categories of Grothendieck type which arise as in Theorem 3.16.

Corollary 5.18. Let $\mathcal{E}$ be a deconstructible subcategory closed under retracts in a Grothendieck category, with the induced exact structure (hence $\mathcal{E}$ is of Grothendieck type). If $(\mathcal{A}, \mathcal{B})$ is a (functorially) complete cotorsion pair such that $\mathcal{B}=\mathcal{S}^{\perp}$ for a set $\mathcal{S}$, then $\mathcal{A}$ is deconstructible, closed under retracts and contains a generator.

Proof. The only problem consists in proving that $\mathcal{A}$ is deconstructible, as we know that $\mathcal{A}$ is the collection of all retracts of objects in Filt $\mathcal{S}$. But this follows from Proposition 3.19(1) and Lemma 3.18. For the sake of completeness, we note that the proof of Proposition 3.19 in 67] uses the Hill Lemma (Proposition 3.14).

\section{Exact and hereditary model categories}

In this section we shall give an account on Quillen model categories. The motivation for model categories is the following situation. We start with a category $\mathcal{C}$ and a class of morphisms $\mathrm{W}$, and we would like to understand the category $\mathcal{C}\left[\mathrm{W}^{-1}\right]$ where the morphisms in $\mathrm{W}$ are made artificially invertible. It is not difficult to construct such $\mathcal{C}\left[\mathrm{W}^{-1}\right]$ formally (see [26]), up to a set-theoretic difficulty. Namely, the collections of morphisms $\mathcal{C}\left[\mathrm{W}^{-1}\right](X, Y)$ may be proper classes rather than sets - see [1] for an example of this pathology. If $(\mathcal{C}, W)$ admits some extra structure making it a model category, the latter difficulty disappears, but there are more advantages as we shall see.

After recalling the classical properties of model categories, we aim at showing how model categories can be obtained using the tools from previous sections. The idea here is due to Hovey [42].

6.1. The homotopy category of a model category. First, we shall briefly recall the general theory, as at this point this can be done rather quickly and efficiently. Standard sources for more information about the topic are [38, 41].

Definition 6.1. Let $\mathcal{C}$ be a category. A model structure on $\mathcal{C}$ is a triple (Cof, W, Fib) of classes of morphisms, called cofibrations, weak equivalences and fibrations, respectively, such that

(MS1) W is closed under retracts and satisfies the 2-out-of-3 property for composition. That is, if $f, g$ is a pair of composable morphisms in $\mathcal{C}$ and two of $f, g, g f$ are in $\mathrm{W}$, so is the third.

(MS2) (Cof, $\mathrm{W} \cap \mathrm{Fib}$ ) and (Cof $\cap \mathrm{W}$, Fib) are weak factorization systems in $\mathcal{C}$.

Morphisms in Cof $\cap \mathrm{W}$ are called trivial cofibrations and morphisms in $\mathrm{W} \cap \mathrm{Fib}$ are trivial fibrations. 
A model category is a category with a model structure such that $\mathcal{C}$ has an initial object $\emptyset$, a terminal object $*$, all pushouts of trivial cofibrations along trivial cofibrations exists, and dually all pullbacks of trivial fibrations along trivial fibrations exist.

Remark 6.2. The definition of a model structure on $\mathcal{C}$, although seemingly different, is equivalent to [41, Definition 1.1.3] up to one detail. We have dropped the functoriality of the weak factorization systems from (MS2). This subtle change is inessential for the theory discussed here and we have mostly done the change to be able to use Proposition [7.7. On the other hand, almost all weak factorization systems available in practice seem to be generated by a set of morphisms from the left hand side as in Theorem 4.8 (such model structures are usually called cofibrantly generated, [38, 11.1.2]), so they functorial.

Our definition of a model category differs slightly from [38, Definition 7.1.3] and [41, Definition 1.1.4] in that we do not require existence of all limits and colimits in $\mathcal{C}$, but only $\emptyset$, $*$, and certain pushouts and pullbacks. The reason for this digression is that exact categories of Grothendieck type are often not complete and cocomplete (see Example [3.17), but our assumptions are enough for the fundamental Theorem 6.7 below. Moreover, if $\mathcal{C}$ is an exact category and the two weak factorization systems in (MS2) are exact, then the existence of the pushouts and pullbacks which we need is implicit in the definition of an exact category, so that we even need no additional assumptions on (co)completeness of $\mathcal{C}$ !

If we have a model category, the existence of the initial and terminal object allows us to define cofibrant and fibrant objects of $\mathcal{C}$, as well as cofibrant and fibrant "approximations" of any object.

Definition 6.3. An object $B \in \mathcal{C}$ is called cofibrant (trivially cofibrant) if $\emptyset \rightarrow B$ is a cofibration (trivial cofibration, resp.) Dually $X \in \mathcal{C}$ is fibrant (trivially fibrant) if $X \rightarrow *$ is a fibration (trivial fibration, resp.)

If $X \in \mathcal{C}$ is arbitrary and $\emptyset \rightarrow C X \rightarrow X$ is a factorization of $\emptyset \rightarrow X$ with respect to (Cof, $\mathrm{W} \cap \mathrm{Fib}$ ), then $C X$ is called a cofibrant replacement of $X$. Dually, the object $F X$ in a factorization $X \rightarrow F X \rightarrow *$ with respect to (Cof $\cap$ W, Fib) is called a fibrant replacement of $X$.

It is further useful to notice that a model structure is overdetermined in that two of Cof, W, Fib determine the third. Before discussing the homotopy category of a model category, we need to briefly recall the homotopy relations.

Definition 6.4. [41, 1.2.4] Let $\mathcal{C}$ be a model category and $f, g: B \rightarrow X$ be two morphisms in $\mathcal{C}$.

(1) A cylinder object for $B$ is a factorization of the codiagonal map $\nabla: B \amalg B \rightarrow B$ into a cofibration $\left(i_{0}, i_{1}\right)$ followed by a weak equivalence $s$ :

$$
B \amalg B \stackrel{\left(i_{0}, i_{1}\right)}{\longrightarrow} B^{\prime} \stackrel{s}{\longrightarrow} B .
$$


(2) A path object for $X$ is dually a factorization of the diagonal map $\Delta: X \rightarrow$ $X \times X$ into a weak equivalence followed by a fibration:

$$
X \stackrel{r}{\longrightarrow} X^{\prime} \stackrel{\left(\begin{array}{l}
p_{0} \\
p_{1}
\end{array}\right)}{\longrightarrow} X \times X .
$$

(3) A left homotopy from $f$ to $g$ is a map $H: B^{\prime} \rightarrow X$ for some cylinder object $B^{\prime}$ for $B$ such that such that $H i_{0}=f$ and $H i_{1}=g$. We say that $f$ and $g$ are left homotopic, written $f \sim_{\ell} g$, if there is a left homotopy from $f$ to $g$.

(4) A right homotopy from $f$ to $g$ is a map $K: B \rightarrow X^{\prime}$ for some path object $X^{\prime}$ for $X$ such that such that $p_{0} K=f$ and $p_{1} K=g$. We say that $f$ and $g$ are right homotopic, $f \sim_{r} g$, if there is a right homotopy from $f$ to $g$.

(5) Morphisms $f$ and $g$ are said to be homotopic, written $f \sim g$, if they are both left and right homotopic.

Let us summarize the relevant properties of the homotopy relations.

Proposition 6.5. Let $\mathcal{C}$ be a model category and suppose that $A, B \in \mathcal{C}$ are cofibrant and $X, Y \in \mathcal{C}$ are fibrant. Then the following hold:

(1) Morphisms $f, g: B \rightarrow X$ are left homotopic if and only if they are right homotopic if and only if they are homotopic.

(2) The homotopy is an equivalence relation on $\mathcal{C}(B, X)$.

(3) If $h: A \rightarrow B$ is a morphism and $f \sim g$, then $f h \sim g h$.

(4) If $h^{\prime}: X \rightarrow Y$ is a morphism and $f \sim g$, then $h^{\prime} f \sim h^{\prime} g$.

Proof. The proof of [41, Proposition 1.2.5] applies. Here we need the existence of the pullbacks and pushouts from Definition 6.1 for the transitivity of the homotopy relation.

After introducing a piece of traditional terminology, we shall state the key theorem about the localization $\mathcal{C}\left[\mathrm{W}^{-1}\right]$ of $\mathcal{C}$.

Definition 6.6. Let $\mathcal{C}$ be a model category and $\mathrm{W}$ the class of weak equivalences. Then the category $\mathcal{C}\left[\mathrm{W}^{-1}\right]$ is called the homotopy category of $\mathcal{C}$ and denoted by Ho $\mathcal{C}$.

Theorem 6.7. Let $\mathcal{C}$ together with (Cof, W, Fib) be a model category, let $Q: \mathcal{C} \rightarrow$ $\mathrm{Ho} \mathcal{C}$ be the canonical localization functor, and denote by $\mathcal{C}_{c f}$ the full subcategory given by the objects which are cofibrant and fibrant.

(1) The composition $\mathcal{C}_{c f} \stackrel{\subseteq}{\longrightarrow} \mathcal{C} \stackrel{Q}{\longrightarrow}$ HoC induces a category equivalence $\left(\mathcal{C}_{c f} / \sim\right) \rightarrow$ Ho $\mathcal{C}$, where $\mathcal{C}_{c f} / \sim$ is defined by $\left(\mathcal{C}_{c f} / \sim\right)(X, Y)=\mathcal{C}_{c f}(X, Y) / \sim$. In particular, $\mathrm{Ho} \mathcal{C}$ is a legal category with small homomorphisms spaces. 
(2) If $X \in \mathcal{C}$ is cofibrant and $Y \in \mathcal{C}$ is fibrant, then $Q$ induces an isomorphism $\mathcal{C}(X, Y) / \sim \stackrel{\cong}{\longrightarrow} \operatorname{Ho} \mathcal{C}(Q X, Q Y)$. In particular, there are canonical isomorphisms $\mathcal{C}(C X, F Y) / \sim \stackrel{\cong}{\longrightarrow} \operatorname{Ho} \mathcal{C}(Q X, Q Y)$ for arbitrary $X, Y \in \mathcal{C}$, where $C X$ is a cofibrant replacement of $X$ and $F Y$ is a fibrant replacement of $Y$.

(3) If $f: X \rightarrow Y$ is a morphism in $\mathcal{C}$, them $Q f$ is an isomorphisms if and only if $f$ is a weak equivalence.

Proof. These are standard facts dating back to 60. We refer to [41, Theorem $1.2 .10]$ or [38, $\S 8.4]$ for a proof.

6.2. Exact model structures. It is no surprise that following [42, we shall focus on exact categories with model structure compatible with the exact structure.

Definition 6.8. Let $\mathcal{E}$ be an exact category. An exact model structure on $\mathcal{E}$ is a model structure (Cof, W, Fib) such that (Cof, W $\cap$ Fib) and (Cof $\cap$ W, Fib) are exact weak factorization systems in the sense of Definition 5.11. An exact model category is a weakly idempotent complete exact category $\mathcal{E}$ together with an exact model structure.

Exact model structures are clearly determined by the classes of cofibrant and fibrant objects $\mathcal{C}$ and $\mathcal{F}$, respectively. Following the same philosophy, we shall consider the class $\mathcal{W}$ of trivial objects instead of the class $\mathrm{W}$ of weak equivalences. Here, and object $X \in \mathcal{E}$ is trivial if $0 \rightarrow X$ is a weak equivalence.

The next theorem is a result due to Hovey 42 which describes what conditions the classes $\mathcal{C}, \mathcal{W}, \mathcal{F}$ must satisfy in order to determine an exact model structure on $\mathcal{E}$. The result was stated in this generality in 31, but we prefer to give a full proof here.

Theorem 6.9. Let $\mathcal{E}$ be a weakly idempotent complete exact category. There is a bijective correspondence between exact model structures on $\mathcal{E}$ and the triples of classes $(\mathcal{C}, \mathcal{W}, \mathcal{F})$ satisfying the following conditions

(1) $\mathcal{W}$ is closed under retracts and satisfies the 2-out-of-3 property for extensions. That is, if $0 \rightarrow X \rightarrow Y \rightarrow Z \rightarrow 0$ is a conflation in $\mathcal{E}$ and two of $X, Y, Z$ are in $\mathcal{W}$, then so is the third.

(2) $(\mathcal{C}, \mathcal{W} \cap \mathcal{F})$ and $(\mathcal{C} \cap \mathcal{W}, \mathcal{F})$ are complete cotorsion pairs in $\mathcal{E}$.

The correspondence assigns to $(\mathcal{C}, \mathcal{W}, \mathcal{F})$ the model structure (Cof, W, Fib) such that

(a) $c \in$ Cof if and only if $c$ is an inflation with cokernel in $\mathcal{C}$,

(b) $w \in \mathrm{W}$ if and only if $w=w_{d} w_{i}$ for an inflation $w_{i}$ with cokernel in $\mathcal{W}$ and a deflation $w_{d}$ with kernel in $\mathcal{W}$,

(c) $f \in$ Fib if and only if $f$ is a deflation with kernel in $\mathcal{F}$. 
The inverse assigns to (Cof, W, Fib) the triple $(\mathcal{C}, \mathcal{W}, \mathcal{F})$ such that $\mathcal{C}$ is the class of cofibrant objects, $\mathcal{W}$ is the class of trivial objects, and $\mathcal{F}$ is the class of fibrant objects.

Remark 6.10. If $\mathcal{E}$ is an efficient exact category (Definition 3.4) and the two cotorsion pairs $(\mathcal{C}, \mathcal{W} \cap \mathcal{F})$ and $(\mathcal{C} \cap \mathcal{W}, \mathcal{F})$ are both of the form $\left(^{\perp}\left(\mathcal{S}^{\perp}\right), \mathcal{S}^{\perp}\right)$ for a set $\mathcal{S}$ as in Theorem 5.16, then the resulting exact model structure is cofibrantly generated in the sense of [38, Definition 11.1.2]. This is a very usual situation thanks to results in [32, 67]. In such a case, approximations with respect to the two cotorsion pairs and the corresponding weak factorization systems from Definition 6.1 are all functorial.

Once we prove the theorem, there is an immediate corollary which poses further restrictions on the possible classes of trivial objects.

Corollary 6.11. Let $\mathcal{E}$ be an exact model category with the exact structure given by $(\mathcal{C}, \mathcal{W}, \mathcal{F})$. Then the class $\mathcal{W}$ of trivial objects is generating and cogenerating in $\mathcal{E}$.

Before proving the theorem, we need to establish three lemmas. The first of them is syntactically very similar to a necessary condition for the calculus of noncommutative fractions, see [26, p. 12, cond. 2.2.c)].

Lemma 6.12. [42, 5.6] Let $\mathcal{E}$ be a weakly idempotent complete exact category and $(\mathcal{C}, \mathcal{W}, \mathcal{F})$ be a triple satisfying (1) and (2) from the statement of Theorem 6.9. Denote $\mathrm{TCof}=\operatorname{Infl}(\mathcal{C} \cap \mathcal{W})$ and $\mathrm{TFib}=\operatorname{Defl}(\mathcal{W} \cap \mathcal{F})$, and suppose we have a pair of composable morphisms $p \in \mathrm{TFib}$ and $i \in \mathrm{TCof}$. Then there is commutative square

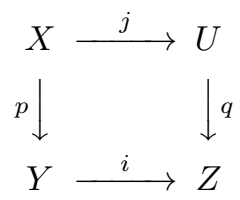

with $j \in$ TCof and $q \in \mathrm{TFib}$.

Proof. If Cof $=\operatorname{Infl} \mathcal{C}$ as in (a) in the theorem, then (Cof, TFib) is an exact weak factorization system by Theorem 5.13 . Consider a factorization of $i p=q j$ of $i p$ with respect to (Cof, TFib). We shall prove that $q, j$ have the desired properties. Using Lemma 3.6. 10, Proposition 3.1] and the exact $3 \times 3$ lemma [10, Corollary 
3.6], we obtain a commutative diagram with conflations in rows and columns

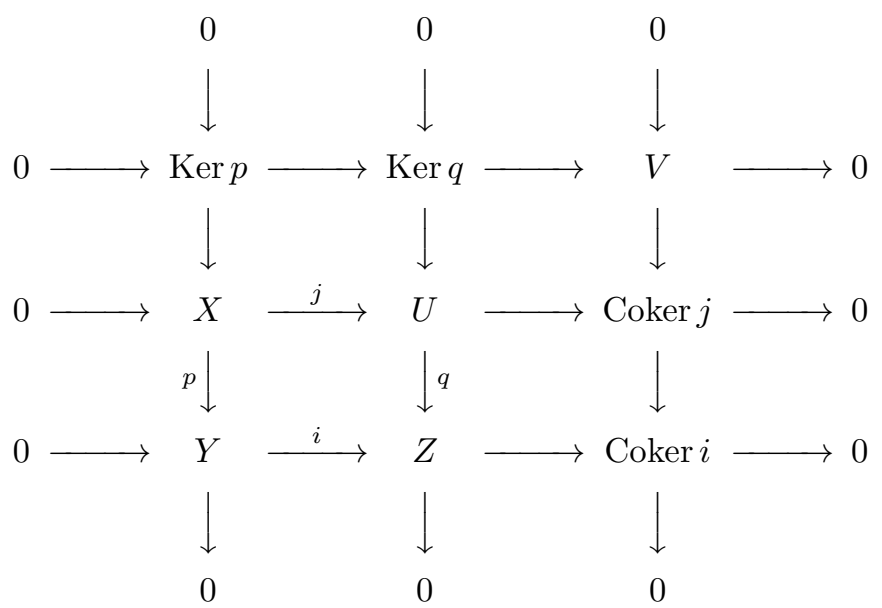

Our task reduces to proving that Coker $j \in \mathcal{W}$. To this end, $V \in \mathcal{W}$ since $\operatorname{Ker} p$, Ker $q \in \mathcal{W}$, and Coker $j \in \mathcal{W}$ since $V$, Coker $i \in \mathcal{W}$.

The second one will be useful for proving the 2-out-of-3 property for $\mathrm{W}$ when we start from $(\mathcal{C}, \mathcal{W}, \mathcal{F})$.

Lemma 6.13. Let $\mathcal{E}$ be a weakly idempotent complete exact category, $(\mathcal{C}, \mathcal{W}, \mathcal{F})$ be a triple satisfying (1) and (2) from Theorem 6.9, and let Cof, TCof, Fib, TFib be as above. Let further $f: X \rightarrow Y$ and $g: Y \rightarrow Z$ be a pair of composable morphisms.

(1) If $g f \in \mathrm{Fib}, f \in \mathrm{Cof}$ and two of the following conditions hold, then so does the third:

(a) $f \in \mathrm{TCof}$,

(b) $g$ is a deflation and Coker $g \in \mathcal{W}$,

(c) $g f \in$ TFib.

(2) If $g f \in \mathrm{Cof}$ and $g \in \mathrm{Fib}$ and two of the following conditions hold, then so does the third:

(a) $f$ is an inflation and $\operatorname{Ker} f \in \mathcal{W}$,

(b) $g \in$ TFib,

(c) $g f \in$ TCof.

Proof. We will prove only (1) as (2) is dual. Our assumption says that $g f$ is a deflation, and so is $g$ by Lemma 3.6. Using Lemma 3.6. again together with 10 , Proposition 2.12], we construct a commutative diagram with conflations in rows 
and columns:

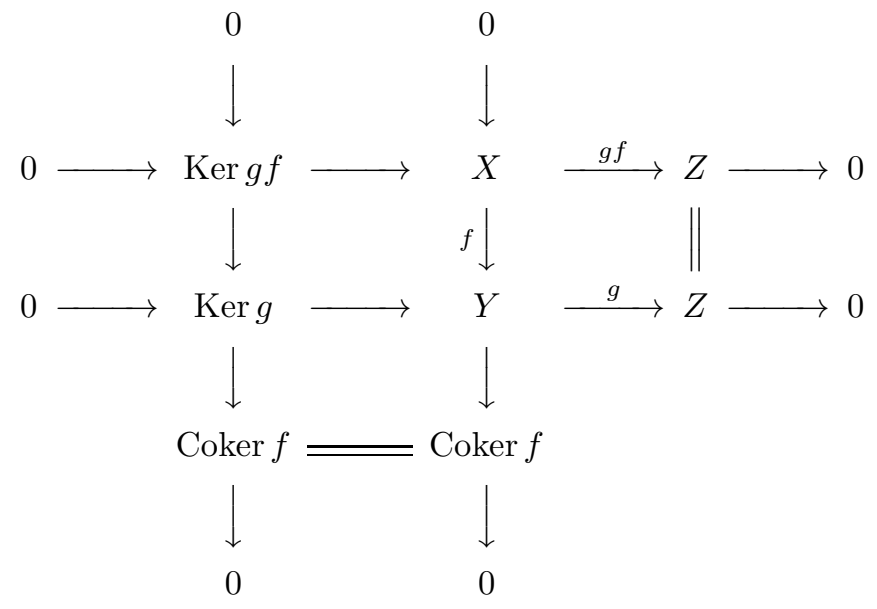

The conclusion follows from the 2-out-of-3 property of $\mathcal{W}$ applied to the leftmost vertical conflation.

The last lemma characterizes weak equivalences among inflations and deflations when starting with an exact model structure (Cof, W, Fib).

Lemma 6.14. Let $\mathcal{E}$ together with (Cof, W, Fib) be an exact model category. If $f$ is an inflation, then $f \in \mathrm{W}$ if and only if Coker $f$ is a trivial object. Dually if $g$ is a deflation, then $g \in \mathrm{W}$ if and only if Ker $g$ is a trivial object.

Proof. We will only prove the statement for inflations, the other case being dual. Let $f$ be an inflation and factor $f$ as $f=p i$ with respect to (Cof, $\mathrm{W} \cap \mathrm{Fib}$ ). Using Lemma 3.6. we get a commutative diagram

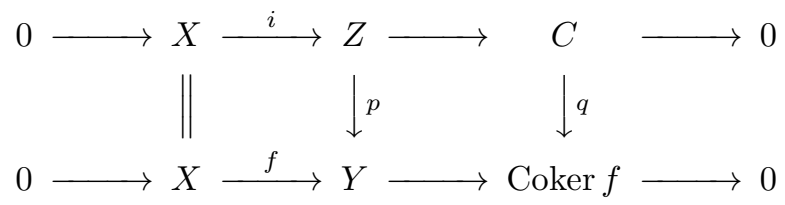

Note that $q$ is a trivial cofibration since the right hand side square is a pushout square by [10, Proposition 2.12]. Now $f$ is a weak equivalence if and only if $i$ is a trivial cofibration if and only if $C$ is trivially cofibrant. Inspecting the composition $C \stackrel{q}{\rightarrow}$ Coker $f \rightarrow 0$, the latter is also equivalent to Coker $f \rightarrow 0$ being a weak equivalence, or in other words, to the fact that Coker $f$ is a trivial object.

Now we can finish the proof of Theorem 6.9.

Proof of Theorem 6.9. Using the notation TCof $=\operatorname{Infl}(\mathcal{C} \cap \mathcal{W})$ and TFib $=\operatorname{Defl}(\mathcal{W} \cap$ $\mathcal{F}$ ), it is clear from Theorem 5.13 that (Cof, TFib) is an exact weak factorization system if and only if $(\mathcal{C}, \mathcal{W} \cap \mathcal{F})$ is a complete cotorsion pair, and similarly for (TCof, Fib) and $(\mathcal{C} \cap \mathcal{W}, \mathcal{F})$. 
Suppose now that $(\mathcal{C}, \mathcal{W}, \mathcal{F})$ is a triple satisfying $(1)$ and $(2)$. Note that the assumptions make sure that $(\mathcal{C} \cap \mathcal{W}, \mathcal{W} \cap \mathcal{F})$ is a complete cotorsion pair in $\mathcal{W}$ when the latter is viewed as an exact category with the exact structure induced from $\mathcal{E}$ (see Proposition 3.1).

Let $\mathrm{W}$ be the class of morphisms as in (b). The same argument as in the proof of Theorem 5.13 shows that all morphisms $w \in \mathrm{W}$ factorize as $w=p i$ with $i \in$ TCof and $p \in$ TFib. Indeed, if $w$ is an inflation with cokernel in $\mathcal{W}$ or a deflation with kernel in $\mathcal{W}$, we construct a diagram similar to $(\ddagger)$ in page 34 with approximation sequences with respect to $(\mathcal{C} \cap \mathcal{W}, \mathcal{W} \cap \mathcal{F})$. If $w=w_{d} w_{i}$, we construct a commutative diagram

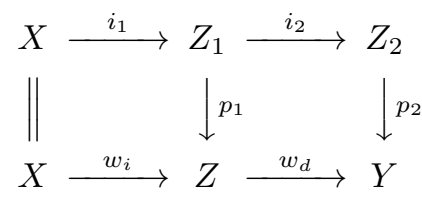

with $i_{1}, i_{2} \in$ TCof and $p_{1}, p_{2}$ is TFib, and we use the fact that $\mathcal{C} \cap \mathcal{W}$ is closed under extensions.

An immediate consequence of the latter description of $\mathrm{W}$ and Lemma 6.13 is that $\mathrm{TCof}=\mathrm{Cof} \cap \mathrm{W}$ and $\mathrm{TFib}=\mathrm{W} \cap \mathrm{Fib}$. This yields the two weak factorization systems required by Definition 6.1(MS2).

It also easily follows that $\mathrm{W}$ is closed under compositions. Indeed, if $i_{1}, i_{2} \in$ TCof and $p_{1}, p_{2} \in$ TFib such that the composition $c=\left(p_{2} i_{2}\right)\left(p_{1} i_{1}\right)$ exists, we can write $c=\left(p_{2} q\right)\left(j i_{1}\right)$ for $j \in$ TCof and $q \in$ TFib by Lemma 6.12

Next we claim that $\mathrm{W}$ has the 2-out-of-3 property for composition. We shall prove that if $w=p i \in \mathrm{W}$ with $i \in \mathrm{TCof}$ and $p \in \mathrm{TFib}$, and if $w f=p i f \in \mathrm{W}$ for some morphism $f$, then $f \in W$. Since we can factor $f$ into a morphism from Cof followed by a morphism from TFib, we can without loss of generality assume that $f \in$ Cof. As $w f \in \mathrm{W}$, we can write $p(i f)=q j$ for some $j \in$ TCof and $q \in$ TFib. If we form the pushout of if $\in$ Cof and $j \in$ TCof, we get a commutative diagram

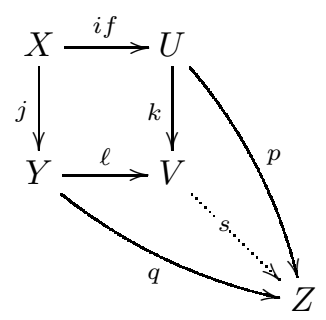

As clearly $k \in$ TCof and $p=s k \in$ TFib, the morphism $s$ is a deflation with Ker $s \in \mathcal{W}$ by Lemma 6.13. As $q=s \ell$, we can apply Lemma 6.13 again and we show that $\ell \in$ TCof. Then clearly if $\in$ TCof as the square in the above diagram is a pushout square. Finally, the conflation

$$
0 \longrightarrow \text { Coker } f \longrightarrow \text { Coker } \text { if } \longrightarrow \text { Coker } i \longrightarrow 0
$$

for the composition if obtained using [10, Lemma 3.5] shows that Coker $f \in \mathcal{W}$. Hence $f \in \mathrm{TCof} \subseteq \mathrm{W}$. The remaining case is dual and we have proved the claim. 
Finally, let $w=w_{1} \oplus w_{2}: X_{1} \oplus X_{2} \rightarrow Y_{1} \oplus Y_{2}$ and suppose $w \in \mathrm{W}$. Then $w_{n}$ factor as $w_{n}=p_{n} i_{n}, i_{n} \in$ Cof, $p_{n} \in \mathrm{TFib}$, for $n=1,2$. Comparing this factorization with the one for $w=p i$ with $i \in$ TCof, $p \in$ TFib for $w$, and using the 2-out-of-3 property for $\mathrm{W}$ proved above, we see that $i_{n} \in \mathrm{TCof}$ for $n=1,2$. Thus $\mathrm{W}$ us closed under retracts, finishing the proof that (Cof, W, Fib) is an exact model structure on $\mathcal{E}$.

Suppose conversely that (Cof, W, Fib) is an exact model structure, and denote by $\mathcal{C}, \mathcal{W}, \mathcal{F}$ the classes of cofibrant, trivial, and fibrant objects, respectively. Then $(\mathcal{C}, \mathcal{W} \cap \mathcal{F})$ and $(\mathcal{C} \cap \mathcal{W}, \mathcal{F})$ are functorially complete cotorsion pairs in $\mathcal{E}$ by Theorem 5.13, As $\mathrm{W}$ is closed under retractions, so is $\mathcal{W}$. In order to prove the 2-out-of-3 property for extensions for $\mathcal{W}$, let

$$
0 \longrightarrow W_{1} \longrightarrow W_{2} \longrightarrow W_{3} \longrightarrow 0
$$

be a conflation. Then if two of $0 \rightarrow W_{1}, W_{1} \rightarrow W_{2}$ and $0 \rightarrow W_{2}$ belong to $\mathrm{W}$, so does the third. If we apply Lemma 6.14, we deduce that if two of $W_{1}, W_{2}, W_{3}$ are trivial objects, so is the third. Hence $(\mathcal{C}, \mathcal{W}, \mathcal{F})$ satisfies Theorem 6.9 (1) and (2), as required.

6.3. Hereditary model structures and triangles. An expected but important point is that the homotopy category of an exact model category often carries a natural algebraic triangulated structure. To this end, recall that a Frobenius exact category is an exact category $\mathcal{E}^{\prime}$ such that

(1) $\mathcal{E}^{\prime}$ has enough projective and injective objects, and

(2) the classes of projective and injective objects coincide.

If $\mathcal{E}^{\prime}$ is such a category, then the quotient $\underline{\mathcal{E}^{\prime}}$ of $\mathcal{E}^{\prime}$ modulo the two-sided ideal of all morphisms which factor through projective injective objects is naturally a triangulated category. We refer to [35, Chapter I] for details. Triangulated categories which arise in this way are called algebraic [48, $\S 7]$.

In order to describe the prospective triangles, we adapt the definition of cofiber sequences from [41, Chapter 6], incorporating the ideas from [35.

Definition 6.15. Let $\mathcal{E}$ together with $(\mathcal{C}, \mathcal{W}, \mathcal{F})$ be an exact model category. A sequence of morphisms

$$
X \stackrel{u}{\longrightarrow} Y \stackrel{v}{\longrightarrow} Z \stackrel{w}{\longrightarrow} \Sigma X
$$

is called a cofiber sequence if $u, v, w$ fit into the following diagram

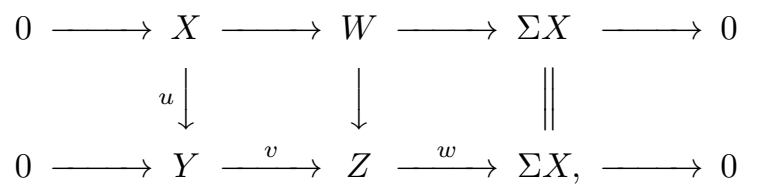

where both rows are conflations in $\mathcal{E}$ and $W \in \mathcal{W}$. 
In order to understand the homotopy category of an exact model category, we must first better understand the homotopy relations. We summarize the essentials in the following lemma.

Lemma 6.16. Let $\mathcal{E}$ be an exact model category and $f, g: X \rightarrow Y$ be two morphisms.

(1) $f$ and $g$ are right homotopic if and only if $f-g$ factors through a trivially cofibrant object.

(2) $f$ and $g$ are left homotopic if and only if $f-g$ factors through a trivially fibrant object.

(3) If $X$ is cofibrant and $Y$ is fibrant, then $f$ and $g$ are homotopic if and only if $f-g$ factors through a trivially fibrant and cofibrant object.

Proof. We refer to [31, Proposition 4.4] or [6, Proposition 1.1.14].

As indicated above, we need another condition in order to construct an algebraic triangulated structure - we need our model structure to be hereditary. The significance of this notion in connection to exact model structures has been very well demonstrated in the work of Gillespie [27, 28, 29, 30, 31. The property we are interested in is described by the following lemma.

Lemma 6.17. Let $\mathcal{E}$ be a weakly idempotent complete exact category and $(\mathcal{A}, \mathcal{B})$ be a cotorsion pair in $\mathcal{E}$ such that $\mathcal{A}$ is generating and $\mathcal{B}$ is cogenerating (e.g. if $(\mathcal{A}, \mathcal{B})$ is complete). Then the following are equivalent:

(1) $\operatorname{Ext}_{\mathcal{E}}^{n}(A, B)=0$ for each $A \in \mathcal{A}, B \in \mathcal{B}$ and $n \geq 1$.

(2) $\operatorname{Ext}_{\mathcal{E}}^{2}(A, B)=0$ for each $A \in \mathcal{A}, B \in \mathcal{B}$.

(3) If $f: A_{1} \rightarrow A_{2}$ is a deflation with $A_{1}, A_{2} \in \mathcal{A}$, then $\operatorname{Ker} f \in \mathcal{A}$.

(4) If $g: B_{1} \rightarrow B_{2}$ is an inflation with $B_{1}, B_{2} \in \mathcal{B}$, then Coker $g \in \mathcal{B}$.

Proof. Essentially the same proof as in [65, Lemma 4.25] applies and the straightforward modifications for our setting can be made using results in [10, $\S 7]$. Alternatively, a different proof for $(\mathcal{A}, \mathcal{B})$ complete was given in [6, Proposition 1.1.12].

The crucial implications are $(3) \Longrightarrow(1)$ and $(4) \Longrightarrow(1)$. The other being dual, it suffices to prove the first one. The key point is that using an argument very similar to [36, Lemma I.4.6(i)] we construct for any $n$-fold extension $\varepsilon: 0 \rightarrow$ $B \rightarrow E_{n} \rightarrow \cdots \rightarrow E_{1} \rightarrow A \rightarrow 0$ a commutative diagram

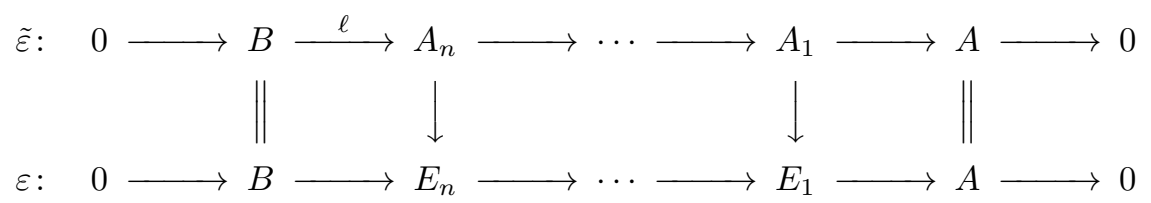

with $A_{i} \in \mathcal{A}$ for $i=1, \ldots, n-1$ and deflations in all columns. Since $\tilde{\varepsilon}$ clearly represents the same element of $\operatorname{Ext}_{\mathcal{E}}^{n}(A, B)$ as $\varepsilon$ and the kernel of $A_{i} \rightarrow A_{i-1}$ 
belongs to $\mathcal{A}$ by (3) for every $1 \leq i \leq n-1$ (here $A_{0}=A$ by convention), we deduce that $\ell: B \rightarrow A_{n}$ splits and $\tilde{\varepsilon}$ represents the zero element.

Definition 6.18. Let $\mathcal{E}$ be a weakly idempotent complete exact category. A hereditary cotorsion pair is a cotorsion pair $(\mathcal{A}, \mathcal{B})$ in $\mathcal{E}$ satisfying the equivalent conditions of Lemma 6.17 (including that $\mathcal{A}$ is generating and $\mathcal{B}$ cogenerating).

Definition 6.19. Let $\mathcal{E}$ be a weakly idempotent complete exact category and let $(\mathcal{C}, \mathcal{W}, \mathcal{F})$ be a triple specifying an exact model structure as in Theorem 6.9. The model structure is called hereditary if the cotorsion pairs $(\mathcal{C}, \mathcal{W} \cap \mathcal{F})$ and $(\mathcal{C} \cap \mathcal{W}, \mathcal{F})$ are hereditary.

A hereditary model category is an exact model category with a hereditary model structure.

One of the key points about hereditary complete cotorsion pairs is that the following version of the Horseshoe Lemma holds:

Lemma 6.20. Let $\mathcal{E}$ be a weakly idempotent complete exact category and $(\mathcal{A}, \mathcal{B})$ be a complete hereditary cotorsion pair. Suppose we have the solid part of the commutative diagram

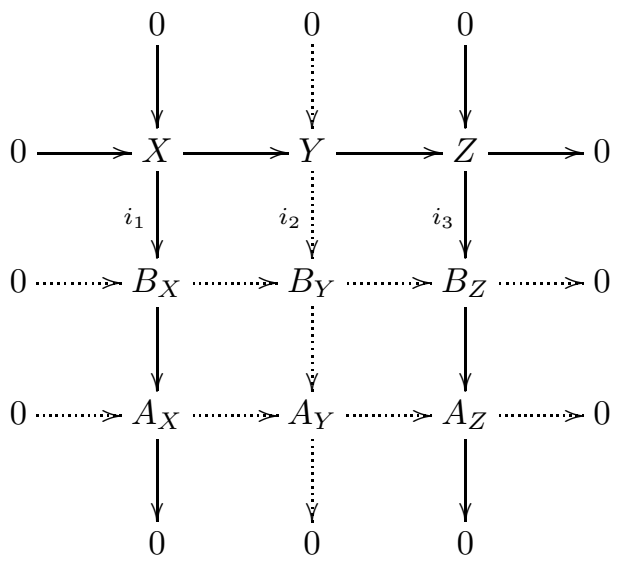

with a conflation in the upper row and approximation sequences in the two columns. Then the dotted part can be completed so that all rows are conflations and all columns are approximation sequences.

Proof. A proof can be found for instance in [32, Lemma 5.2.3] and other references in [71, Lemma 3.3]. We construct the pushout

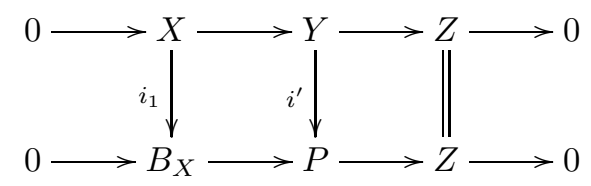


Since $\operatorname{Ext}_{\mathcal{E}}^{2}\left(A_{Z}, B_{X}\right)=0$, we can use the long exact sequence of Ext's and construct the commutative diagram

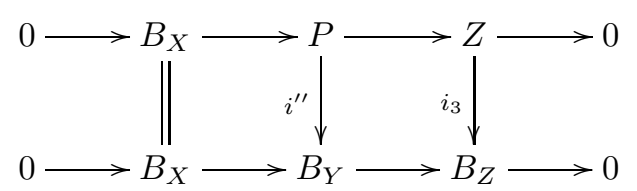

Finally we simply put $i_{2}=i^{\prime \prime} i^{\prime}$.

Now we are in a position to state and prove the main result of the section.

Theorem 6.21. Let $\mathcal{E}$ be a hereditary model category, the exact model structure given by $(\mathcal{C}, \mathcal{W}, \mathcal{F})$, and denote $\mathcal{E}_{c f}=\mathcal{C} \cap \mathcal{F}$ and $\omega=\mathcal{E}_{c f} \cap \mathcal{W}$.

(1) $\mathcal{E}_{c f}$ with the induced exact structure is a Frobenius exact category and $\omega$ is precisely the class of projective injective objects in $\mathcal{E}_{c f}$. In particular, Ho $\mathcal{E}$ is an algebraic triangulated category.

(2) Every cofiber sequence in $\mathcal{E}$ becomes a triangle in $\mathrm{Ho}_{\mathcal{E}}$, and conversely every triangle is isomorphic in $\mathrm{Ho} \mathcal{E}$ to a cofiber sequence. In particular, every conflation $0 \rightarrow X \stackrel{u}{\rightarrow} Y \stackrel{v}{\rightarrow} Z \rightarrow 0$ yields a triangle in Ho $\mathcal{E}$ :

$$
X \stackrel{u}{\longrightarrow} Y \stackrel{v}{\longrightarrow} Z \stackrel{w}{\longrightarrow} \Sigma X \text {. }
$$

Proof. Part (1) is an easy consequence of Lemmas 6.16 and 6.17 and it is proved in 31, Proposition 5.2]; see also 6, Proposition 1.1.15].

We focus on (2) and suppose that we have a cofiber sequence given by the diagram

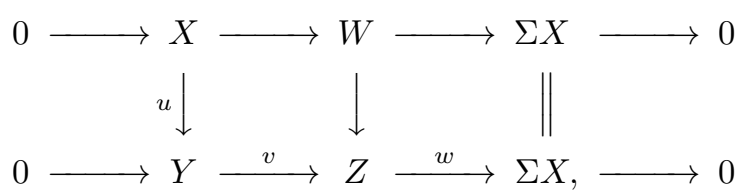

with $W \in \mathcal{W}$. If we choose approximation sequences of $X, Y$ and $\Sigma X$ with respect to the cotorsion pair $(\mathcal{C} \cap \mathcal{W}, \mathcal{F})$, we can use Lemma 6.20 to construct the following commutative diagram

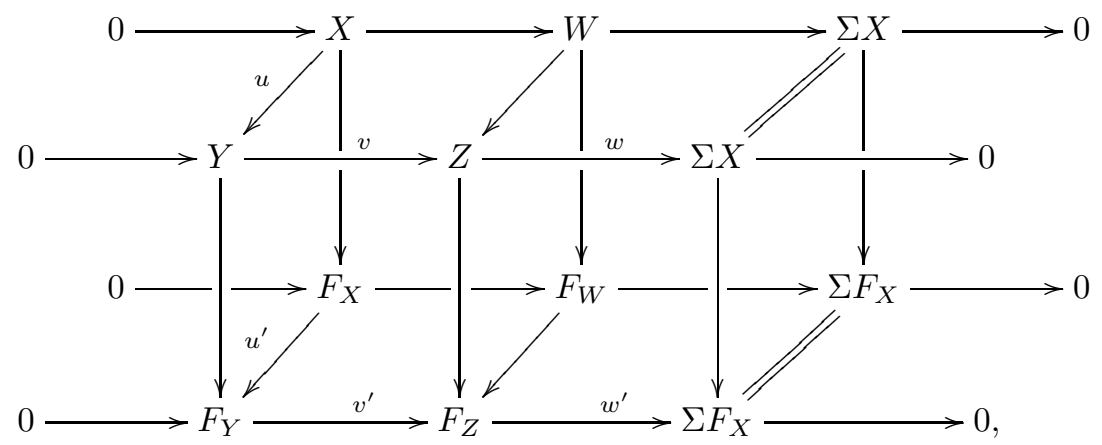


where all rows are conflations, all objects at the bottom are fibrant, and all vertical morphisms are trivial cofibrations. In particular,

$$
F_{X} \stackrel{u^{\prime}}{\longrightarrow} F_{Y} \stackrel{v^{\prime}}{\longrightarrow} F_{Z} \stackrel{w^{\prime}}{\longrightarrow} \Sigma F_{X}
$$

is a cofiber sequence of fibrant objects and it is isomorphic in Ho $\mathcal{E}$ to the original one. In a similar way, we can find a cofiber sequence in $\mathcal{E}_{c f}$ which is isomorphic to the original one in Ho $\mathcal{E}$. But the latter one is necessarily a triangle by [35, I.2.5, p. 15-16].

The last statement is clear since $\mathcal{W}$ is generating, and thus every short exact sequence $0 \rightarrow Y \stackrel{v}{\rightarrow} Z \stackrel{w}{\rightarrow} \Sigma X \rightarrow 0$ is a part of a diagram $(*)$ which defines a cofiber sequence.

\section{Models for the derived category}

In this section we will be concerned with the construction of models for the unbounded derived category of an exact category of Grothendieck type (Definition 3.11). We will show that there always exists at least one model structure (the one induced by injectives) if the exact category $\mathcal{E}$ arises as in Theorem 3.16] There is usually a lot of model structures for the derived category of a Grothendieck category. A recipe for their construction has been found by Gillespie [27, 28, 29] and various versions of the result were discussed in [23, 65, but our presentation here is based on an elegant idea from [71. Finally, we will discuss probably the most interesting example available so far which is essentially due to Neeman: the projective model structure on the derived category of flat modules.

The guiding principle for our constructions is the following consequence of Theorem 6.9 and Corollary 6.11.

Proposition 7.1. Let $\mathcal{E}$ be a weakly idempotent complete exact category and $\mathcal{W} \subseteq$ $\mathcal{E}$ be a generating and cogenerating class of objects which is closed under retracts in $\mathcal{E}$ and satisfies the 2-out-of-3 property for extensions.

If $\left(\mathcal{A}_{0}, \mathcal{B}_{0}\right)$ is a complete hereditary cotorsion pair in $\mathcal{W}$ such that there exist complete cotorsion pairs $\left(\mathcal{C}, \mathcal{B}_{0}\right)$ and $\left(\mathcal{A}_{0}, \mathcal{F}\right)$ in $\mathcal{E}$, then $\mathcal{E}$ together with $(\mathcal{C}, \mathcal{W}, \mathcal{F})$ is a hereditary model category.

Proof. This is a direct consequence of Theorem 6.9 and Lemma 6.17

7.1. Lifting cotorsion pairs to categories of complexes. Let us briefly discuss our setting and the tools to achieve the above goals. We start with an exact category $\mathcal{E}$ of Grothendieck type and consider the category $\mathbf{C}(\mathcal{E})$ of cochain complexes over $\mathcal{E}$ with the natural exact structure: A sequence $0 \rightarrow X \rightarrow Y \rightarrow Z \rightarrow 0$ of complexes is a conflation precisely when all the component sequences $0 \rightarrow X^{n} \rightarrow$ $Y^{n} \rightarrow Z^{n} \rightarrow 0, n \in \mathbb{Z}$, are conflations.

As we are interested in the derived category $\mathbf{D}(\mathcal{E})$, our class of trivial objects will be $\mathcal{W}=\mathbf{C}_{\mathrm{ac}}(\mathcal{E})$, the class of all acyclic complexes. Here, we call a complex

$$
X: \quad \cdots \longrightarrow X^{0} \longrightarrow X^{1} \longrightarrow X^{2} \longrightarrow X^{3} \longrightarrow \cdots
$$


acyclic (or exact) if it arises by splicing countably many conflations in $\mathcal{E}$ of the form

$$
0 \longrightarrow Z^{i} \longrightarrow X^{i} \longrightarrow Z^{i+1} \longrightarrow 0 .
$$

In order to justify that this is a valid choice for $\mathcal{W}$, we need to prove that the necessary conditions from Theorem 6.9 and Corollary 6.11 are satisfied. To this end, we need a definition and a lemma.

Definition 7.2. [10, 8.1 and 8.8] Let $\mathcal{E}$ be an exact category. A morphism $f: X \rightarrow$ $Y$ is called admissible if it can be written as a composition $f=i d$, where $i$ is an inflation and $d$ is a deflation. A sequence $X \stackrel{f}{\rightarrow} Y \stackrel{f^{\prime}}{\rightarrow} Z$ of admissible morphisms $f=i d$ and $f^{\prime}=i^{\prime} d^{\prime}$ is exact if $0 \rightarrow I \stackrel{i}{\rightarrow} Y \stackrel{d^{\prime}}{\rightarrow} I^{\prime} \rightarrow 0$ is a conflation.

In other words, admissible morphisms are precisely those for which it makes sense to speak of an image. If $\mathcal{E}$ is abelian, every morphism is admissible.

Lemma 7.3. Let $\mathcal{E}$ be an exact category and suppose that we have a commutative diagram

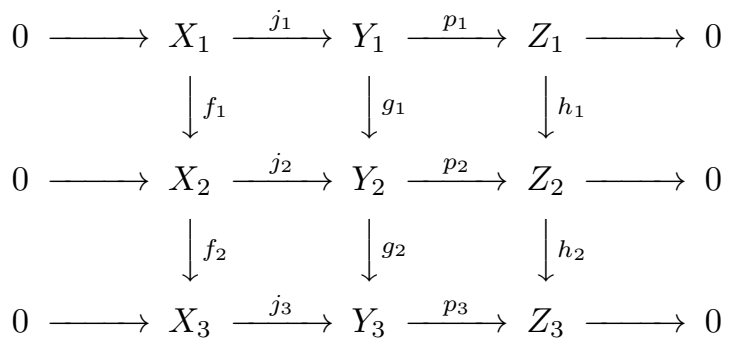

with conflations in rows. If $f_{2}, h_{1}, h_{2}$ are admissible, $g_{2} g_{1}=0$ and $Z_{1} \stackrel{h_{1}}{\rightarrow} Z_{2} \stackrel{h_{2}}{\rightarrow} Z_{3}$ is exact, then $g_{2}$ is admissible and we have a commutative diagram.

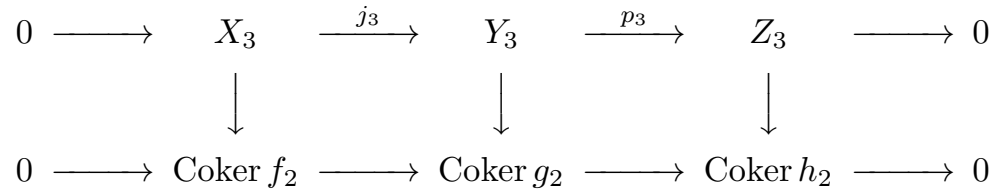

with conflations in rows and cokernel homomorphisms in columns.

Proof. If we take the pushout of the lower conflation along $X_{3} \rightarrow$ Coker $f_{2}$, we get a diagram of the form

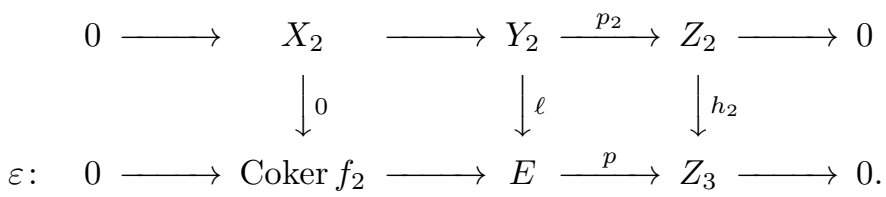

An easy diagram chase reveals that there exists $k: Z_{2} \rightarrow E$ such that $\ell=k p_{2}$ and $h_{2}=p k$. Since $0=\ell g_{1}=k p_{2} g_{1}=k h_{1} p_{1}$ and $p_{1}$ is an epimorphism, we get 
$k h_{1}=0$. Hence $k$ factors through the image of $h_{2}$, and if we denote the inflation $\operatorname{Im} h_{2} \rightarrow Z_{3}$ by $i$, we obtain a morphisms $k^{\prime}: \operatorname{Im} h_{2} \rightarrow Y_{3}$ such that $i=p k^{\prime}$. Considering the equivalence class $[\varepsilon] \in \operatorname{Ext}_{\mathcal{E}}^{1}\left(Z_{3}\right.$, Coker $\left.f_{2}\right)$, the existence of $k^{\prime}$ just says that

$$
\operatorname{Ext}_{\mathcal{E}}^{1}\left(i, \text { Coker } f_{2}\right)([\varepsilon])=0 .
$$

Using the long exact sequence of Ext groups, this precisely means that there is a commutative diagram

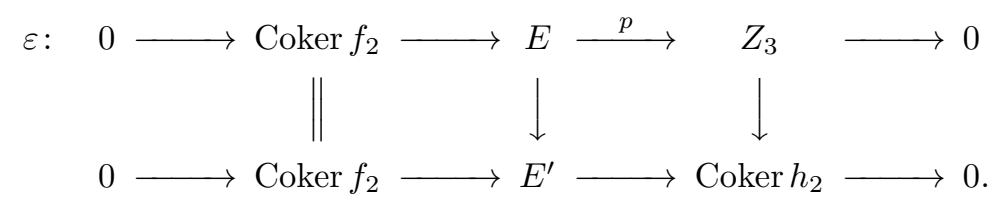

It follows from the construction that the composition $Y_{3} \rightarrow E \rightarrow E^{\prime}$ is a deflation and it is an easy application of [10, Corollaries 3.2 and 3.6] that $g_{2}$ is admissible and the morphism $Y_{3} \rightarrow E^{\prime}$ is a cokernel of $g_{2}$.

Now we can justify that the class $\mathcal{W}=\mathbf{C}_{\text {ac }}(\mathcal{E})$ is a well chosen class of trivial objects for an exact model structure. We also introduce some notation which will be useful later.

Notation 7.4. Let $\mathcal{E}$ be an additive category, $X \in \mathcal{E}$ an object and $n$ an integer. We denote by $D^{n}(X)$ the complex

$$
D^{n}(X): \quad \cdots \longrightarrow 0 \longrightarrow X \stackrel{1_{X}}{\longrightarrow} X \longrightarrow 0 \longrightarrow \cdots
$$

concentrated in cohomological degrees $n$ and $n+1$, and by $S^{n}(X)$ the complex

$$
S^{n}(X): \quad \cdots \longrightarrow 0 \longrightarrow X \longrightarrow 0 \longrightarrow 0 \longrightarrow \cdots
$$

concentrated in cohomological degree $n$.

Proposition 7.5. Let $\mathcal{E}$ be a weakly idempotent complete exact category and consider $\mathbf{C}(\mathcal{E})$ with the exact structure induced from $\mathcal{E}$ (i.e. conflations in $\mathbf{C}(\mathcal{E})$ are defined as component wise conflations). Then $\mathcal{W}=\mathbf{C}_{\mathrm{ac}}(\mathcal{E})$ is generating and cogenerating in $\mathbf{C}(\mathcal{E})$, it is closed under retracts and has the 2-out-of-3 property for extensions.

Proof. The fact that $\mathcal{W}$ is generating and cogenerating in $\mathbf{C}(\mathcal{E})$ is easily seen by the adjunctions

$$
\mathcal{E}\left(X, Y^{n}\right) \cong \mathbf{C}(\mathcal{E})\left(D^{n}(X), Y\right) \quad \text { and } \quad \mathcal{E}\left(Y^{n}, X\right) \cong \mathbf{C}(\mathcal{E})\left(Y, D^{n-1}(X)\right)
$$

for each $X \in \mathcal{E}$ and $Y \in \mathbf{C}(\mathcal{E})$. The 2-out-of-3 property for extensions follows by Lemma 7.3 and the $3 \times 3$ Lemma [10, Corollary 3.6]. The closure of $\mathcal{W}$ under retracts is clear. 
In order to apply Proposition 7.1, we first need to construct complete hereditary cotorsion pairs in $\mathcal{W}$. In that respect, we show that every complete hereditary cotorsion pair $(\mathcal{A}, \mathcal{B})$ in $\mathcal{E}$ can be lifted to a complete hereditary cotorsion pair in $\mathcal{W}$. Note that there are also alternative ways to lift cotorsion pairs to categories of complexes, see e.g. [30] or [21, Chapter 7]. Before stating the result, we again introduce some notation.

Notation 7.6. Let $\mathcal{E}$ be an exact category and $\mathcal{A} \subseteq \mathcal{E}$ be extension closed. Then $\mathcal{A}$ is an exact category by Proposition 3.1 and $\mathbf{C}_{\mathrm{ac}}(\mathcal{A})$ can be viewed as a full subcategory of $\mathbf{C}(\mathcal{E})$. In this context, we denote for brevity $\mathbf{C}_{\mathrm{ac}}(\mathcal{A})$ by $\tilde{\mathcal{A}}$ (this notation has been used by Gillespie in [27, 28, 29]).

Proposition 7.7. Let $\mathcal{E}$ be a weakly idempotent complete exact category and consider $\mathcal{W}=\mathbf{C}_{\mathrm{ac}}(\mathcal{E})$ with the exact structure induced from $\mathcal{E}$. If $(\mathcal{A}, \mathcal{B})$ is a complete hereditary cotorsion pair in $\mathcal{E}$, then $(\tilde{\mathcal{A}}, \tilde{\mathcal{B}})$ is a complete hereditary cotorsion pair in $\mathcal{W}$.

Proof. We first claim that $\operatorname{Ext}_{\mathcal{W}}^{1}(A, B)=0$ for all $A \in \tilde{\mathcal{A}}$ and $B \in \tilde{\mathcal{B}}$. Using the trick from [21, §7.3], we write every $A \in \tilde{\mathcal{A}}$ as an extension

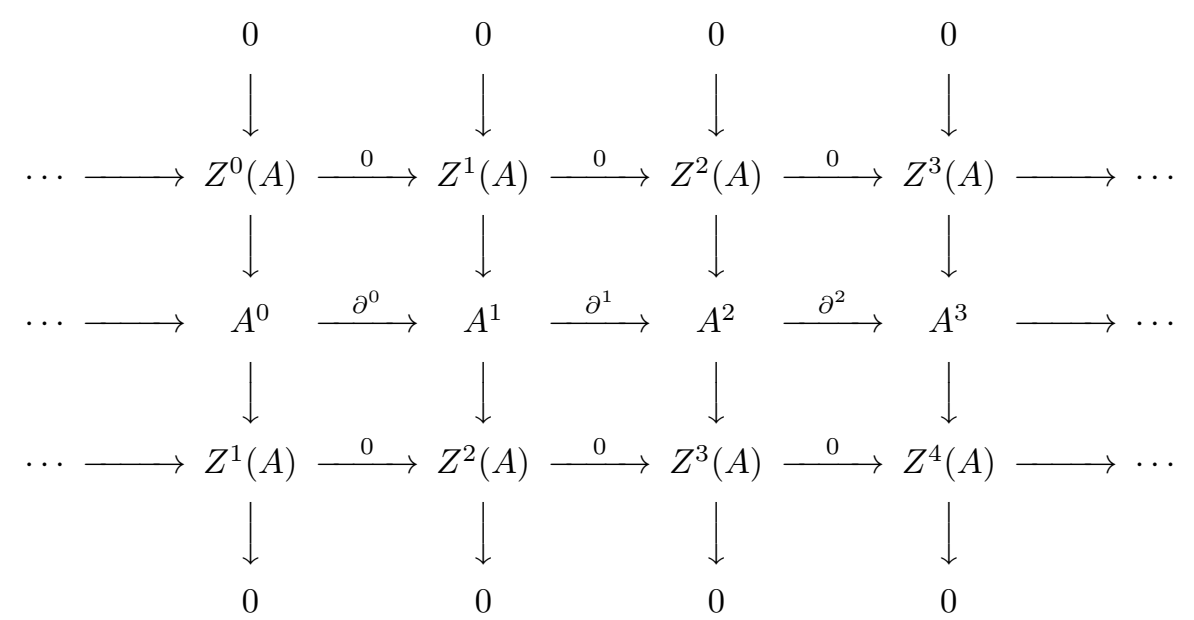

in $\mathbf{C}(\mathcal{E})$, where $Z^{n}(A)=\operatorname{Ker} \partial^{n}$ for $n \in \mathbb{Z}$. Hence it suffices to prove that $\operatorname{Ext}_{\mathbf{C}(\mathcal{E})}^{1}\left(S^{n}\left(A^{\prime}\right), B\right)=0$ for every $A^{\prime} \in \mathcal{A}$ and $B \in \tilde{\mathcal{B}}$ (see Notation 7.4). As any extension of $B$ by $S^{n}\left(A^{\prime}\right)$ is necessarily component wise split, it is enough to show that every cochain complex map $S^{n}\left(A^{\prime}\right) \rightarrow B$ is null-homotopic, but this is clear from $\operatorname{Ext}_{\mathcal{E}}^{1}\left(A^{\prime}, Z^{n-1}(B)\right)=0$. This proves the claim.

Clearly $\tilde{\mathcal{A}}$ and $\tilde{\mathcal{B}}$ are closed under retracts since $\mathcal{A}$ and $\mathcal{B}$ are. Moreover, if $X \in \mathcal{W}$ and we fix approximation sequences $0 \rightarrow Z^{n}(X) \rightarrow B_{n}^{\prime} \rightarrow A_{n}^{\prime} \rightarrow 0$ and $0 \rightarrow B_{n}^{\prime \prime} \rightarrow A_{n}^{\prime \prime} \rightarrow Z^{n}(X) \rightarrow 0$ in $\mathcal{E}$ with respect to $(\mathcal{A}, \mathcal{B})$ for each $n \in \mathbb{Z}$, then Lemma 6.20 allows us to construct approximation sequences of $X$ in $\mathcal{W}$ with respect to $(\tilde{\mathcal{A}}, \tilde{\mathcal{B}})$ (this trick appeared in $[71])$. It follows that $(\tilde{\mathcal{A}}, \tilde{\mathcal{B}})$ is a complete cotorsion pair in $\mathcal{W}$.

Finally, the fact that $(\tilde{\mathcal{A}}, \tilde{\mathcal{B}})$ is hereditary is a rather easy consequence of Lemma 6.17 
Remark 7.8. For the sake of completeness we note that if $\mathcal{E}$ is deconstructible and closed under retracts in a Grothendieck category (hence exact of Grothendieck type by Theorem (3.16), and if $\mathcal{A}$ is deconstructible in $\mathcal{E}$, then $\tilde{\mathcal{A}}$ is deconstructible in $\mathcal{W}=\mathbf{C}_{\mathrm{ac}}(\mathcal{E})$. This follows from Lemma 3.18 and [67, Proposition 4.4]. In such a case, $(\tilde{\mathcal{A}}, \tilde{\mathcal{B}})$ is even functorially complete in $\mathcal{W}$ by Theorem 5.16

The final task in constructing a hereditary model structure using Proposition 7.1 consists in making good choices for the cotorsion pair $(\mathcal{A}, \mathcal{B})$ in $\mathcal{E}$ so that $\left({ }^{\perp} \tilde{\mathcal{B}}, \tilde{\mathcal{B}}\right)$ and $\left(\tilde{\mathcal{A}}, \tilde{\mathcal{A}}^{\perp}\right)$ are complete cotorsion pairs in $\mathbf{C}(\mathcal{E})$. There does not seem to be any known method for proving that $\left({ }^{\perp} \tilde{\mathcal{B}}, \tilde{\mathcal{B}}\right)$ is a complete cotorsion pair at the level of generality we have worked in so far. It is, however, possible to show this in various still very general situations, and this is what we are going to discuss in the rest of this section.

7.2. The injective model structure. First we are going to prove that for all known examples of exact categories $\mathcal{E}$ of Grothendieck type we have a so-called injective model structure for the unbounded derived category $\mathbf{D}(\mathcal{E})$. As indicated, we need an additional mild assumption on $\mathcal{E}$, namely that $\mathbf{C}_{\mathrm{ac}}(\mathcal{E})$ is deconstructible in $\mathbf{C}(\mathcal{E})$. This is true for all exact categories of Grothendieck type which arise as in Theorem 3.16 .

Lemma 7.9. Let $\mathcal{E}$ be a full subcategory of a Grothendieck category which is deconstructible and closed under retracts, considered with the induced exact structure (hence $\mathcal{E}$ is exact of Grothendieck type by Theorem [3.16). Then $\mathbf{C}_{\mathrm{ac}}(\mathcal{E})$ is deconstructible in $\mathbf{C}(\mathcal{E})$.

Proof. This is a special case of [67, Proposition 4.4], since $\mathbf{C}_{\mathrm{ac}}(\mathcal{E})=\tilde{\mathcal{E}}$ in Notation [7.6.

Under this additional assumption we can show that $\left(\tilde{\mathcal{A}}, \tilde{\mathcal{A}}^{\perp}\right)$ from the previous section is always a complete cotorsion pair. The lemma will be also useful later.

Lemma 7.10. [71, 3.5] Let $\mathcal{E}$ be an exact category of Grothendieck type such that $\mathbf{C}_{\mathrm{ac}}(\mathcal{E})$ is deconstructible in $\mathbf{C}(\mathcal{E})$. If $(\mathcal{A}, \mathcal{B})$ is a complete hereditary cotorsion pair in $\mathcal{E}$, then $\left(\tilde{\mathcal{A}}, \tilde{\mathcal{A}}^{\perp}\right)$ is a complete (and hereditary) cotorsion pair in $\mathbf{C}(\mathcal{E})$.

Proof. The special case for $\mathcal{A}=\mathcal{E}$ follows from Theorem [5.16. Suppose that $(\mathcal{A}, \mathcal{B})$ is an arbitrary complete hereditary cotorsion pair in $\mathcal{E}$. Given a complex $X \in \mathbf{C}(\mathcal{E})$, we must construct approximation sequences with respect to $\left(\tilde{\mathcal{A}}, \tilde{\mathcal{A}}^{\perp}\right)$. To this end, consider an approximation sequence $0 \rightarrow X \rightarrow I \rightarrow E \rightarrow 0$ with respect to $\left(\tilde{\mathcal{E}}, \tilde{\mathcal{E}}^{\perp}\right)$, i.e. $E \in \tilde{\mathcal{E}}=\mathbf{C}_{\mathrm{ac}}(\mathcal{E})$ and $I \in \tilde{\mathcal{E}}^{\perp}$. Using Proposition 7.7 we obtain an approximation sequence $0 \rightarrow B^{E} \rightarrow A^{E} \rightarrow E \rightarrow 0$ in $\mathbf{C}_{\mathrm{ac}}(\mathcal{E})$ with respect to $(\tilde{\mathcal{A}}, \tilde{\mathcal{B}})$. Then we find an approximation sequence for $X$ in $\mathbf{C}(\mathcal{E})$ with 
respect to $\left(\tilde{\mathcal{A}}, \tilde{\mathcal{A}}^{\perp}\right)$ in the second row of the following pullback diagram

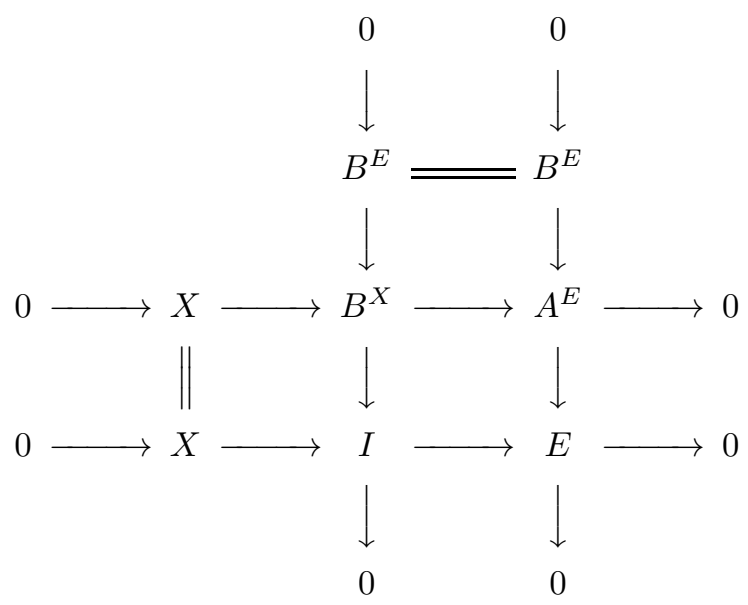

The other approximation sequence for $X$ is obtained analogously by taking an approximation sequence $0 \rightarrow I^{\prime} \stackrel{f}{\rightarrow} E^{\prime} \rightarrow X \rightarrow 0$ with $E^{\prime} \in \tilde{\mathcal{E}}$ and $I^{\prime} \in \tilde{\mathcal{E}}^{\perp}$, and considering the pullback of an approximation sequence $0 \rightarrow B^{E^{\prime}} \rightarrow A^{E^{\prime}} \rightarrow E^{\prime} \rightarrow 0$ along $f$ (see the proof of [71, Theorem 3.5] for details).

Now we can prove the existence of a model structure for $\mathbf{D}(\mathcal{E})$.

Theorem 7.11. Let $\mathcal{E}$ be an exact category of Grothendieck type (Definition 3.11) such that $\mathbf{C}_{\mathrm{ac}}(\mathcal{E})$ is deconstructible in $\mathbf{C}(\mathcal{E})$. Then there is a hereditary model structure (Definition 6.19) on $\mathbf{C}(\mathcal{E})$ such that

(1) Every object of $\mathbf{C}(\mathcal{E})$ is cofibrant.

(2) The trivial objects are precisely the acyclic complexes, $\mathcal{W}=\mathbf{C}_{\mathrm{ac}}(\mathcal{E})$.

(3) The class of fibrant objects is $\mathcal{F}=\mathbf{C}_{\mathrm{ac}}(\mathcal{E})^{\perp}$.

In particular we have $\mathrm{Ho} \mathbf{C}(\mathcal{E})=\mathbf{D}(\mathcal{E})$, the unbounded derived category of $\mathcal{E}$.

Proof. We start with the trivial cotorsion pair $(\mathcal{E}, \mathcal{I})$ in $\mathcal{E}$, whose existence is guaranteed by Corollary 5.9 and which clearly is hereditary. Then we lift it to a complete cotorsion pair $(\tilde{\mathcal{E}}, \tilde{\mathcal{I}})$ in $\mathbf{C}_{\text {ac }}(\mathcal{E})$ - this is in fact an overkill since $\tilde{\mathcal{I}}=\operatorname{Inj} \mathbf{C}(\mathcal{E})$. Then clearly $(\mathbf{C}(\mathcal{E}), \tilde{\mathcal{I}})$ is a complete cotorsion pair (the trivial one) and $\left(\tilde{\mathcal{E}}, \tilde{\mathcal{E}}^{\perp}\right)$ is complete by Lemma 7.10

Thus, we can apply Proposition 7.1 to obtain a hereditary model structure on $\mathbf{C}(\mathcal{E})$ determined by $(\mathcal{C}, \mathcal{W}, \mathcal{F})=\left(\mathbf{C}(\mathcal{E}), \mathbf{C}_{\mathrm{ac}}(\mathcal{E}), \tilde{\mathcal{E}}^{\perp}\right)$ (see Theorem 6.9) . Note that the weak equivalences are the quasi-isomorphisms in the usual sense, that is the morphisms whose mapping cone is acyclic. To see that, note that the usual mapping cone construction is a special case of a cofiber sequence from Definition 6.15 and that $f: X \rightarrow Y$ is a weak equivalence if and only if the third object in some (or any) cofiber sequence is trivial by Lemma 6.14. Hence $\operatorname{Ho} \mathbf{C}(\mathcal{E})=\mathbf{D}(\mathcal{E})$. 
7.3. Models for Grothendieck categories. If $\mathcal{G}$ is a Grothendieck category, we can use any complete hereditary cotorsion pair to construct a model structure for $\mathbf{D}(\mathcal{G})$. This freedom in the construction is very useful when attempting to construct monoidal model structures as we shall show in Section 8, Various weaker versions of this result have been previously proved in [23, 27, 28, 29, 65, 71].

Let us explain how the construction works. First of all, we will use the following simple adjunction formulas for Ext.

Lemma 7.12. [30, 4.2] Let $\mathcal{G}$ be an abelian category, $X \in \mathcal{G}$ be an object and $Y \in \mathbf{C}(\mathcal{G})$ a complex. Then there are natural monomorphisms

$$
\operatorname{Ext}_{\mathcal{G}}^{1}\left(X, Z^{n}(Y)\right) \longrightarrow \operatorname{Ext}_{\mathbf{C}(\mathcal{G})}^{1}\left(S^{n}(X), Y\right)
$$

and

$$
\operatorname{Ext}_{\mathcal{G}}^{1}\left(Y^{n} / B^{n}(Y), X\right) \longrightarrow \operatorname{Ext}_{\mathbf{C}(\mathcal{G})}^{1}\left(Y, S^{n}(X)\right),
$$

where $Z^{n}(Y)=\operatorname{Ker}\left(Y^{n} \rightarrow Y^{n+1}\right)$ and $B^{n}(Y)=\operatorname{Im}\left(Y^{n-1} \rightarrow Y^{n}\right)$ are the usual $n$-th cocycle and coboundary objects of $Y$, respectively. If, moreover, $Y \in \mathbf{C}_{\mathrm{ac}}(\mathcal{G})$ is an acyclic complex, then these are isomorphisms.

Given a complete hereditary cotorsion pair $(\mathcal{A}, \mathcal{B})$ in $\mathcal{G}$, this allows us to deduce the existence of cotorsion pairs $\left({ }^{\perp} \tilde{\mathcal{B}}, \tilde{\mathcal{B}}\right)$ and $\left(\tilde{\mathcal{A}}, \tilde{\mathcal{A}}^{\perp}\right)$ in $\mathbf{C}(\mathcal{G})$.

Lemma 7.13. Let $\mathcal{G}$ be an abelian category and $(\mathcal{A}, \mathcal{B})$ be a cotorsion pair in $\mathcal{G}$ such that $\mathcal{A}$ is generating and $\mathcal{B}$ is cogenerating (e.g. if $(\mathcal{A}, \mathcal{B})$ is complete). Then

$$
\tilde{\mathcal{B}}=\left\{S^{n}(A) \mid A \in \mathcal{A} \text { and } n \in \mathbb{Z}\right\}^{\perp} \text { and } \tilde{\mathcal{A}}={ }^{\perp}\left\{S^{n}(B) \mid B \in \mathcal{B} \text { and } n \in \mathbb{Z}\right\}
$$

In particular we have cotorsion pairs $\left({ }^{\perp} \tilde{\mathcal{B}}, \tilde{\mathcal{B}}\right)$ and $\left(\tilde{\mathcal{A}}, \tilde{\mathcal{A}}^{\perp}\right)$ in $\mathbf{C}(\mathcal{G})$.

Proof. Denote $\mathcal{X}=\left\{S^{n}(A) \mid A \in \mathcal{A} \text { and } n \in \mathbb{Z}\right\}^{\perp}$. Since $\mathcal{A}$ is generating, the class $\mathcal{X}$ consists of acyclic complexes. Indeed, component wise split short exact sequences $0 \rightarrow X \rightarrow E \rightarrow S^{n}(A) \rightarrow 0$ must split for every $X \in \mathcal{X}$, so that every cochain complex homomorphism $S^{n+1}(A) \rightarrow X$ must be null-homotopic, and consequently $X^{n} \rightarrow Z^{n+1}(X)$ must be an epimorphism for every $n \in \mathbb{Z}$. Then $\mathcal{X} \subseteq \tilde{\mathcal{B}}$ by Lemma 7.12 The inclusion $\mathcal{X} \supseteq \tilde{\mathcal{B}}$ is easy and the other case is dual.

The important feature is completeness of $\left({ }^{\perp} \tilde{\mathcal{B}}, \tilde{\mathcal{B}}\right)$ and $\left(\tilde{\mathcal{A}}, \tilde{\mathcal{A}}^{\perp}\right)$, which we can prove for Grothendieck categories.

Proposition 7.14. Let $\mathcal{G}$ be a Grothendieck category and $(\mathcal{A}, \mathcal{B})$ be a complete hereditary cotorsion pair in $\mathcal{G}$. Then $\left({ }^{\perp} \tilde{\mathcal{B}}, \tilde{\mathcal{B}}\right)$ and $\left(\tilde{\mathcal{\mathcal { A }}}, \tilde{\mathcal{A}}^{\perp}\right)$ are complete (and hereditary) cotorsion pairs in $\mathbf{C}(\mathcal{G})$.

Proof. The pair $\left(\tilde{\mathcal{A}}, \tilde{\mathcal{A}}^{\perp}\right)$ is complete by Lemmas 7.9 and 7.10 For $\left({ }^{\perp} \tilde{\mathcal{B}}, \tilde{\mathcal{B}}\right)$, let $G \in \mathcal{A}$ be a generator for $\mathcal{G}$ and consider the cotorsion pair $\left(\mathcal{P}, \mathcal{P}^{\perp}\right)$ generated from the left hand side by the set $\left\{S^{n}(G) \mid n \in \mathbb{Z}\right\}$. Then $\mathcal{P} \subseteq{ }^{\perp} \tilde{\mathcal{B}}$ by Lemma 7.13 and also $\mathcal{P}^{\perp} \subseteq \mathbf{C}_{\text {ac }}(\mathcal{G})$. Now we can use the dual version of the proof for Lemma 7.10, just taking the corresponding approximations with respect to $\left(\mathcal{P}, \mathcal{P}^{\perp}\right)$ instead of the ones for $\left(\tilde{\mathcal{G}}, \tilde{\mathcal{G}}^{\perp}\right)$. 
Remark 7.15. Gillespie [27, 28, 29] denotes ${ }^{\perp} \tilde{\mathcal{B}}$ by $d g \tilde{\mathcal{A}}$ and $\tilde{\mathcal{A}}^{\perp}$ by $d g \tilde{\mathcal{B}}$. If $\mathcal{A}$ is deconstructible in $\mathcal{G}$, i.e. $\mathcal{A}=$ Filt $\mathcal{S}$ for a set of objects $\mathcal{S}$, then both $\tilde{\mathcal{A}}$ and $d g \tilde{\mathcal{A}}$ are deconstructible by [67, Theorem 4.2]. This implies that $(d g \tilde{\mathcal{A}}, \tilde{\mathcal{B}})$ and $(\tilde{\mathcal{A}}, d g \tilde{\mathcal{B}})$ are functorially complete and the model structure from Theorem 7.16 is cofibrantly generated (see Remark 6.10). The objects of $d g \tilde{\mathcal{A}}$ are then characterized as retracts of objects in Filt $\left\{S^{n}(X) \mid X \in \mathcal{S}\right.$ and $\left.n \in \mathbb{Z}\right\}$ (see also [67, Proposition 4.5]).

Now we can state and prove the result on the existence of models for $\mathbf{D}(\mathcal{G})$.

Theorem 7.16. Let $\mathcal{G}$ be a Grothendieck category and $(\mathcal{A}, \mathcal{B})$ a complete hereditary cotorsion pair in $\mathcal{G}$. Then there is a hereditary model structure on $\mathbf{C}(\mathcal{G})$ such that

(1) The class of cofibrant objects equals ${ }^{\perp} \tilde{\mathcal{B}}$ (using Notation 7.6).

(2) The trivial objects are precisely the acyclic complexes, $\mathcal{W}=\mathbf{C}_{\mathrm{ac}}(\mathcal{G})$.

(3) The class of fibrant objects equals is $\tilde{\mathcal{A}}^{\perp}$.

In particular we have $\mathrm{Ho} \mathbf{C}(\mathcal{G})=\mathbf{D}(\mathcal{G})$, the unbounded derived category of $\mathcal{G}$.

Proof. We lift $(\mathcal{A}, \mathcal{B})$ to a complete cotorsion pair $(\tilde{\mathcal{A}}, \tilde{\mathcal{B}})$ in $\mathbf{C}_{\mathrm{ac}}(\mathcal{G})$ and use Proposition 7.14 to prove that $\left({ }^{\perp} \tilde{\mathcal{B}}, \tilde{\mathcal{B}}\right)$ and $\left(\tilde{\mathcal{A}}, \tilde{\mathcal{A}}^{\perp}\right)$ are complete cotorsion pairs in $\mathbf{C}(\mathcal{G})$. The hereditary model structure determined by $(\mathcal{C}, \mathcal{W}, \mathcal{F})=\left({ }^{\perp} \tilde{\mathcal{B}}, \mathbf{C}_{\mathrm{ac}}(\mathcal{G}), \tilde{\mathcal{A}}^{\perp}\right)$ is obtained from Proposition 7.1. The weak equivalences are usual quasi-isomorphism by the same argument as for Theorem 7.11, so that $\operatorname{Ho} \mathbf{C}(\mathcal{G})=\mathbf{D}(\mathcal{G})$.

7.4. Neeman's pure derived category of flat modules. The last example that we discuss here is due to Neeman [57. Although model categories are not mentioned at all in [57, there is a direct interpretation of the main results in our language. Basically, Neeman considers two model structures for the derived category of flat modules over a ring - the injective one described in Section 7.2 and the projective one described in the following theorem.

The significance of $\mathbf{D}$ (Flat- $R$ ) is that for a left coherent ring $R$ it is compactly generated and the category of compact objects is triangle equivalent to $\mathbf{D}^{\mathrm{b}}\left(\bmod -R^{\mathrm{op}}\right)^{\mathrm{op}}$, the opposite category of the bounded derived category of the category mod- $R^{\mathrm{op}}$ of finitely presented left modules. If $R$ is commutative noetherian, there are interesting connections to the classical Grothendieck duality [36].

Theorem 7.17. Let $R$ be a ring and Flat- $R$ be the category of all flat right $R$ modules with the induced exact structure. Then there is a cofibrantly generated hereditary model structure on $\mathbf{C}($ Flat- $R$ ) such that

(1) The class of cofibrant objects is precisely $\mathbf{C}(\operatorname{Proj}-R)$, the complexes with projective components.

(2) The trivial objects are precisely the acyclic complexes, $\mathcal{W}=\mathbf{C}_{\mathrm{ac}}($ Flat- $R)$.

(3) Every object of $\mathbf{C}($ Flat-R) is fibrant.

In particular we have Ho $\mathbf{C}($ Flat $-R)=\mathbf{D}($ Flat $-R)$. 
Proof. In view of [65, §3] Neeman proves in [57, Theorems 8.6 and 5.9] that $(\mathbf{C}(\operatorname{Proj}-R), \mathcal{W})$ is a complete cotorsion pair in $\mathbf{C}($ Flat- $R)$ generated by a set $\mathcal{S}$ as in Theorem 5.16 and that $\mathbf{C}(\operatorname{Proj}-R) \cap \mathcal{W}=\mathbf{C}_{\text {ac }}(\operatorname{Proj}-R)$. One also easily checks that $\mathbf{C}_{\mathrm{ac}}(\operatorname{Proj}-R)$ are precisely the projective objects in $\mathbf{C}$ (Flat- $R$ ). Thus we see that if $\mathcal{A}=\operatorname{Proj}-R$ and $\mathcal{B}=$ Flat- $R$, then $(\mathcal{A}, \mathcal{B})$ is a complete hereditary cotorsion pair in Flat- $R$ and both $\left({ }^{\perp} \tilde{\mathcal{B}}, \tilde{\mathcal{B}}\right)$ and $\left(\tilde{\mathcal{A}}, \tilde{\mathcal{A}}^{\perp}\right)$ are complete in $\mathbf{C}($ Flat- $R)$. It remains to apply Proposition 7.1 and Remark 6.10,

Another main result of [57] comes as an easy consequence.

Corollary 7.18. Let $R$ be a ring. Then $\mathbf{D}$ (Flat- $R$ ) is triangle equivalent to $\mathbf{K}(\operatorname{Proj}-R)$, the homotopy category of complexes of projective modules.

Proof. By restriction, the model structure on $\mathbf{C}($ Flat- $R$ ) induces a hereditary model structure on $\mathbf{C}(\operatorname{Proj}-R)$ given by

$$
(\mathcal{C}, \mathcal{W}, \mathcal{F})=\left(\mathbf{C}(\operatorname{Proj}-R), \mathbf{C}_{\text {ac }}(\operatorname{Proj}-R), \mathbf{C}(\operatorname{Proj}-R)\right) .
$$

There is an obvious functor $\mathrm{Ho} \mathbf{C}(\operatorname{Proj}-R) \rightarrow$ Ho $\mathbf{C}$ (Flat- $R$ ) which is a triangle equivalence by [31, Corollary 5.4] and Theorem 6.21. Moreover, Ho $\mathbf{C}(\operatorname{Proj}-R)$ is none other than $\mathbf{K}($ Proj- $R$ ) by Lemma 6.16(3).

\section{Monoidal model categories from flat sheaves and vector bundles}

In the final section we will consider exact model structures which are compatible with a symmetric monoidal product on the underlying category. As an illustration we will show, under appropriate assumptions, constructions of model structures compatible with the tensor product for the derived category of the category of quasi-coherent sheaves over a scheme.

8.1. Tensor product for quasi-coherent modules. Let us briefly recall what a closed symmetric monoidal category is, and refer to [51, Chapter VII] for details. A category $\mathcal{C}$ is called symmetric monoidal if it is equipped with a functor $\otimes: \mathcal{C} \times$ $\mathcal{C} \rightarrow \mathcal{C}$, often called tensor product, and an object $\mathbb{1} \in \mathcal{C}$ such that $\otimes$ is associative, commutative and $\mathbb{1}$ is a unit with respect to $\otimes$. More precisely, there are natural isomorphisms $X \otimes(Y \otimes Z) \cong(X \otimes Y) \otimes Z, X \otimes Y \cong Y \otimes X$ and $\mathbb{1} \otimes X \cong X$ satisfying certain coherence conditions. A symmetric monoidal category is called closed if the functor $-\otimes Y$ has a right adjoint for each $Y \in \mathcal{C}$. If we for each $Y$ fix such an adjoint and denote it by $\mathcal{H o m}(Y,-)$, it is a standard fact that $\mathcal{H o m}$ is a functor $\mathcal{C}^{\mathrm{op}} \times \mathcal{C} \rightarrow \mathcal{C}$ and we obtain an isomorphism

$$
\mathcal{C}(X \otimes Y, Z) \stackrel{\cong}{\longrightarrow} \mathcal{C}(X, \mathcal{H o m}(Y, Z)),
$$

which is natural in all three variables $X, Y, Z$.

If $R: I \rightarrow$ CommRings is a flat representation of a poset in the category of commutative rings (Definitions 2.3 and 2.9), then $\mathrm{Q} \operatorname{coh}(R)$ is a closed symmetric 
monoidal category. Indeed, using the notation from Definition 2.6 we define $L \otimes M$ by $(L \otimes M)(i)=L(i) \otimes_{R(i)} M(i)$ and $(L \otimes M)_{j}^{i}=L_{j}^{i} \otimes M_{j}^{i}$ for each $i \leq j$ from $I$. The tensor unit $\mathbb{1}$ is clearly $R$ itself viewed as an object of $\mathrm{Q} \operatorname{coh}(R)$.

It is not difficult to see that $\mathrm{Q} \operatorname{coh}(R)$ is closed, see [56, Proposition 6.15]. Indeed, for $M, N \in \mathrm{Q} \operatorname{coh}(R)$ we can define an $R$-module $\underline{\mathcal{H} o m}(M, N) \in$ Mod- $R$ by putting $\underline{\mathcal{H} o m}(M, N)(i)=\operatorname{Hom}_{R(i)}(M(i), N(i))$ for each $i \in I$, and use the quasicoherence of $M$ and $N$ to define the maps inside $\underline{\mathcal{H} o m}(M, N)$ as $\underline{\mathcal{H} o m}(M, N)_{j}^{i}: f \mapsto$ $f \otimes_{R(i)} R(j)$ for $i \leq j$. Although we obtain the adjunction

$$
\operatorname{Hom}_{R}(L \otimes M, N) \stackrel{\cong}{\longrightarrow} \operatorname{Hom}_{R}(L, \underline{\mathcal{H o m}}(M, N))
$$

the $R$-module $\underline{\mathcal{H} o m}(M, N)$ may not be quasi-coherent unless all the $M(i)$ are finitely presented over $R(i)$. We can fix the problem by defining $\mathcal{H o m}(M, N)=$ $Q(\underline{\mathcal{H} o m}(M, N))$, where $Q$ is the coherator from Remark 2.12

There is a standard way to extend this to a closed symmetric monoidal structure on $\mathbf{C}(\mathrm{Q} \operatorname{coh}(R))$. Given $X, Y \in \mathbf{C}(\mathrm{Q} \operatorname{coh}(R))$, we define components of the complex $X \otimes Y$ by

$$
(X \otimes Y)^{n}=\coprod_{i+j=n} X^{i} \otimes Y^{j} \quad \text { for each } \mathrm{n} \in \mathbb{Z}
$$

and the differentials by $\left.\partial\right|_{X^{i} \otimes Y^{j}}=\partial_{X}^{i} \otimes Y^{j}+(-1)^{i} X^{i} \otimes \partial_{Y}^{j}$ for all $i, j \in \mathbb{Z}$. The unit $\mathbb{1}$ is the complex $S^{0}(R)$ (Notation 7.4). Similarly, for $Y, Z \in \mathbf{C}(\mathrm{Q} \operatorname{coh}(R))$, the complex $\mathcal{H o m}(Y, Z)$ is defined by

$$
\mathcal{H o m}(Y, Z)^{n}=\prod_{j-i=n} \mathcal{H o m}\left(Y^{i}, Z^{j}\right) \quad \text { for each } \mathrm{n} \in \mathbb{Z}
$$

where the product is taken in $\operatorname{Qcoh}(R)$ (see Remark 2.12), and by $\left.\partial\right|_{\mathcal{H o m}\left(Y^{i}, Z^{j}\right)}=$ $\mathcal{H o m}\left(Y^{i}, \partial_{Z}^{j}\right)-(-1)^{j-i} \mathcal{H o m}\left(\partial_{Y}^{i}, Z^{j}\right)$ for all $i, j \in \mathbb{Z}$.

This closed symmetric monoidal category $(\mathbf{C}(\mathrm{Q} \operatorname{coh}(R)), \otimes, \mathbb{1}, \mathcal{H}$ om $)$ is the one to keep in mind in the discussion to come.

8.2. Derived functors. In order to give a good sense to what a compatibility of a monoidal structure with a model structure means, we need to give a brief account on derived functors. We refer to [38, $\S \S 8.4$ and 8.5$]$ for more details. After all, what we need to do is to construct the derived functors of $\otimes: \mathcal{C} \times \mathcal{C} \rightarrow \mathcal{C}$ and Hom: $\mathcal{C}^{\text {op }} \times \mathcal{C} \rightarrow \mathcal{C}$.

Let $\mathcal{C}, \mathcal{D}$ be model categories and $F: \mathcal{C} \rightarrow \mathcal{D}$ be a functor. We would like to define a functor Ho $F$ making the diagram

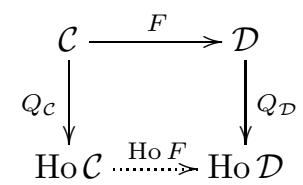

commutative, but we usually cannot as $F$ typically does not send weak equivalences to weak equivalences. What we usually can do, however, is to find the "best approximation" from the left or from the right. 
Definition 8.1. 38, 8.4.1 and 8.4.7] Let $F: \mathcal{C} \rightarrow \mathcal{D}$ be a functor between model categories $\mathcal{C}$ and $\mathcal{D}$. A total left derived functor of $F$ is a functor $\mathbf{L} F: \mathrm{HoC}_{\rightarrow} \rightarrow$ Ho $\mathcal{D}$ together with a natural transformation $\varepsilon: \mathbf{L} F \circ Q_{\mathcal{C}} \rightarrow Q_{D} \circ F$ satisfying the following universal property. If $G: \operatorname{Ho} \mathcal{C} \rightarrow \mathrm{Ho} \mathcal{D}$ is another functor with a natural transformation $\zeta: G \circ Q_{\mathcal{C}} \rightarrow Q_{D} \circ F$, then there is a unique natural transformation $\vartheta: G \rightarrow \mathbf{L} F$ such that $\zeta=\varepsilon\left(\vartheta \circ Q_{\mathcal{C}}\right)$.

A total right derived functor of $F$ is a functor $\mathbf{R} F:$ Ho $\mathcal{C} \rightarrow$ Ho $\mathcal{D}$ together with a natural transformation $\eta: Q_{D} \circ F \rightarrow \mathbf{R} F \circ Q_{\mathcal{C}}$ which satisfies the obvious dual universal property.

That is, we are often in the situation of one of the following universal squares:
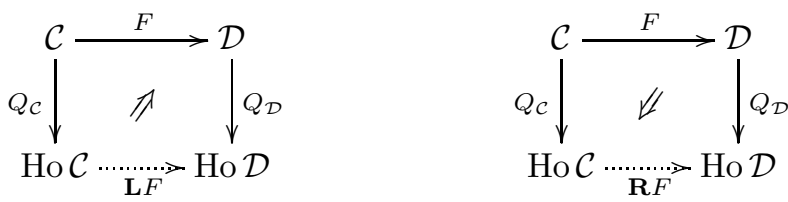

The key point is that if $\mathbf{L} F$ (or $\mathbf{R} F$ ) exists, it is defined uniquely up to a unique isomorphism, and does not depend on the actual model structures on $\mathcal{C}$ and $\mathcal{D}$, but only on the classes of weak equivalences in $\mathcal{C}$ and $\mathcal{D}$.

We shall list standard criteria for existence of total derived functors.

Proposition 8.2. Let $F: \mathcal{C} \rightarrow \mathcal{D}$ be a functor between model categories $\mathcal{C}$ and $\mathcal{D}$.

(1) If $F$ takes trivial cofibrations between cofibrant objects in $\mathcal{C}$ to weak equivalences in $\mathcal{D}$ (see also Lemma 6.14 for exact model categories), then the total left derived functor $\mathbf{L} F: \operatorname{Ho} \mathcal{C} \rightarrow$ Ho $\mathcal{D}$ exists.

(2) If $F$ takes trivial fibrations between fibrant objects in $\mathcal{C}$ to weak equivalences in $\mathcal{D}$, then the total right derived functor $\mathbf{R} F: \operatorname{Ho} \mathcal{C} \rightarrow \operatorname{Ho} \mathcal{D}$ exists.

Proof. This is just [38, Proposition 8.4.8], but as we dropped the assumption of functoriality of the factorizations in Definition 6.1 (MS2) while [38] uses the functoriality, we indicate the necessary modification in the proof of [38, Proposition 8.4.4]. Suppose we are in the situation of (1). Then we construct $\mathbf{L} F$ as follows. Given $X \in \mathcal{C}$, we fix a cofibrant replacement $f_{X}: C X \rightarrow X$ of $X$ (see Definition 6.3) and put $\mathbf{L} F(X)=Q_{D} F(C X)$. Given a morphism $u: X \rightarrow Y$ in $\mathcal{C}$, we choose $f_{u}$ to make the square

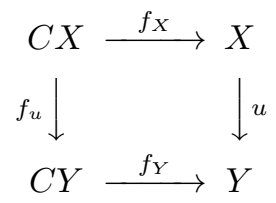

commute and put $\mathbf{L} F(u)=Q_{D} F\left(f_{u}\right)$. This is well defined since if $f_{u}^{\prime}: C X \rightarrow C Y$ is another map which fits into the square, then $f_{u}$ and $f_{u}^{\prime}$ are left homotopic (see also Lemma 6.16 for exact model categories), hence $Q_{D} F\left(f_{u}\right)=Q_{D} F\left(f_{u}^{\prime}\right)$. The rest of the proof is as in [38] and case (2) is dual. 
Example 8.3. As this formalism is still somewhat unusual in homological algebra, we shall illustrate it on the probably most usual situation with derived categories. Let $R, S$ be rings and $F: \operatorname{Mod}-R \rightarrow \operatorname{Mod}-S$ be an additive functor. Then we have an obvious functor $F: \mathbf{C}(\operatorname{Mod}-R) \rightarrow \mathbf{C}(\operatorname{Mod}-S)$, which we also denote by $F$. Now we equip $\mathbf{C}(\operatorname{Mod}-R)$ and $\mathbf{C}(\operatorname{Mod}-S)$ with the two extremal hereditary model structures coming from Theorem 7.16. We use the cotorsion pair (Proj- $R$, Mod- $R$ ) for $\mathbf{C}(\operatorname{Mod}-R)$ and the cotorsion pair $(\operatorname{Mod}-S, \operatorname{Inj}-S)$ for $\mathbf{C}(\operatorname{Mod}-S)$. It is an easy observation that $F$ satisfies Proposition $8.2(1)$. Thus $\mathbf{L} F: \mathbf{D}(\operatorname{Mod}-R) \rightarrow \mathbf{D}($ Mod- $S)$ exists.

In connection with closed symmetric monoidal categories, we also need to know how derived functors of adjoint pairs behave. Typically if $(F, U)$ is an adjoint pair of functors and if $\mathbf{L} F$ and $\mathbf{R} G$ exist, they also form an adjoint pair. A very general setting where this holds has been described by Maltsiniotis in [53. For simplicity, we only state the result for classical Quillen pairs of functors.

Proposition 8.4. Let $\mathcal{C}, \mathcal{D}$ be model categories and $(F, U)$, where $F: \mathcal{C} \rightarrow \mathcal{D}$ and $U: \mathcal{D} \rightarrow \mathcal{C}$, be an adjoint pair of functors. Then the following are equivalent:

(1) The left adjoint $F$ preserves both cofibrations and trivial cofibrations.

(2) The right adjoint $U$ preserves both fibrations and trivial fibrations.

In such a case, both $\mathbf{L} F$ and $\mathbf{R} U$ exist and $(\mathbf{L} F, \mathbf{R} U)$ is an adjoint pair.

Proof. This is proved in [38, Proposition 8.5.3 and Theorem 8.5.18].

Definition 8.5. Adjoint pairs $(F, U)$ as in Proposition 8.4 are called Quillen pairs.

8.3. Monoidal model categories. Now we are left with a relatively easy task: given a closed symmetric monoidal category, determine which condition a model structure on $\mathcal{C}$ must satisfy so that we can use it to construct the derived functors of $\otimes: \mathcal{C} \times \mathcal{C} \rightarrow \mathcal{C}$ and $\mathcal{H}$ om $: \mathcal{C}^{\text {op }} \times \mathcal{C} \rightarrow \mathcal{C}$. Note that if $\mathcal{C}$ and $\mathcal{D}$ carry model structures, then $\mathcal{C} \times \mathcal{D}$ admits a so-called product model structure, where a morphism is a cofibration, a weak equivalence, or a fibration, respectively, if both the components in $\mathcal{C}$ and $\mathcal{D}$ are such (cf. [41, Example 1.1.6]). Similarly, if $\mathcal{C}$ is a model category, $\mathcal{C}^{\text {op }}$ is naturally a model category too, by swapping the roles of fibrations and cofibrations (see [41, Remark 1.1.7]).

Remark 8.6. An important point is that for computing the derived functor of $\otimes: \mathcal{C} \times \mathcal{C} \rightarrow \mathcal{C}$, we need not equip all three copies of $\mathcal{C}$ with the same model structure. Indeed, if $R$ is a commutative ring and we are to compute $X \otimes_{R}^{\mathbf{L}} Y$ in $\mathcal{C}=\mathbf{D}(\operatorname{Mod}-R)$, it is enough to find a suitable flat resolution for $X$ only. By doing so, we actually consider the first copy of $\mathcal{C}$ with the model structure given by Theorem $\left[7.16\right.$ for the cotorsion pair (Flat- $R$, Flat- $R^{\perp}$ ) (see also Section [8.4), while the other two copies of $\mathcal{C}$ are considered with the injective model structure given by the trivial cotorsion pair $(\operatorname{Mod}-R, \operatorname{Inj}-R)$. 
For theoretical reasons, however, it is desirable to be able to equip $\mathcal{C}$ with a single model structure which can be used for computing both the total left derived functor $\otimes^{\mathbf{L}}$ of $\otimes$ and the total right derived functor $\mathbf{R} \mathcal{H o m}$ of $\mathcal{H o m}$. A condition which the model structure on $\mathcal{C}$ should satisfy is stated in the following definition.

Definition 8.7. Let $\mathcal{C}, \mathcal{D}, \mathcal{E}$ be model categories. A functor $\otimes: \mathcal{C} \times \mathcal{D} \rightarrow \mathcal{E}$ is called a Quillen bifunctor if the following condition is satisfied. If $f: U \rightarrow V$ is a cofibration in $\mathcal{C}$ and $g: X \rightarrow Y$ is a cofibration in $\mathcal{D}$, we require that the map $f \otimes g$ defined as the dotted arrow in the following pushout diagram (which is assumed to exist)

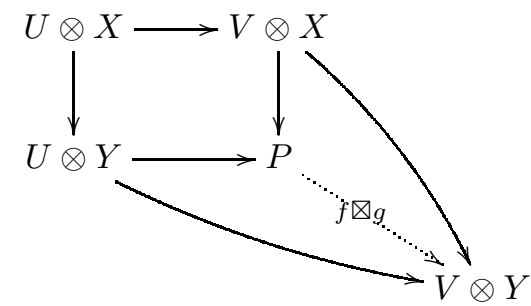

be a cofibration in $\mathcal{E}$. Moreover, if one of $f$ and $g$ is trivial, we require $f \otimes g$ to be trivial.

The following lemma is standard.

Lemma 8.8. [41, 4.2.2] Let $\mathcal{C}, \mathcal{D}, \mathcal{E}$ be model categories and let $\otimes: \mathcal{C} \times \mathcal{D} \rightarrow \mathcal{E}$ be a Quillen bifunctor. If $\mathcal{H}$ om: $\mathcal{D}^{o p} \times \mathcal{E} \rightarrow \mathcal{C}$ is a functor such that we have a natural isomorphism

$$
\mathcal{E}(X \otimes Y, Z) \stackrel{\cong}{\longrightarrow}(X, \mathcal{H o m}(Y, Z)),
$$

in all three variables, then $\mathcal{H}$ om is a Quillen bifunctor when we view it as a functor $\mathcal{D} \times \mathcal{E}^{o p} \rightarrow \mathcal{C}^{o p}$ of the opposite categories.

Now we can present a slightly simplified version of [41, Definition 4.2.6] along with the expected theorem.

Definition 8.9. A monoidal model category is a model category $\mathcal{C}$ (Definition 6.1) which carries a closed symmetric monoidal structure $(\mathcal{C}, \otimes, \mathbb{1}, \mathcal{H o m})$ such that

(MS3) The tensor unit $\mathbb{1}$ is cofibrant.

(MS4) The product $\otimes: \mathcal{C} \times \mathcal{C} \rightarrow \mathcal{C}$ is a Quillen bifunctor.

An exact monoidal model category and hereditary monoidal model category are the corresponding variants where $\mathcal{C}$ is an exact model category (Definition 6.8) or hereditary model category (Definition 6.19), respectively.

Theorem 8.10. Let $\mathcal{C}$ be a monoidal model category. Then the total derived functors

$$
\otimes^{\mathbf{L}}: \mathrm{Ho} \mathcal{C} \times \mathrm{Ho} \mathcal{C} \longrightarrow \mathrm{Ho} \mathcal{C} \text { and } \mathbf{R H o m}: \mathrm{Ho}^{o p} \times \mathrm{Ho} \mathcal{C} \longrightarrow \mathrm{Ho} \mathcal{C}
$$

exist and $\left(\mathrm{Ho} \mathcal{C}, \otimes^{\mathbf{L}}, \mathbb{1}, \mathbf{R} \mathcal{H}\right.$ om $)$ is a closed symmetric monoidal category. 
Proof. This is just a special case of [41, Theorem 4.3.2], but at this point we have all the tools to understand the proof. Since $\otimes$ is a Quillen bifunctor, $f \otimes g: U \otimes X \rightarrow$ $V \otimes Y$ is a cofibration whenever $f: U \rightarrow V$ and $g: X \rightarrow Y$ are cofibrations, and $f \otimes g$ is a trivial cofibration if one of $f, g$ is such. Similarly we deduce from Lemma 8.8 that $\mathcal{H o m}(g, p)$ is a fibration whenever $g$ is a cofibration and $p$ is a fibration, and it is a trivial fibration if one of $g, p$ is trivial. In particular, the total left derived functor $\otimes^{\mathbf{L}}$ of $\otimes: \mathcal{C} \times \mathcal{C} \rightarrow \mathcal{C}$ and the total right derived functor $\mathbf{R}$ Hom of $\mathcal{H o m}: \mathcal{C}^{\text {op }} \times \mathcal{C} \rightarrow \mathcal{C}$ exist by Proposition 8.2

It also follows that if $X$ is cofibrant, then $(-\otimes X, \mathcal{H o m}(X,-))$ is a Quillen pair. Thus $\left(-\otimes^{\mathbf{L}} X, \mathbf{R H o m}(X,-)\right)$ is a pair of adjoint functors by Proposition 8.4 .

Using the construction of $\otimes^{\mathbf{L}}$ in the proof of Proposition 8.2 , one also sees that $-\otimes^{\mathbf{L}}\left(-\otimes^{\mathbf{L}}-\right)$ coincides with the total left derived functor of $-\otimes(-\otimes-)$, and $\left(-\otimes^{\mathbf{L}}-\right) \otimes^{\mathbf{L}}-$ is the left derived functor of $(-\otimes-) \otimes-$. Hence the associativity transformation for $\otimes$ lifts to associativity for $\otimes^{\mathbf{L}}$, using the universal property of the derived functors. The commutativity transformation for $\otimes^{\mathbf{L}}$ and the isomorphism $\mathbb{1} \otimes^{\mathbf{L}}-\cong 1_{\text {Ho } \mathcal{C}}$ are obtained similarly. Finally, the transformations for $\otimes^{\mathbf{L}}$ satisfy the coherence conditions since they were constructed from the transformations for $\otimes$ using the universal property.

Naturally we are interested in what conditions a triple of classes $(\mathcal{C}, \mathcal{W}, \mathcal{F})$ as in Theorem 6.9 must satisfy in order to define an exact or hereditary monoidal model structure. Now we can give a relatively easy answer, which was first obtained in [42, Theorem 7.2].

Theorem 8.11. [42, 7.2] Let $\mathcal{E}$ be an exact model category given in the sense of Theorem 6.9 by the triple $(\mathcal{C}, \mathcal{W}, \mathcal{F})$ of cofibrant, trivial, and fibrant objects, respectively. Suppose further that $(\mathcal{E}, \otimes, \mathbb{1}, \mathcal{H o m})$ is also a closed symmetric monoidal category. Then $\mathcal{E}$ with all the structure forms an exact monoidal model category provided that

(1) The tensor unit $\mathbb{1}$ is cofibrant.

(2) If $X, Y \in \mathcal{C}$, then $X \otimes Y \in \mathcal{C}$. If, moreover, $X$ or $Y$ is in $\mathcal{C} \cap \mathcal{W}$, then $X \otimes Y \in \mathcal{C} \cap \mathcal{W}$.

(3) If $f: X \rightarrow Y$ is a cofibration and $U \in \mathcal{E}$ is arbitrary, then $U \otimes f: U \otimes X \rightarrow$ $U \otimes Y$ is an inflation in $\mathcal{E}$.

Remark 8.12. Note a subtle point here: In principle the definition of a monoidal model category requires some extra pushouts to exist so that the maps $f \otimes g$ from Definition 8.7 are defined for any pair of cofibrations $f, g$. Condition (3) from the latter theorem ensures, however, that no extra pushouts except for those guaranteed by the axioms of an exact category are required.

Proof of Theorem 8.11. We only need to show that (2) and (3) imply that $\otimes$ is a Quillen bifunctor (see Definition 8.7). To this end, let $f: U \rightarrow V$ and $g: X \rightarrow Y$ be 
cofibrations with cokernels $C$ and $D$, respectively. Using (3) and 10, Proposition 3.1 , we construct the following commutative diagram with conflations in rows:

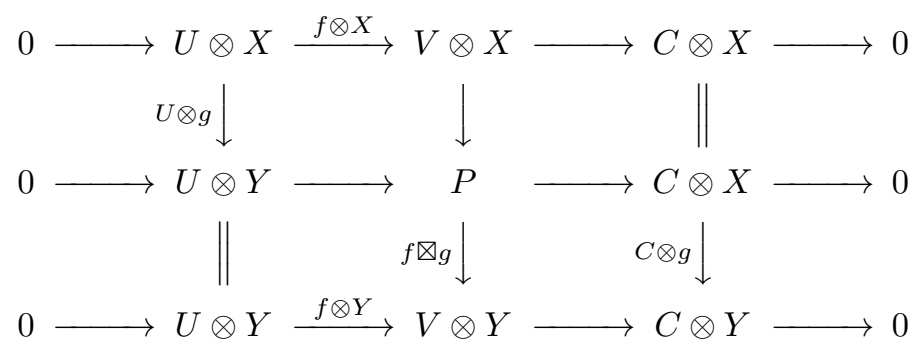

Thanks to [10, Proposition 2.12], we have a conflation

$$
0 \longrightarrow P \stackrel{f \otimes g}{\longrightarrow} V \otimes Y \longrightarrow C \otimes D \longrightarrow 0 .
$$

Now the conclusion follows from assumption (2).

8.4. Complexes of flat quasi-coherent modules. The last task is to instantiate Theorem 8.11 for the closed symmetric monoidal structure on $\mathbf{C}(\mathrm{Q} \operatorname{coh}(R))$ discussed in 88.1 . The hardest part is to fulfil Theorem $8.11(3)$, as this typically does not hold for the injective model structure on $\mathbf{C}(\mathrm{Q} \operatorname{coh}(R))$ from Theorem 7.11. Eventually we will need to impose more assumptions on the flat representation $R: I \rightarrow$ CommRings, but let us first discuss the aspects which work in full generality.

Recall from $\$ 2.2$ that a left module $M$ over a ring $T$ is called flat if the functor $-\otimes_{T} M:$ Mod- $T \rightarrow \mathrm{Ab}$ is exact. By Lazard's theorem [32, Corollary 1.2.16], $M$ is flat if and only if it is a direct limit of finitely generated projective modules.

Analogously we call $M \in \mathrm{Q} \operatorname{coh}(R)$ flat if the functor $-\otimes M: \mathrm{Q} \operatorname{coh}(R) \rightarrow$ $\mathrm{Q} \operatorname{coh}(R)$ is exact. This notion of flatness corresponds to the classical one for quasi-coherent sheaves if $R$ comes from a scheme in the sense of 2.3 It is easy to check that $M$ is flat if and only if $M(i)$ is a flat module over $R(i)$ for each $i \in I$. We shall denote the class of all flat quasi-coherent modules over $R$ by Flat- $R$. Our aim is to use Corollary 5.17 to show that there is often a complete hereditary cotorsion pair (Flat- $R$, Flat- $R^{\perp}$ ). We denote the class Flat- $R^{\perp}$ by Cot- $R$ and, as customary, we call the quasi-coherent modules inside it cotorsion.

First of all, the class of left flat modules over a $\operatorname{ring} T$ is always deconstructible by the proof of [32, Theorem 3.2.9], and it is a left hand side class of a complete hereditary cotorsion pair by [32, Theorem 4.1.1]. Regarding the deconstructibility of Flat- $R$ for $R: I \rightarrow$ CommRings, we can use the following technical result, whose proof involves the Hill Lemma (see Proposition 3.14).

Proposition 8.13. Let $R: I \rightarrow$ Rings be a flat representation of $I$ in the category of rings, and suppose that we are given for each $i \in I$ a deconstructible class $\mathcal{C}_{i} \subseteq \operatorname{Mod}-R(i)$. Then the class $\mathcal{C} \subseteq \mathrm{Q} \operatorname{coh}(R)$ defined as

$$
\mathcal{C}=\left\{M \in \operatorname{Qcoh}(R) \mid M(i) \in \mathcal{C}_{i} \text { for each } i \in I\right\}
$$

is deconstructible in $\mathrm{Q} \operatorname{coh}(R)$. 
Proof. The same proof as for [23, Theorem 3.13 and Corollary 3.14] applies.

Corollary 8.14. If $R: I \rightarrow$ CommRings is a flat representation, then the class Flat- $R$ is deconstructible in $\mathrm{Qcoh}(R)$ and it is closed under retracts and kernels of epimorphisms.

Thus, in view of Corollary 5.17 and Lemma 6.17, the following is sufficient and necessary in order to obtain a complete hereditary cotorsion pair.

Corollary 8.15. If $R: I \rightarrow$ CommRings is a flat representation, then there exists $a$ (functorially) complete hereditary cotorsion pair (Flat-R, Cot- $R$ ) in $\mathrm{Qcoh}(R)$ if and only if $\mathrm{Qcoh}(R)$ admits a flat generator.

Constructing a flat generator is indeed the most tricky part. It exists in $\mathrm{Qcoh}(X)$ for any quasi-compact and separated scheme $X$, as has been implicitly proved in [3, Proposition 1.1] and discussed explicitly and in detail by Murfet in $56, \S 3.2]$.

To formulate this in our formalism from $\$ 2.2$, recall that a poset $(I, \leq)$ is an upper semilattice if every pair $x, y \in I$ has a least upper bound $x \vee y$. We will call a representation $R: I \rightarrow$ CommRings of an upper semilattice $I$ continuous if it preserves pushouts. In other words, if $x, y, z \in I$ such that $x \leq y$ and $x \leq z$, then the ring homomorphism

$$
R_{y \vee z}^{y} \otimes_{R(x)} R_{y \vee z}^{z}: R(y) \otimes_{R(x)} R(z) \rightarrow R(y \vee z)
$$

is required to be bijective. If $X$ is a quasi-compact separated scheme, then the results from $₫ 2.3$ can be used to construct an equivalence $Q \operatorname{coh}(R) \stackrel{\cong}{\longrightarrow} \mathrm{Qcoh}(X)$ for a continuous flat representation $R$ of a finite upper semilattice $I$. This explains the motivation for the following statement which (at least formally) slightly generalizes the above mentioned result from [3, 56].

Proposition 8.16. Let $I$ be a finite upper semilattice and $R: I \rightarrow$ CommRings be a continuous flat representation. Then $\mathrm{Q} \operatorname{coh}(R)$ admits a flat generator. In particular, there is a functorially complete hereditary cotorsion pair (Flat-R, Cot-R) in $\mathrm{Q} \operatorname{coh}(R)$.

Proof. We shall closely follow the structure of the proof from [56. Let us fix a finite number of elements $x_{0}, x_{1}, \ldots, x_{n} \in I$ such that every $x \in I$ is of the form $x=x_{i_{0}} \vee \cdots \vee x_{i_{p}}$ for some $0 \leq i_{0}<\cdots<i_{p} \leq n$. Since $R$ is a continuous representation, the functor

$$
F_{x}^{*}: \operatorname{Qcoh}(R) \longrightarrow \operatorname{Mod}-R(x), \quad M \longmapsto M(x)
$$

for any given $x \in I$ has a right adjoint $F_{x *}: \operatorname{Mod}-R(x) \rightarrow \mathrm{Q} \operatorname{coh}(R)$ given by

$$
\left(F_{x *}(X)\right)(y)=X \otimes_{R(x)} R(x \vee y) \quad \text { for every } y \in I .
$$

These are the analogs of the inverse and the direct image functors in geometry; see [33, (7.8)]. Clearly, $F_{x *}$ sends flat $R(x)$-modules to flat quasi-coherent $R$ modules. For any $(p+1)$-tuple $i_{0}<\cdots<i_{p}$ of integers between 0 and $n$ we denote 
$M_{i_{0}, \ldots, i_{p}}=F_{x *} F_{x}^{*}(M)$ where $x=x_{i_{0}} \vee \cdots \vee x_{i_{p}}$. In particular we have the equality $M_{i_{0}, \ldots, i_{p}}(z)=M\left(z \vee x_{i_{0}} \vee \cdots \vee x_{i_{p}}\right)$ for each $z \in I$.

Next we construct for each $M \in \mathrm{Q} \operatorname{coh}(R)$ a so-called Čech resolution; see [37, $\S I I I .4$, p. 218]. This is a sequence of morphisms

$$
0 \longrightarrow M \stackrel{\partial}{\longrightarrow} \mathcal{C}^{0} M \stackrel{\partial}{\longrightarrow} \mathcal{C}^{1} M \stackrel{\partial}{\longrightarrow} \cdots \stackrel{\partial}{\longrightarrow} \mathcal{C}^{n} M \longrightarrow 0,
$$

where $\mathcal{C}^{p} M=\bigoplus_{i_{0}<i_{1}<\cdots<i_{p}} M_{i_{0}, i_{1}, \ldots i_{p}}$ for every $0 \leq p \leq n$. In order to describe the maps $\partial$, we need some more notation. Given $i_{0}<i_{1}<\cdots<i_{p}$, we shall denote by $\alpha_{i_{0}, i_{1}, \cdots, i_{p}}$ an element of $M_{i_{0}, i_{1}, \ldots, i_{p}}$. Then $\partial: \mathcal{C}^{p} M \rightarrow \mathcal{C}^{p+1} M$ will be given by the rule

$$
\partial(z):\left(\alpha_{i_{0}, i_{1}, \cdots, i_{p}}\right)_{i_{0}<i_{1}<\cdots<i_{p}} \longmapsto\left(\sum_{k=0}^{p+1}(-1)^{k} \alpha_{j_{0}, \ldots, \hat{j}_{k}, \ldots, j_{p+1}}\right)_{j_{0}<j_{1}<\cdots<j_{p+1}}
$$

for all $z \in I$, where $\hat{j}_{k}$ means "omit $j_{k}$." Strictly speaking we should have written $M_{y}^{x}\left(\alpha_{j_{0}, \ldots, \hat{j}_{k}, \ldots, j_{p+1}}\right)$ for suitable $x, y \in I$ on the right hand side, but we omit $M_{y}^{x}$ for the sake of clarity of the formula as there is no risk of confusion. It is rather easy to check that $(\Delta)$ is a complex in $\mathrm{Q} \operatorname{coh}(R)$.

More is true, however - we claim that $(\Delta)$ is an exact sequence. This is proved similarly to [37, Lemma III.4.2]. To this end we shall use the same convention as in [37, Remark 4.0.1]: if $i_{1}, \ldots, i_{p} \in\{0,1, \ldots, n\}$ is an arbitrary $(p+1)$-tuple, then $\alpha_{i_{0}, i_{1}, \cdots, i_{p}}$ will stand either for 0 if there is a repeated index, or for $\operatorname{sgn}(\sigma)$. $\alpha_{\sigma i_{0}, \sigma i_{1}, \cdots, \sigma i_{p}}$, where $\sigma$ is the permutation such that $\sigma i_{0}<\sigma i_{1}<\cdots<\sigma i_{p}$. Using this notation, observe that, for any fixed $z \in I$, the complex of $R(z)$-modules

$$
0 \longrightarrow M(z) \longrightarrow \mathcal{C}^{0} M(z) \longrightarrow \mathcal{C}^{1} M(z) \longrightarrow \cdots \longrightarrow \mathcal{C}^{n} M(z) \longrightarrow 0
$$

is contractible, where the null-homotopy $s: \mathcal{C}^{p} M(z) \rightarrow \mathcal{C}^{p-1} M(z)$ is given by the rule

$$
s:\left(\alpha_{i_{0}, i_{1}, \cdots, i_{p}}\right)_{i_{0}<i_{1}<\cdots<i_{p}} \longmapsto\left(\alpha_{z, j_{0}, j_{1}, \cdots, j_{p}}\right)_{j_{0}<j_{1}<\cdots<j_{p-1}}
$$

This proves the claim.

From this point we continue exactly as in [56, Proposition 3.19]. The cotorsion pair (Flat- $R(z)$, Cot- $R(z)$ ) is complete for each $z \in I$ by the above discussion. Using the approximation sequences, we construct a so-called proper flat resolution

$$
\cdots \longrightarrow P_{2}^{z} \longrightarrow P_{1}^{z} \longrightarrow P_{0}^{z} \longrightarrow M(z) \longrightarrow 0
$$

of $M(z)$ in Mod- $R(z)$. This by definition means that all $P_{i}^{z}$ are flat, the sequence is exact, and it remains exact when we apply the functor $\operatorname{Hom}_{R(z)}\left(P^{\prime},-\right)$ for any $P^{\prime} \in$ Flat- $R(z)$. Using the adjunction $\left(F_{z}^{*}, F_{z *}\right)$, it is easy to see that

$$
\cdots \longrightarrow F_{z *}\left(P_{2}^{z}\right) \longrightarrow F_{z *}\left(P_{1}^{z}\right) \longrightarrow F_{z *}\left(P_{0}^{z}\right) \longrightarrow F_{z *} F_{z}^{*}(M) \longrightarrow 0
$$

is a proper flat resolution in $\mathrm{Q} \operatorname{coh}(R)$. Summing over all $z=x_{i_{0}} \vee \cdots \vee x_{i_{p}}$ where $i_{0}<i_{1}<\cdots<i_{p}$, we obtain a proper flat resolution for each $\mathcal{C}^{p} M$ with $0 \leq p \leq n$. Finally, the argument described in detail in the proof of [56, Proposition 3.19] allows us to combine these to a proper flat resolution of $M$. 
For more special flat representations $R: I \rightarrow$ CommRings we may obtain left hand side classes of complete hereditary cotorsion pairs which are even closer to projective objects. A variety of such candidates and their appropriateness in geometry has been studied in [22, 23]. Here we mention only the simplest of them.

Definition 8.17. Let $R: I \rightarrow$ CommRings be a flat representation. A module $M \in \mathrm{Q} \operatorname{coh}(R)$ is called locally projective (or vector bundle in [13, §2]) if $M(i)$ is a projective $R(i)$-module for every $i \in I$. We will denote the class of locally projective quasi-coherent sheaves by Vect- $R$.

Proposition 8.18. Let $R: I \rightarrow$ CommRings be such that $\mathrm{Q} \operatorname{coh}(R) \stackrel{\cong}{\longrightarrow} \mathrm{Qcoh}(X)$ for a quasi-projective scheme $X$ over an affine scheme (see e.g. Examples 2.13 and 2.14). Then $\mathrm{Qcoh}(R)$ has a locally projective generator. In particular, we obtain a functorially complete hereditary cotorsion pair (Vect-R, Vect- $R^{\perp}$ ).

Proof. The first part follows from 69, Lemma 2.1.3]. The deconstructibility of Vect- $R$ is obtained by Proposition 8.13 and the rest is similar as for flat modules.

Remark 8.19. Interestingly enough, nothing seems to be known about the class Vect- $R^{\perp}$, it even seems to lack a name so far.

8.5. Monoidal models for derived categories of quasi-coherent sheaves. We conclude by summarizing our findings regarding the existence of monoidal model structures for $\mathbf{D}(\mathrm{Q} \operatorname{coh}(R))$.

Theorem 8.20. Let $R: I \rightarrow$ CommRings be a continuous flat representation of a finite upper semilattice $I$ (in particular for any quasi-compact separated scheme $X$ there is such an $R$ with $\mathrm{Q} \operatorname{coh}(R) \stackrel{\cong}{\longrightarrow} \mathrm{Qcoh}(X))$. Then there is a hereditary monoidal model structure on $\left(\mathbf{C}(\mathrm{Q} \operatorname{coh}(R)), \otimes, S^{0}(R), \mathcal{H o m}\right)$ (see Definitions 6.19 and 8.9) such that

(1) An object is cofibrant if and only if it is a retract of an $\left\{S^{n}(F) \mid F \in\right.$ Flat- $R$ and $n \in \mathbb{Z}\}$-filtered object.

(2) The class of trivial objects equals $\mathcal{W}=\mathbf{C}_{\mathrm{ac}}(\mathrm{Q} \operatorname{coh}(R))$.

(3) The class of fibrant objects equals $\tilde{\mathcal{A}}^{\perp}$, where we use Notation 7.6 for $\mathcal{A}=$ Flat- $R$.

If, moreover, $\mathrm{Q} \operatorname{coh}(R) \stackrel{\cong}{\longrightarrow} \operatorname{Qcoh}(X)$ for a quasi-projective scheme $X$ over an affine scheme, then there is another hereditary monoidal model structure on the category $\mathbf{C}(\mathrm{Qcoh}(R))$ such that

(1') An object is cofibrant if and only if it is a retract of an $\left\{S^{n}(V) \mid V \in\right.$ Vect- $R$ and $n \in \mathbb{Z}\}$-filtered object.

(2') The class of trivial objects equals $\mathcal{W}=\mathbf{C}_{\mathrm{ac}}(\mathrm{Q} \operatorname{coh}(R))$. 
(3') The class of fibrant objects equals $\left(\tilde{\mathcal{A}}^{\prime}\right)^{\perp}$, where we use Notation 7.6 for $\mathcal{A}^{\prime}=$ Vect $-R$.

In both cases, $\mathrm{Ho} \mathbf{C}(\mathrm{Q} \operatorname{coh}(R))$ is equal to $\mathbf{D}(\mathrm{Q} \operatorname{coh}(R))$ and the model structures are cofibrantly generated.

Proof. In order to construct the hereditary model structures on $\mathbf{C}(\mathrm{Q} \operatorname{coh}(R))$, we simply input the two complete hereditary cotorsion pairs (Flat- $R, \operatorname{Cot}-R$ ) and (Vect- $R$, Vect- $R^{\perp}$ ) to Theorem 7.16 . The fact that the model structures are cofibrantly generated follows, in view of Remark 17.15, from the results in 88.4. To prove that the model structures are monoidal, it remains to notice that assumption (3) from Theorem 8.11 is satisfied whenever every cofibrant complex has all components flat. This follows by analyzing the monoidal structure on $\mathbf{C}(\mathrm{Qcoh}(R))$ as described in 88.1 and boils down to the following well known fact: If a short exact sequence $\varepsilon: 0 \rightarrow X \rightarrow Y \rightarrow Z \rightarrow 0$ of right modules over a ring $T$ has the last term $Z$ flat, then $\varepsilon \otimes_{T} U$ is exact for any left $T$-module $U$.

\section{References}

[1] J. Adámek, H. Herrlich, J. Rosický, and W. Tholen. Weak factorization systems and topological functors. Appl. Categ. Structures, 10(3):237-249, 2002. Papers in honour of the seventieth birthday of Professor Heinrich Kleisli (Fribourg, 2000).

[2] J. Adámek and J. Rosický. Locally presentable and accessible categories, volume 189 of London Mathematical Society Lecture Note Series. Cambridge University Press, Cambridge, 1994.

[3] L. Alonso Tarrío, A. Jeremías López, and J. Lipman. Local homology and cohomology on schemes. Ann. Sci. École Norm. Sup. (4), 30(1):1-39, 1997.

[4] L. Angeleri Hügel, D. Happel, and H. Krause, editors. Handbook of tilting theory, volume 332 of London Mathematical Society Lecture Note Series. Cambridge University Press, Cambridge, 2007.

[5] G. Azumaya and A. Facchini. Rings of pure global dimension zero and Mittag-Leffler modules. J. Pure Appl. Algebra, 62(2):109-122, 1989.

[6] H. Becker. Models for singularity categories. Preprint, arXiv:1205.4473v1, 2012.

[7] A. Beligiannis and I. Reiten. Homological and homotopical aspects of torsion theories. Mem. Amer. Math. Soc., 188(883):viii+207, 2007.

[8] L. Bican, R. El Bashir, and E. Enochs. All modules have flat covers. Bull. London Math. Soc., 33(4):385-390, 2001.

[9] M. Bökstedt and A. Neeman. Homotopy limits in triangulated categories. Compositio Math., 86(2):209-234, 1993.

[10] T. Bühler. Exact categories. Expo. Math., 28(1):1-69, 2010.

[11] C. Casacuberta and A. Neeman. Brown representability does not come for free. Math. Res. Lett., 16(1):1-5, 2009.

[12] W. Crawley-Boevey. Locally finitely presented additive categories. Comm. Algebra, 22(5):1641-1674, 1994. 
[13] V. Drinfeld. Infinite-dimensional vector bundles in algebraic geometry: an introduction. In The unity of mathematics, volume 244 of Progr. Math., pages 263-304. Birkhäuser Boston, Boston, MA, 2006.

[14] P. C. Eklof and A. H. Mekler. Almost free modules, volume 65 of North-Holland Mathematical Library. North-Holland Publishing Co., Amsterdam, revised edition, 2002. Set-theoretic methods.

[15] P. C. Eklof and J. Trlifaj. How to make Ext vanish. Bull. London Math. Soc., 33(1):41-51, 2001.

[16] R. El Bashir. Covers and directed colimits. Algebr. Represent. Theory, 9(5):423-430, 2006.

[17] E. Enochs and S. Estrada. Relative homological algebra in the category of quasicoherent sheaves. Adv. Math., 194(2):284-295, 2005.

[18] E. Enochs, S. Estrada, J. R. García Rozas, and L. Oyonarte. Flat covers in the category of quasi-coherent sheaves over the projective line. Comm. Algebra, 32(4):14971508, 2004.

[19] E. Enochs and L. Oyonarte. Flat covers and cotorsion envelopes of sheaves. Proc. Amer. Math. Soc., 130(5):1285-1292 (electronic), 2002.

[20] E. E. Enochs. Shortening filtrations. Sci. China Math., 55(4):687-693, 2012.

[21] E. E. Enochs and O. M. G. Jenda. Relative homological algebra. Volume 2, volume 54 of de Gruyter Expositions in Mathematics. Walter de Gruyter GmbH \& Co. KG, Berlin, 2011.

[22] S. Estrada, P. Guil Asensio, and J. Trlifaj. Descent of restricted flat Mittag-Leffler modules and generalized vector bundles. To appear in Proc. Amer. Math. Soc., arXiv:1110.5364v1, 2011.

[23] S. Estrada, P. A. Guil Asensio, M. Prest, and J. Trlifaj. Model category structures arising from Drinfeld vector bundles. Adv. Math., 231(3-4):1417-1438, 2012.

[24] P. Freyd. Abelian categories. An introduction to the theory of functors. Harper's Series in Modern Mathematics. Harper \& Row Publishers, New York, 1964.

[25] P. Gabriel and F. Ulmer. Lokal präsentierbare Kategorien. Lecture Notes in Mathematics, Vol. 221. Springer-Verlag, Berlin, 1971.

[26] P. Gabriel and M. Zisman. Calculus of fractions and homotopy theory. Ergebnisse der Mathematik und ihrer Grenzgebiete, Band 35. Springer-Verlag New York, Inc., New York, 1967.

[27] J. Gillespie. The flat model structure on $\mathrm{Ch}(R)$. Trans. Amer. Math. Soc., 356(8):3369-3390 (electronic), 2004.

[28] J. Gillespie. The flat model structure on complexes of sheaves. Trans. Amer. Math. Soc., 358(7):2855-2874 (electronic), 2006.

[29] J. Gillespie. Kaplansky classes and derived categories. Math. Z., 257(4):811-843, 2007.

[30] J. Gillespie. Cotorsion pairs and degreewise homological model structures. Homology, Homotopy Appl., 10(1):283-304, 2008.

[31] J. Gillespie. Model structures on exact categories. J. Pure Appl. Algebra, 215(12):2892-2902, 2011. 
[32] R. Göbel and J. Trlifaj. Approximations and endomorphism algebras of modules, volume 41 of de Gruyter Expositions in Mathematics. Walter de Gruyter GmbH \& Co. KG, Berlin, 2006.

[33] U. Görtz and T. Wedhorn. Algebraic geometry I. Advanced Lectures in Mathematics. Vieweg + Teubner, Wiesbaden, 2010. Schemes with examples and exercises.

[34] A. Grothendieck. Sur quelques points d'algèbre homologique. Tôhoku Math. J. (2), 9:119-221, 1957.

[35] D. Happel. Triangulated categories in the representation theory of finite-dimensional algebras, volume 119 of London Mathematical Society Lecture Note Series. Cambridge University Press, Cambridge, 1988.

[36] R. Hartshorne. Residues and duality. Lecture notes of a seminar on the work of A. Grothendieck, given at Harvard 1963/64. With an appendix by P. Deligne. Lecture Notes in Mathematics, No. 20. Springer-Verlag, Berlin, 1966.

[37] R. Hartshorne. Algebraic geometry. Springer-Verlag, New York, 1977. Graduate Texts in Mathematics, No. 52.

[38] P. S. Hirschhorn. Model categories and their localizations, volume 99 of Mathematical Surveys and Monographs. American Mathematical Society, Providence, RI, 2003.

[39] H. Holm. Gorenstein homological algebra. PhD thesis, University of Copenhagen, 2004. Available at http://www.math.ku.dk/ holm/download/PhDthesis.pdf

[40] H. Holm and P. Jørgensen. Covers, precovers, and purity. Illinois J. Math., 52(2):691-703, 2008.

[41] M. Hovey. Model categories, volume 63 of Mathematical Surveys and Monographs. American Mathematical Society, Providence, RI, 1999.

[42] M. Hovey. Cotorsion pairs, model category structures, and representation theory. Math. Z., 241(3):553-592, 2002.

[43] M. Hovey. Cotorsion pairs and model categories. In Interactions between homotopy theory and algebra, volume 436 of Contemp. Math., pages 277-296. Amer. Math. Soc., Providence, RI, 2007.

[44] T. Jech. Set theory. Springer Monographs in Mathematics. Springer-Verlag, Berlin, 2003. The third millennium edition, revised and expanded.

[45] P. Jørgensen. The homotopy category of complexes of projective modules. Adv. Math., 193(1):223-232, 2005.

[46] B. Keller. Chain complexes and stable categories. Manuscripta Math., 67(4):379-417, 1990.

[47] B. Keller. Deriving DG categories. Ann. Sci. École Norm. Sup. (4), 27(1):63-102, 1994.

[48] H. Krause. Derived categories, resolutions, and Brown representability. In Interactions between homotopy theory and algebra, volume 436 of Contemp. Math., pages 101-139. Amer. Math. Soc., Providence, RI, 2007.

[49] J. Lurie. Higher topos theory, volume 170 of Annals of Mathematics Studies. Princeton University Press, Princeton, NJ, 2009.

[50] S. Mac Lane. Homology. Die Grundlehren der mathematischen Wissenschaften, Bd. 114. Academic Press Inc., Publishers, New York, 1963. 
[51] S. Mac Lane. Categories for the working mathematician, volume 5 of Graduate Texts in Mathematics. Springer-Verlag, New York, second edition, 1998.

[52] M. Makkai. Rearranging colimits: A categorical lemma due to Jacob Lurie. Available at http://www.math.mcgill.ca/makkai/Various/Binder1.pdf, 2008.

[53] G. Maltsiniotis. Le théorème de Quillen, d'adjonction des foncteurs dérivés, revisité. C. R. Math. Acad. Sci. Paris, 344(9):549-552, 2007.

[54] H. Matsumura. Commutative ring theory, volume 8 of Cambridge Studies in Advanced Mathematics. Cambridge University Press, Cambridge, second edition, 1989. Translated from the Japanese by M. Reid.

[55] B. Mitchell. The full imbedding theorem. Amer. J. Math., 86:619-637, 1964.

[56] D. Murfet. The Mock Homotopy Category of Projectives and Grothendieck Duality. PhD thesis, Australian National University, 2007. Available at http://www.therisingsea.org/thesis.pdf

[57] A. Neeman. The homotopy category of flat modules, and Grothendieck duality. Invent. Math., 174(2):255-308, 2008.

[58] A. Neeman. Dualizing complexes - the modern way. In Cycles, motives and Shimura varieties, Tata Inst. Fund. Res. Stud. Math., pages 419-447. Tata Inst. Fund. Res., Mumbai, 2010.

[59] A. Neeman. Some adjoints in homotopy categories. Ann. of Math. (2), 171(3):21432155, 2010.

[60] D. G. Quillen. Homotopical algebra. Lecture Notes in Mathematics, No. 43. SpringerVerlag, Berlin, 1967.

[61] M. Raynaud and L. Gruson. Critères de platitude et de projectivité. Techniques de "platification" d'un module. Invent. Math., 13:1-89, 1971.

[62] J. Rosický. Flat covers and factorizations. J. Algebra, 253(1):1-13, 2002.

[63] W. Rump. Flat covers in abelian and in non-abelian categories. Adv. Math., 225(3):1589-1615, 2010.

[64] L. Salce. Cotorsion theories for abelian groups. In Symposia Mathematica, Vol. XXIII (Conf. Abelian Groups and their Relationship to the Theory of Modules, INDAM, Rome, 1977), pages 11-32. Academic Press, London, 1979.

[65] M. Saorín and J. Štovíček. On exact categories and applications to triangulated adjoints and model structures. Adv. Math., 228(2):968-1007, 2011.

[66] B. Stenström. Rings of quotients. Springer-Verlag, New York, 1975. Die Grundlehren der Mathematischen Wissenschaften, Band 217, An introduction to methods of ring theory.

[67] J. Štovíček. Deconstructibility and Hill lemma in Grothendieck categories. To appear in Forum Math., doi:10.1515/FORM.2011.113, preprint at arXiv:1005.3251v2, 2010.

[68] J. Št'ovíček and D. Pospíšil. On compactly generated torsion pairs and the classification of co-t-structures for commutative noetherian rings. Preprint, arXiv:1212.3122v1, 2012.

[69] R. W. Thomason and T. Trobaugh. Higher algebraic $K$-theory of schemes and of derived categories. In The Grothendieck Festschrift, Vol. III, volume 88 of Progr. Math., pages 247-435. Birkhäuser Boston, Boston, MA, 1990. 
[70] R. Wisbauer. Grundlagen der Modul- und Ringtheorie. Verlag Reinhard Fischer, Munich, 1988. Ein Handbuch für Studium und Forschung. [A handbook for study and research].

[71] G. Yang and Z. Liu. Cotorsion pairs and model structures on $\mathrm{Ch}(R)$. Proc. Edinb. Math. Soc. (2), 54(3):783-797, 2011.

Jan Šţovíček, Department of Algebra, Faculty of Mathematics and Physics, Charles University in Prague, Sokolovská 83, 18675 Praha 8, Czech Republic

E-mail: stovicek@karlin.mff.cuni.cz 